\title{
An Empirical Test of Gain-Loss Separability in Prospect Theory*
}

\author{
George $\mathrm{Wu}^{\dagger} \quad$ Alex B. Markle
}

November 26, 2007

\begin{abstract}
We investigate a basic premise of prospect theory, that the valuation of gains and losses is separable. In prospect theory, gain-loss separability implies that a mixed gamble is valued by summing the valuations of the gain and loss portions of that gamble. Two experimental studies demonstrate a systematic violation of the double matching axiom, an axiom that is necessary for gain-loss separability. We document a reversal between preferences for mixed gambles and the associated gain and loss gambles - mixed gamble $A$ is preferred to mixed gamble $B$, but the gain and loss portions of $B$ are preferred to the gain and loss portions of $A$. The observed choice patterns are consistent with a process in which individuals are less sensitive to probability differences when choosing among mixed gambles than when choosing among either gain or loss gambles.
\end{abstract}

Key Words Risky choice, prospect theory, mixed gambles, double matching, probability weighting function.

\footnotetext{
${ }^{*}$ We thank Manel Baucells, Han Bleichrodt, David Budescu, Philippe Delquie, John Payne, Suzanne Shu, Horst Zank, and Jiao Zhang for their comments and help with this paper. We give the usual special thanks to Peter Wakker for his thorough and insightful comments. We also thank Jim Smith for his guidance in shaping this research. The authors are also grateful to attendees of the 2004 FUR conference, the 2004 JDM conference, and the 2004 BDRM conference for their helpful suggestions.

${ }^{\dagger}$ Center for Decision Research, University of Chicago, Graduate School of Business, Chicago, IL 60637 (wu@ChicagoGSB.edu)

${ }^{\ddagger}$ New York University, Stern School of Business, New York, NY 10012 (amarkle@stern.nyu.edu)
} 


\section{Introduction}

Prospect theory distinguishes itself from the classical theory of decision under risk, expected utility theory, in taking change in wealth rather than absolute wealth to be the relevant carrier of value (Kahneman and Tversky, 1979; Tversky and Kahneman, 1992). This distinction has been applied with enormous success to applications in business, finance, law, medicine, and political science (e.g., Barberis, Huang, and Santos, 2001; Camerer, 2000; Jolls, Sunstein, and Thaler, 1998; McNeil et al., 1982; Quattrone and Tversky, 1988). Indeed, most important real world decisions are mixed gambles, involving some possibility of gain and some possibility of loss (MacCrimmon and Wehrung, 1990; March and Shapira, 1987).

This article investigates how individuals choose among mixed gambles by examining a fundamental assumption of prospect theory, gain-loss separability. Simply stated, gain-loss separability requires that preferences for gains be independent of preferences for losses and, more strongly, that the valuation of a mixed gamble be the sum of the valuations of the gain and loss portions of that gamble. A failure of gain-loss separability has practical as well as theoretical implications. First, empirical findings gleaned from the numerous studies of single-domain gambles will not necessarily generalize to the domain of mixed gambles. Second, procedures to elicit loss aversion coefficients usually assume separability between gains and losses and thus may require modification (e.g., Abdellaoui, Bleichrodt and Paraschiv, 2007; Schmidt and Traub, 2002). Finally, models that relax gain-loss separability will necessarily be more complex than models that assume separability.

We examine gain-loss separability by testing an axiom known as double matching, a necessary condition for gain-loss separability within prospect theory (Tversky and Kahneman, 1992). We define double matching formally in the next section, but, before doing so, we motivate and illustrate our primary test of double matching with an example. We gave participants a choice between the following two gambles:

$\underline{\text { Problem } 1(n=81)}$

$$
H=\left(\begin{array}{c}
.50 \text { chance at } \$ 4200 \\
.50 \text { chance at } \$-3000
\end{array}\right) \text { vs. } L=\left(\begin{array}{c}
.75 \text { chance at } \$ 3000 \\
.25 \text { chance at } \$-4500
\end{array}\right)
$$


Levy and Levy (2002a) ran a variant of this choice problem in which the gain in $H$ was $\$ 4500$ (Experiment 1, Test I). In our study, a slight majority of participants preferred $H$ to $L$ (the percentage of participants choosing each option is given in the square brackets). Gain-loss separability requires that mixed gamble $H$ be preferred to mixed gamble $L$ if the gain portion of $H$ is preferred to the gain portion of $L$ and the loss portion of $H$ is preferred to the loss portion of $L$. In the next section, we show that this idea is precisely what is captured by the double matching axiom.

To test gain-loss separability, we decomposed gambles $H$ and $L$ into gain gambles, $H^{+}$and $L^{+}$, and loss gambles, $H^{-}$and $L^{-}$. The same participants were also asked to choose between $H^{+}$and $L^{+}$and between $H^{-}$and $L^{-}$. The gambles and choice percentages are shown below:

Problem $2(n=81)$

$$
H^{+}=\left(\begin{array}{c}
.50 \text { chance at } \$ 4200 \\
.50 \text { chance at } \$ 0
\end{array}\right) \text { vs. } L^{+}=\left(\begin{array}{c}
.75 \text { chance at } \$ 3000 \\
.25 \text { chance at } \$ 0
\end{array}\right)
$$

Problem $3(n=81)$

$$
H^{-}=\left(\begin{array}{c}
.50 \text { chance at } \$ 0 \\
.50 \text { chance at } \$-3000
\end{array}\right) \text { vs. } L^{-}=\left(\begin{array}{c}
.75 \text { chance at } \$ 0 \\
.25 \text { chance at } \$-4500
\end{array}\right)
$$

A majority of participants preferred the gain portion of $L$ to the gain portion of $H$, as well as the loss portion of $L$ to the loss portion of $H$. Nonetheless, a slight majority of participants chose mixed gamble $H$ to mixed gamble $L$, thus violating double matching.

More generally, we hypothesize that individuals are less sensitive to differences in probabilities when choosing between mixed gambles (Problem 1) than when faced with a choice between gain or loss gambles (Problems 2 or 3). Indeed, if individuals are completely insensitive to probabilities when faced with Problem 1, $H$ will appear to dominate $L$, since the best outcome in $H(+4200)$ is higher than the best outcome in $L(+3000)$, and the worst outcome in $H(-3000)$ is higher than the worst outcome in $L(-4500)$.

The remainder of the paper presents a more detailed empirical investigation of gain-loss separability and demonstrates that the violation of gain-loss separability illustrated above is not unique. 
In Section 2, we review prospect theory, present a formal definition of double matching, and review relevant empirical findings. Section 3 presents our primary empirical test of double matching. This investigation consists of over 30 variants of the problem presented in the Introduction, the vast majority showing the same pattern of violations of gain-loss separability. We also formalize an error model analysis and show that our choice patterns are systematic and not due to random error. In addition, estimates of the probability weighting function to the choice data support our psychological interpretation that individuals are less sensitive to probability differences when making choices among mixed gambles than when faced with gambles involving all gains or all losses. In Section 4, we establish the robustness of our double matching violations by employing a gamble configuration that differs from the problem presented above. Section 5 concludes with a discussion of the implications of this research, potential psychological drivers of our results, and some directions for future research.

\section{Prospect Theory and Gain-Loss Separability}

\subsection{Prospect Theory Basics}

Both versions of prospect theory, original prospect theory (Kahneman and Tversky, 1979) and cumulative prospect theory (Tversky and Kahneman, 1992), assume gain-loss separability. We present cumulative prospect theory here because that theory applies to gambles with an arbitrary number of outcomes.

We denote a gamble $P=\left(x_{1}, p_{1} ; \ldots ; x_{k}, p_{k} ; 0, p_{0} ; x_{k+1}, p_{k+1} ; \ldots ; x_{n}, p_{n}\right)$, where $p_{i}$ indicates the probability of outcome $x_{i}$. Outcomes are rank-ordered, $x_{1}>\ldots>x_{k}>0>x_{k+1}>\ldots>x_{n}$, where $x_{1}, \ldots, x_{k}$ are gains and $x_{k+1}, \ldots, x_{n}$ are losses relative to a reference point or neutral outcome 0. A gamble is a mixed gamble if there is a strictly positive probability of a gain and a strictly positive probability of a loss, i.e., $\sum_{i=1}^{k} p_{i}>0$ and $\sum_{i=k+1}^{n} p_{i}>0$.

A mixed gamble can be parsed into a gain gamble,

$$
P^{+}=\left(x_{1}, p_{1} ; \ldots ; x_{k}, p_{k} ; 0, p_{0}+\sum_{i=k+1}^{n} p_{i}\right)
$$


and a loss gamble,

$$
P^{-}=\left(0, \sum_{i=1}^{k} p_{i}+p_{0} ; x_{k+1}, p_{k+1} ; \ldots ; x_{n}, p_{n}\right)
$$

such that the probability of a loss (gain) is moved to the neutral outcome 0 so that probabilities for any gain (loss) gamble sum to one. A single-domain gamble is either a gain gamble or a loss gamble. Preferences for gambles are denoted in the standard fashion: $P \succeq(\succ) Q$ denotes that $P$ is weakly (strictly) preferred to $Q . P$ is indifferent to $Q$ (denoted $P \sim Q$ ) if $P \succeq Q$ and $Q \succeq P$.

In cumulative prospect theory, the utility of a mixed gamble is simply the sum of the utilities of the gain and loss gambles:

$$
U(P)=U\left(P^{+}\right)+U\left(P^{-}\right)
$$

(2.1) represents preferences in the sense that $P \succeq Q$ if and only if

$$
U(P)=U\left(P^{+}\right)+U\left(P^{-}\right) \geq U(Q)=U\left(Q^{+}\right)+U\left(Q^{-}\right) .
$$

A necessary condition for the separability of gains and losses in (2.2) is the double matching axiom (Tversky and Kahneman, 1992, p. 318).

Double Matching For all mixed gambles, $P$ and $Q$, if $P^{+} \sim Q^{+}$and $P^{-} \sim Q^{-}$, then $P \sim Q$.

The necessity of double matching for the cumulative prospect theory representation follows immediately from (2.2). Wakker and Tversky (1993, p. 157) invoke a slightly weaker axiom called gain-loss consistency and show that this axiom is usually implied by other axioms and thus can often be dropped.

$P^{+}$and $P^{-}$are valued as follows:

$$
U\left(P^{+}\right)=\pi^{+}\left(p_{1}\right) v\left(x_{1}\right)+\sum_{i=2}^{k}\left[\pi^{+}\left(\sum_{j=1}^{i} p_{j}\right)-\pi^{+}\left(\sum_{j=1}^{i-1} p_{j}\right)\right] v\left(x_{i}\right)
$$


and

$$
U\left(P^{-}\right)=\pi^{-}\left(p_{n}\right) v\left(x_{n}\right)+\sum_{i=k+1}^{n-1}\left[\pi^{-}\left(\sum_{j=i}^{n} p_{j}\right)-\pi^{-}\left(\sum_{j=i+1}^{n} p_{j}\right)\right] v\left(x_{i}\right)
$$

where $v(\cdot)$ is a value function, and $\pi^{+}(\cdot)$ is the probability weighting function for gains and $\pi^{-}(\cdot)$ is the probability weighting function for losses. In addition, the value function is scaled such that $v(0)=0$ and exhibits loss aversion if $-v(-x)>v(x)$ for all $x .{ }^{1}$ Both $\pi^{+}(\cdot)$ and $\pi^{-}(\cdot)$ are non-decreasing with $\pi^{+}(0)=\pi^{-}(0)=0$ and $\pi^{+}(1)=\pi^{-}(1)=1$. In (2.3) and (2.4), the value of each outcome is multiplied by a decision weight that typically differs from the probability of that outcome. The representation is called rank-dependent because the decision weight attached to an outcome depends on the rank of that outcome (Quiggin, 1982). The representation for losses in (2.4) "mirrors" that for gains in the sense that the weighting function begins with the most extreme outcomes first (the worst loss in (2.4) and the best gain in (2.3)) and then works toward the reference point.

\subsection{Empirical Tests of Mixed Gambles}

A large number of studies have highlighted the descriptive shortcomings of expected utility theory (for reviews, see Camerer 1995; Fox and See, 2003; Luce, 2000; Machina, 1987; Starmer 2000; Wu, Zhang, and Gonzalez, 2004). Prospect theory can explain most of these empirical findings, although Birnbaum and his colleagues have documented a series of patterns that cannot be accommodated by cumulative prospect theory (e.g., Birnbaum, 2004; Birnbaum and McIntosh, 1996; Birnbaum and Navarette, 1998).

Tversky and Kahneman (1992) conducted the first comprehensive investigation of the shape of the value and probability weighting functions. They found that the value function was concave for gains, convex for losses, and exhibited loss aversion, and that the probability weighting function was inverse S-shaped (first concave, then convex) for both gains and losses, with the weighting function for losses slightly less curved and more elevated than the weighting function for gains. Many subsequent studies have replicated these basic results using both choice and matching tasks

\footnotetext{
${ }^{1}$ See Abdellaoui, Bleichrodt, and Paraschiv (2007) for other definitions of loss aversion.
} 
(e.g., Abdellaoui, 2000; Bleichrodt and Pinto, 2000; Camerer and Ho, 1994; Etchart-Vincent, 2004; Fehr-Duda, de Gennaro, and Schubert, 2006; Gonzalez and Wu, 1999; Lattimore, Baker, and Witte, 1992; Tversky and Fox, 1995; Wu and Gonzalez, 1996).

A variety of empirical studies have involved mixed gambles. However, most of these studies were not explicitly designed to compare choices between mixed gambles and single-domain gambles, but instead to test the expected utility model (e.g., Mosteller and Nogee, 1951; Davidson, Suppes, and Siegel, 1957), or to measure loss aversion (e.g., Abdellaoui, Bleichrodt and Paraschiv, 2007; Brooks and Zank, 2005; Schmidt and Traub, 2002; see also Köbberling and Wakker, 2005). Another set of studies used duplex gambles, which involve the joint receipt of a simple gain gamble and a simple loss gamble, to test whether decision makers used certain information processing strategies (e.g., Payne and Braunstein, 1971; Slovic and Lichtenstein, 1968). Slovic (1967) postulated an axiom called duplex decomposition (see also Luce, 2000) that requires that a mixed gamble be indifferent to the joint receipt of the gain and loss portions of the mixed gamble. Preliminary empirical studies have been mixed about the validity of duplex decomposition (e.g., Cho, Troung, and Haneda, 2005).

A smaller number of studies have investigated direct properties of mixed gambles. Payne, Laughhunn, and Crum (1980) showed a reversal of preference depending on whether a mixed gamble was translated by adding or subtracting a constant amount from each outcome, a finding consistent with loss aversion (see, also, Payne, Laughhunn, and Crum, 1981). Chechile and Butler (2000, 2003) tested whether mixed gambles could be described by a bilinear form, of which prospect theory is a special case. Participants were given one mixed gamble, and were asked to provide a probability of winning for a second mixed gamble so that they were indifferent between the two gambles. While these data appear to be inconsistent with a bilinear form, the analytical procedure used in these investigations is quite complex and relies on parametric assumptions about the form of the value function and the probability weighting function. Payne (2005) documented choice patterns for mixed gambles that are inconsistent with cumulative prospect theory assuming typical parameter values. Payne argued that these patterns demonstrate that individuals sometimes use a heuristic of selecting the mixed gamble with the highest probability of a gain.

Levy and Levy (2002a,b) presented several empirical patterns that they argued contradicted prospect theory's S-shaped value function and instead supported an inverse S-shaped value func- 
tion, convex for gains and concave for losses, as originally proposed by Markowitz (1952). A variant of one of the patterns was presented in the introduction. Wakker (2003) and Baucells and Heukamp (2004) critiqued their analysis and showed that these patterns are consistent with prospect theory if the probability weighting function is sufficiently inverse S-shaped (see, also, Baucells and Heukamp, 2006). Baltussen, Post, and van Vliet (2006) studied a variant of Levy and Levy's (2002a) Experiment 2. Their data are consistent with the functional form of cumulative prospect theory, but requires a probability weighting function that is much more linear for gains than observed in other studies and more curved for losses than for gains, contrary to the previously reviewed studies.

\section{Study 1}

In this section, we present the results of the first of two empirical investigations of gain-loss separability. We employed a within-participants test of the double matching axiom using variants of the example presented in the introduction. The empirical test used a choice between "simple" mixed gambles with one gain and one loss outcome, $H=(g, p ; l, 1-p)$ and $L=\left(g^{\prime}, p^{\prime} ; l^{\prime}, 1-p^{\prime}\right)$, where $g>g^{\prime}>0$ and $0>l>l^{\prime} . H$ and $L$ are mnemonic for "High" and "Low" in the sense that $H$ has higher outcomes than $L$, a higher gain $\left(g>g^{\prime}\right)$ and a less severe loss $\left(l>l^{\prime}\right)$.

To test double matching, we parsed $H$ and $L$ into gain gambles and loss gambles and asked the same participants to choose between $H^{+}$and $L^{+}$, as well as $H^{-}$and $L^{-}$. Choice patterns of $H \succ L, L^{+} \succ H^{+}$, and $L^{-} \succ H^{-}\left(H L^{+} L^{-}\right.$, for short $)$or $L \succ H, H^{+} \succ L^{+}$, and $H^{-} \succ L^{-}$ $\left(L H^{+} H^{-}\right.$, for short) violate double matching.

\subsection{Method}

We recruited 453 students from The University of Chicago to participate in this study. The study consisted of 6 separate surveys. Each survey contained 4 to 7 tests of double matching (12 to 21 questions) as well as several filler questions to bring the total number of questions to approximately 20 to 25 binary choices. The first 3 surveys were administered in the form of survey booklets. For each survey, 6 booklets were created in which we randomized the order of the choices and counterbalanced the location of the gambles in each pair (left or right side of sheet). The final 


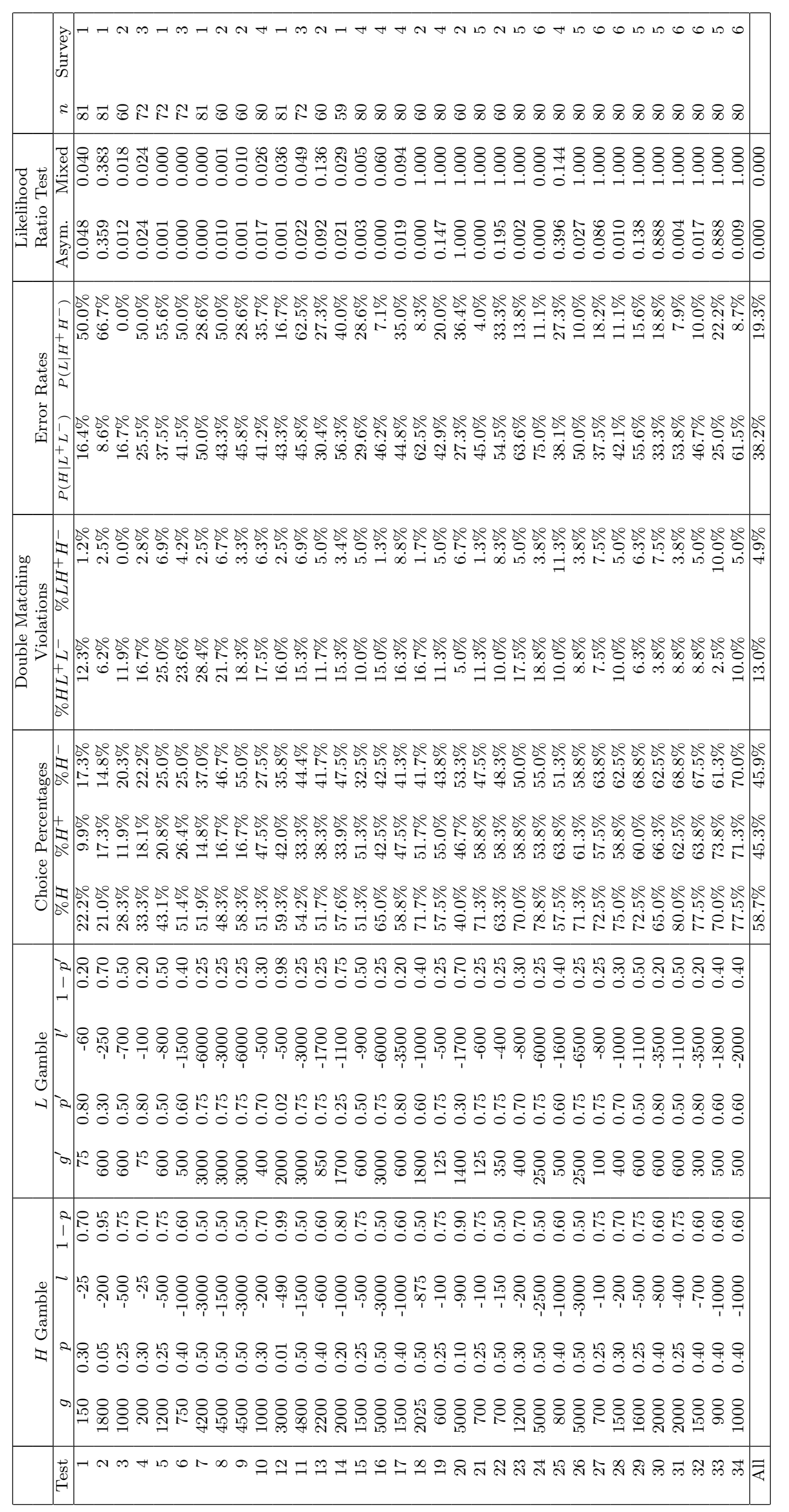

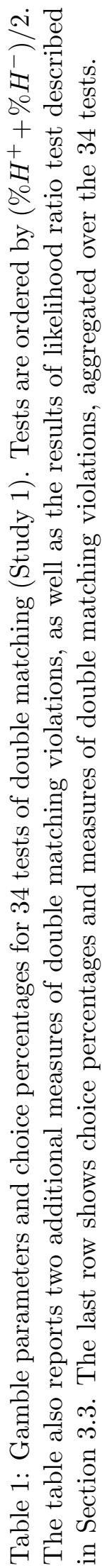


3 surveys were run on computer, with the order of choices randomized and location of gambles counterbalanced for each participant. ${ }^{2}$ Participants were paid $\$ 3$ to $\$ 4$ for completing the survey booklet.

All choices were hypothetical. There were two major reasons for not using monetary incentives. First and foremost, mixed gambles involve the possibility of loss. Using gambles with real losses imposes obvious practical and methodological limitations, including the necessity of limiting the stakes of the gambles and the probability of loss (e.g., Thaler and Johnson, 1990, p. 653). Second, the conclusions drawn from hypothetical choice studies do not appear to be qualitatively different from those in which participants were paid based on their choices (Camerer, 1989; Camerer and Hogarth, 1999), although participants were generally more risk-averse for real choices than for hypothetical choices.

The entire empirical study of double matching involved 34 tests (listed in Table 1). We varied the gambles in several respects to test the robustness of gain-loss separability. The expected value was sometimes identical for both options, but generally was not. The probability of a gain varied substantially across our mixed gambles, ranging from .01 to .80. Hence, the probability of a loss varied from .20 to .99 . We also varied the relative attractiveness of $H^{+}$and $L^{+}$, and $H^{-}$and $L^{-}$, to create tests in which the majority of participants would choose $L^{+}$over $H^{+}$and $L^{-}$over $H^{-}$, and others in which the majority of participants would prefer $\mathrm{H}^{+}$and $\mathrm{H}^{-}$. To do so, some of the tests were variants of others (e.g., Tests 7, 9, 16, 24, and 26), in which the outcome values were varied to change the relative attractiveness of $H^{+}$and $H^{-3}$

\subsection{Summary Results}

Choice models are generally formulated in terms of deterministic axioms, but statistical tests should reflect the possibility that decision makers make errors when deciding (Luce and Suppes, 1965). Thus, the statistical testing of binary choice data like ours poses notable challenges (Iverson and Falmagne, 1985; Myung, Karabatsos, and Iverson, 2005). We deal with these difficulties by first

\footnotetext{
${ }^{2}$ In a pre-test of our computer program, we replicated 12 of the questions used in the original 3 survey booklets. The correlation between the choice percentages for the booklet and computer was .96 .

${ }^{3}$ Test 7 was presented in the introduction. The mixed gambles (but not the gain or loss gambles) in Tests 8,9 , and 18 were studied by Levy and Levy $(2002 \mathrm{a}, \mathrm{b})$.
} 
presenting four measures of double matching violations, each of which provides a slightly different view of the data. We take these measures to be suggestive, as it is possible that some of these results could be produced by random error. In Section 3.3, we develop several error models to determine whether the pattern of data are systematic or caused by random error. This analysis also provides a statistical test for our data and shows that random error cannot account for the full pattern of results.

Choice percentages for all gambles are found in Table $1 .{ }^{4}$ We let $\% H$ denote the percentage of participants who chose mixed gamble $H$ over mixed gamble $L$. Similarly, $\% H^{+}$and $\% H^{-}$denote the percentage of participants who chose $\mathrm{H}^{+}$over $\mathrm{L}^{+}$, and $\mathrm{H}^{-}$over $\mathrm{L}^{-}$, respectively. Earlier, we suggested that the violation of double matching in our introductory example was consistent with a process in which participants were less sensitive to probability differences for mixed gambles than for gain gambles or loss gambles. Such a process favors mixed gamble $H$ over mixed gamble $L$, since $H$ has a higher upside as well as a lower downside. Indeed, this process is consistent with the empirical pattern, $\% H>\max \left(\% H^{+}, \% H^{-}\right)$, observed in 29 of the 34 tests (sign test: $\left.p<.0001\right)$.

Three alternative measures examine how mixed gamble $H$ is "advantaged" in choice relative to mixed gamble $L$. First, we look at the relative proportion of the two types of double matching violations, $H L^{+} L^{-}$and $L H^{+} H^{-}$(with $\% H L^{+} L^{-}$and $\% L^{+} H^{-}$denoting the respective percentages of each violation). In our introductory example (Test 7), 30.9\% of participants violated double matching, with $28.4 \%$ choosing $H, L^{+}$, and $L^{-}$and only $2.5 \%$ choosing $L, H^{+}$, and $H^{-}$. Table 1 contains double matching violation rates for each of the 34 tests. Overall, 28 of the 34 tests showed the same pattern: $\% H L^{+} L^{-}>\% L H^{+} H^{-}$(sign test: $p<.0001$ ). Across all 34 tests, $H L^{+} L^{-}$violations occurred 2.7 times more often than $L H^{+} H^{-}$violations $\left(\% H L^{+} L^{-}=13.0 \%\right.$ and $\left.\% \mathrm{LH}^{+} \mathrm{H}^{-}=4.9 \%\right)$.

Next, we consider the relationship between two conditional probabilities, $P\left(H \mid L^{+} L^{-}\right)$and $P\left(L \mid H^{+} H^{-}\right)$. The pattern $\% H L^{+} L^{-}>\% L H^{+} H^{-}$could reflect a disproportionate percentage of participants preferring $L^{+}$and $L^{-}$relative to $H^{+}$and $H^{-}$. However, we find, consistent with our hypothesized process, that $P\left(H \mid L^{+} L^{-}\right)>P\left(L \mid H^{+} H^{-}\right)$for 26 of the 34 tests (sign test: $p<.01$ ).

\footnotetext{
${ }^{4}$ The e-companion (http://mansci.journal.informs.org/) contains choice percentages for all 8 possible choice patterns, $\mathrm{HH}^{+} \mathrm{H}^{-}, \mathrm{HH}^{+} \mathrm{L}^{-}$, etc.
} 
Moreover, across all tests, $P\left(H \mid L^{+} L^{-}\right)=38.2 \%$ is almost twice as large as $P\left(L \mid H^{+} H^{-}\right)=19.3 \%$. These conditional probabilities are also reported in Table 1.

Finally, we examine the likelihood of choosing $H$ over $L$ for the two "indeterminate" choice patterns where double matching does not apply: $H^{+} L^{-}$and $L^{+} H^{-}$. Our hypothesized story suggests that $H$ is advantaged relative to $L$, or $P\left(H \mid H^{+} L^{-}\right)>.5$ and $P\left(H \mid L^{+} H^{-}\right)>.5$, while the null hypothesis requires that the two conditional probabilities equal .5. These conditional probabilities show the same pattern as the other measures: $P\left(H \mid H^{+} L^{-}\right)>.5$ for 26 of the 34 tests (sign test: $p<.001$ ), while $P\left(H \mid L^{+} H^{-}\right)>.5$ for 25 of the 34 tests (sign test: $p<.01$ ). Aggregated over the 34 tests, $P\left(H \mid H^{+} L^{-}\right)=63.4 \%$ and $P\left(H \mid L^{+} H^{-}\right)=61.2 \%$. We report $P\left(H \mid H^{+} L^{-}\right)$and $P\left(H \mid L^{+} H^{-}\right)$for each of the 34 tests in the e-companion.

\subsection{Statistical Tests}

Could our violations of double matching be a statistical artifact generated by random error? Random error models have been proposed to explain expected utility violations (Ballinger and Wilcox 1997; Hey, 1995; Hey and Orme 1994), overconfidence (Brenner 2000; Erev, Wallsten, and Budescu, 1994), and better-than-average effects (Burson, Larrick, and Klayman, 2006; Krueger and Mueller, 2002). For example, the introductory example might be explained by a "regression effect": if $\% H^{+}$ and $\% H^{-}$are close to 0 , then $\% H$ will generally be closer to .5 , and hence larger than either $\% H^{+}$ or $\% H^{-}$.

To rule out random error explanations for our violations of double matching, we compare a random error model in which the error rate for choosing among gambles is identical for gain, loss, and mixed gambles (the null error model) with a systematic error model motivated by our hypothesized process that individuals are less sensitive to probability differences when choosing among mixed gambles than when choosing among single-domain gambles (the asymmetric error model). We also consider a second asymmetric error model in which the error rate for choosing among mixed gambles is higher than the error rate for choosing among gain or loss gambles (the mixed error model). The error analysis provides a formal statistical test for the data in Study 1 and shows that the asymmetric error model provides a significantly better account of the double matching violations than either the null or mixed error models. In addition, the analysis shows 
that whereas the mixed error model is consistent with some of our double matching violations, it cannot account for the full empirical pattern of data. ${ }^{5}$

The three proposed error models consist of assumptions about preference types, satisfaction of the double matching axiom, and error rates. These assumptions are described below.

We first make an assumption about preference types. We assume that there are four types, $\theta_{H^{+} H^{-}}, \theta_{H^{+} L^{-}}, \theta_{L^{+} H^{-}}$, and $\theta_{L^{+} L^{-}}$. Each type has "underlying preferences," though their "revealed preferences" reflect some error (see below). Type $\theta_{H^{+} H^{-}}$prefers $H^{+}$over $L^{+}$and $H^{-}$over $L^{-}$, type $\theta_{H^{+} L^{-}}$prefers $H^{+}$over $L^{+}$and $L^{-}$over $H^{-}$, etc.

We next assume that "underlying preferences" satisfy double matching. Type $\theta_{H^{+} H^{-}}$prefers $H^{+}$over $L^{+}$and $H^{-}$over $L^{-}$, and thus $H$ over $L$, whereas type $\theta_{L^{+} L^{-}}$prefer $L^{+}$over $H^{+}$and $L^{-}$ over $H^{-}$, and hence $L$ over $H$. We consider the other two types, $\theta_{H^{+} L^{-}}$and $\theta_{L^{+} H^{-}}$, "indeterminate" and assume that they choose according to their gain or loss preferences with equal probability.

Finally, we assume that decision makers choose with error. Let $\epsilon_{S}$ be the error rate for singledomain gambles, such that $P\left(L^{+} \succ H^{+} \mid \theta_{H^{+} H^{-}}\right)=P\left(L^{-} \succ H^{-} \mid \theta_{H^{+} H^{-}}\right)=P\left(H^{+} \succ L^{+} \mid \theta_{L^{+} L^{-}}\right)=$ $P\left(H^{-} \succ L^{-} \mid \theta_{L^{+} L^{-}}\right)=\epsilon_{S}$. The remaining conditional probabilities are defined analogously.

The three proposed error models differ in their treatment of the choice between mixed gambles $H$ and $L$. Let $\epsilon_{L}=P\left(L \succ H \mid \theta_{H^{+} H^{-}}\right)$and $\epsilon_{H}=P\left(H \succ L \mid \theta_{L^{+} L^{-}}\right)$. The three error models below make different restrictions on the relationship among the three error rates, $\epsilon_{S}, \epsilon_{L}, \epsilon_{H}$ :

- "Null" model: $\epsilon_{S}=\epsilon_{L}=\epsilon_{H}$;

- "Mixed" error model: $\epsilon_{L}=\epsilon_{H}>\epsilon_{S}$;

- "Asymmetric" error model: $\epsilon_{H}>\epsilon_{S}=\epsilon_{L}$.

The null model assumes that the same error rate applies to all gambles, whereas the mixed model assumes that a higher error rate applies to mixed gambles than single-domain gambles, perhaps because mixed gambles are more complicated than single-domain gambles. The asymmetric model

\footnotetext{
${ }^{5}$ In the e-companion, we also derive implications of each of the three error models for the four measures of double matching violations discussed in Section 3.2. This analysis shows that the mixed error model is consistent with the observed double matching violations when $\left(\% H^{+}+\% H^{-}\right) / 2$ is less than $50 \%$, but not when this average exceeds $50 \%$.
} 
allows a different error rate for type $\theta_{L^{+} L^{-}}$than type $\theta_{H^{+} H^{-}}$. The empirical pattern, $\% H>$ $\max \left(\% H^{+}, \% H^{-}\right)$, as well as the hypothesized process suggests that $\epsilon_{H}>\epsilon_{L}=\epsilon_{S} \cdot{ }^{6}$

To test the significance of each double matching violation, we employ a likelihood ratio test, which performed a similar role in Tversky $(1969,1972)$ and Wu, Zhang, and Abdellaoui (2005). We first describe the details of the procedure and then provide the results of the statistical test.

Let $\mathbf{p}$ denote the vector of probabilities of the underlying types. Furthermore, let $L_{0}\left(D ; \mathbf{p}, \epsilon_{S}\right)$ be the likelihood of the data $D$ under the null model for a given set of type parameters $\mathbf{p}$ and error rate, $\epsilon_{S}$, with $L_{0}^{*}(D)=\max _{\mathbf{p}, \epsilon_{S}} L_{0}\left(D \mid \mathbf{p}, \epsilon_{S}\right)$ denoting the maximum likelihood for the null model. We also conduct maximum likelihood estimations on the mixed and asymmetric error models and use a likelihood ratio test to determine whether the extra parameters used in those models provides a significantly better fit. For example, let $L_{A}\left(D ; \mathbf{p}, \epsilon_{S}, \epsilon_{H}\right)$ be the likelihood of the data under the asymmetric model, with $L_{A}^{*}(D)=\max _{\mathbf{p}, \epsilon_{S}, \epsilon_{H}} L_{A}\left(D \mid \mathbf{p}, \epsilon_{S}, \epsilon_{H}\right)$ the maximum likelihood for the asymmetric model. Then the statistic $2 \ln \left[L_{A}^{*}(D) / L_{0}^{*}(D)\right]$ is distributed approximately $\chi^{2}(1)$ (e.g., Mood and Graybill, 1963). In all cases, $0 \leq \epsilon_{S}, \epsilon_{H}, \epsilon_{L} \leq \frac{1}{2}$.

We fit the choice patterns for each of the 34 double matching tests using this procedure. Table 1 contains a comparison of the mixed and asymmetric error models relative to the null error model. This analysis shows that the asymmetric model provides a significantly better account of our choice data than either the null or mixed error models. The asymmetric model shows a significantly better fit than the null model in $71 \%$ of the tests (using the conventional $p<.05$ standard). In contrast, the mixed model fits better than the null model in only $41 \%$ of the tests (again using $p<.05$ ). Note that the asymmetric and mixed model perform similarly when $\left(\% H^{+}+\% H^{-}\right) / 2<.5$. However, when $\left(\% H^{+}+\% H^{-}\right) / 2>.5$, only 1 of 14 tests are significant with the mixed model, while 8 of 14 tests are significant with the asymmetric model. The e-companion contains a more complete reporting of the likelihood ratio test, include a table of the estimated error rates, as well as a detailed discussion of the direction of the estimated error rates.

\footnotetext{
${ }^{6}$ Since the two indeterminate types, $\theta_{H^{+} L^{-}}$and $\theta_{L^{+} H^{-}}$, choose like type $\theta_{H^{+} H^{-}}$or type $\theta_{L^{+} L^{-}}$with equal probability, $P\left(H \succ L \mid \theta_{H^{+} L^{-}}\right)=P\left(H \succ L \mid \theta_{L^{+} H^{-}}\right)=(1 / 2) P\left(H \succ L \mid \theta_{H^{+} H^{-}}\right)+(1 / 2) P\left(H \succ L \mid \theta_{L^{+} L^{-}}\right)=\frac{1}{2}\left(1-\epsilon_{L}+\right.$ $\left.\epsilon_{H}\right)$. For the asymmetric model, $\epsilon_{H}>\epsilon_{L}$, and thus $P\left(H \succ L \mid \theta_{H^{+} L^{-}}\right)>\frac{1}{2}$.
} 


\subsection{Probability Weighting Function Estimation}

We formally test the hypothesis that violations of double matching result from a diminished sensitivity to probability differences for mixed gambles relative to gain or loss gambles by fitting a probability weighting function to our double matching test data. We use a probability weighting function specification proposed by Tversky and Kahneman (1992), and used by used by Abdellaoui (2000), Camerer and Ho (1994), and Wu and Gonzalez (1996):

$$
\pi(p)=\frac{p^{\gamma}}{\left(p^{\gamma}+(1-p)^{\gamma}\right)^{1 / \gamma}}
$$

The parameter $\gamma$ captures the degree of curvature and elevation. There is no probability distortion if $\gamma=1$, and more pronounced curvature and lower elevation with smaller values of $\gamma$.

To test the hypothesis that the probability weighting function is more curved for mixed gambles than gain or loss gambles, we let $\gamma$ differ for single-domain gambles (gains or losses) $\left(\gamma_{S}\right)$ and mixed gambles $\left(\gamma_{M}\right)$. Our hypothesis requires that $\gamma_{M}<\gamma_{S}$.

Since our data are binary, we employ a stochastic choice analysis akin to Baucells and Heukamp (2004), Camerer and Ho (1994), and Wu and Gonzalez (1996). This "representative-agent" approach assumes that all respondents have the same underlying preferences but make errors in their choices. For a discussion of the assumptions behind this method, see McFadden (1981).

Let $P(A \succ B)$ be the probability of choosing option $A$ over option $B$. We assume that this probability is a function of the utility of option $A, U(A)$, and the utility of option $B, U(B)$ : $P(A \succ B)=f(U(A), U(B))$ (see Luce and Suppes, 1965). We use a logistic function to describe this probabilistic relation,

$$
P(A \succ B)=\frac{1}{1+\exp (-\mu(U(A)-U(B))}
$$

The scaling parameter $\mu$ captures the sensitivity of choices to utility differences. If $\mu=0$, all choices are decided by a coin toss (i.e., $P(A \succ B)=.5$ for all $A$ and $B$ ). As $\mu \rightarrow \infty, P(A \succ B) \rightarrow 1$ when $U(A)>U(B)$, and $P(A \succ B) \rightarrow 0$ when $U(A)>U(B)$.

We assume that the utility of a gamble is determined by cumulative prospect theory (Eqns. 
(2.1), (2.3)-(2.4)). We follow Tversky and Kahneman (1992) in using a power function specification for the value function,

$$
v(x)= \begin{cases}x^{\alpha_{G}}, & x>0 \\ -\lambda(-x)^{\alpha_{L}}, & \text { otherwise }\end{cases}
$$

The power function is invariant to multiplicative scaling and becomes more concave for gains as $\alpha_{G}$ decreases and more convex for losses as $\alpha_{L}$ decreases. The loss aversion coefficient, $\lambda$, captures the extent to which losses loom larger than gains.

Our stochastic choice specification chooses the best fitting $\gamma_{S}$ and $\gamma_{M}$, where the objective function is to maximize the likelihood of the data. Let $j=1, \ldots, 34$ indicate a test of double matching, with $n_{j}$ denoting the number of participants for test $j$, and $\% H_{j}, \% H_{j}^{+}$, and $\% H_{j}^{-}$, denoting the percentage of participants who chose $H_{j}$ over $L_{j}, H_{j}^{+}$over $L_{j}^{+}$, and $H_{j}^{-}$over $L_{j}^{-}$, respectively. Then the objective function is:

$$
\begin{aligned}
\operatorname{Max}_{\mu, \gamma_{S}, \gamma_{M}} & \prod_{j=1}^{34} P\left(H_{j} \succ L_{j}\right)^{\left(n_{j}\right)\left(\% H_{j}\right)}\left(1-P\left(H_{j} \succ L_{j}\right)\right)^{\left(n_{j}\right)\left(1-\% H_{j}\right)} \times \\
& P\left(H_{j}^{+} \succ L_{j}^{+}\right)^{\left(n_{j}\right)\left(\% H_{j}^{+}\right)}\left(1-P\left(H_{j}^{+} \succ L_{j}^{+}\right)\right)^{\left(n_{j}\right)\left(1-\% H_{j}^{+}\right)} \times \\
& P\left(H_{j}^{-} \succ L_{j}^{-}\right)^{\left(n_{j}\right)\left(\% H_{j}^{-}\right)}\left(1-P\left(H_{j}^{-} \succ L_{j}^{-}\right)\right)^{\left(n_{j}\right)\left(1-\% H_{j}^{-}\right)} .
\end{aligned}
$$

Thus, the likelihood is maximized over the 7593 choices in Table 1. The optimization problem was implemented using a nonlinear optimization procedure written in MATLAB, with $\mu, \gamma_{S}$, and $\gamma_{M}$ left as free parameters.

We conducted a variety of analyses to test our hypothesis. We held $\alpha_{G}, \alpha_{L}$, and $\lambda$ constant in our analyses, first setting $\lambda=2$ and $\alpha_{G}=\alpha_{L}=.5$ and then performing sensitivity analysis on these parameters to test the robustness of the results. We fixed these parameters at these levels in our base analysis for several reasons. First, $\alpha$ and $\gamma$ cannot be identified uniquely with the simple gambles used in our study (see, e.g., Prelec, 1998). Second, Abdellaoui (2000) and Tversky and Kahneman (1992) estimated power function coefficients that were nearly identical for gains and losses. Third, we used a power function coefficient of .5 because this estimate was produced by Wu 
and Gonzalez (1996) using a similar procedure. Fourth, the loss aversion coefficient reflects the parameter estimates of Tversky and Kahneman (1992), as well as the endowment effect studies of Kahneman, Knetsch, and Thaler (1990).

The estimation results support our hypothesis that the weighting function is more curved for mixed gambles than single-domain gambles, thereby lending support to our hypothesis that participants are less sensitive to intermediate probabilities with mixed gambles than with single-domain gambles. Our weighting function estimate for single-domain gambles, $\hat{\gamma}_{S}$, was .67, close to the parameter estimate of .71 from Wu and Gonzalez (1996). In contrast, the parameter value for mixed gambles was considerably lower, $\hat{\gamma}_{M}=.55$, a difference that was statistically significant $(t=620.9, p<.0001)$. (The best-fitting scaling parameter was found to be $\hat{\mu}=.18$.) These estimates are plotted in Figure 1. We conducted a variety of sensitivity analyses to test whether this finding was robust and found that $\hat{\gamma}_{M}$ was significantly lower than $\hat{\gamma}_{S}$ for all combinations of values of $\alpha$ from .3 to 1.0 and $\lambda$ from 1 to 2.5 (see e-companion for details).

To further investigate the robustness of our analysis, we replaced the form in Eq. (3.2) with the weighting function proposed by Prelec (1998), $\pi(p)=\exp \left(-(-\ln p)^{\beta}\right)$. The Prelec weighting function is the identity function when $\beta=1$, approaches a step function as $\beta \rightarrow 0$, and has a fixed point at $1 / e \approx .368$ (i.e., $\pi(1 / e)=1 / e$ ). We found qualitatively identical results $\left(\hat{\beta}_{S}=.61\right.$, $\left.\hat{\beta}_{M}=.47, t=589.6, p<.0001\right)$, with the Prelec function performing slightly worse in terms of $\log$ likelihood.

We also estimated a separate set of models in which we allowed $\gamma$ in the Tversky and Kahneman weighting function to differ for gain gambles, loss gambles, and mixed gambles. Specifically, we let $\gamma_{G S}, \gamma_{L S}, \gamma_{G M}=\gamma_{G S}-\delta$, and $\gamma_{L M}=\gamma_{L S}-\delta$ be the parameters for gain gambles, loss gambles, the gain portions of mixed gambles, and the loss portions of mixed gambles, respectively. Thus, $\delta$ captures the difference in curvature between mixed gambles and single-domain gambles. This specification yielded similar results to the analyses presented earlier. Contrary to most previous studies, the parameter for losses, $\hat{\gamma}_{L S}=.66$, was substantially lower than for gains, $\hat{\gamma}_{G S}=.76$ (however, see Baltussen et al., 2006), but most critically, the parameter capturing the difference between single-domain gambles and mixed gambles, $\hat{\delta}=.14$ was significantly positive $(t=587.9$, $p<.0001$ ), indicating a more curved weighting function for mixed gambles than for single-domain 
gambles. We also produced similar results assuming the weighting function proposed by Prelec (1998).

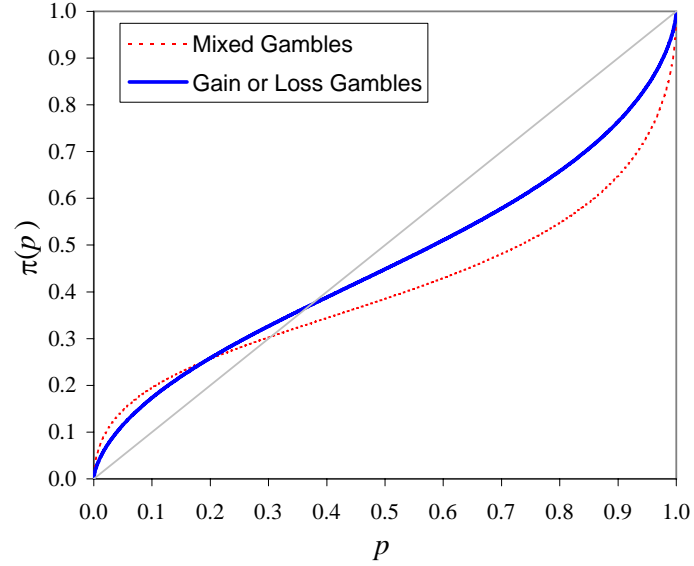

Study 1

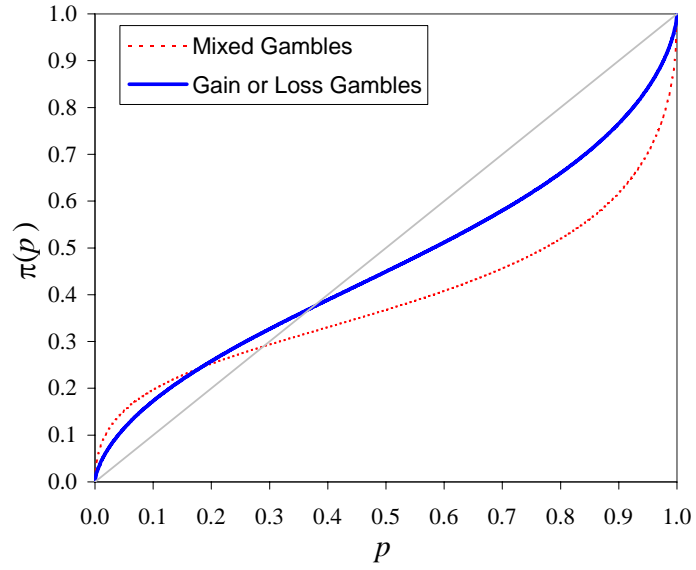

Study 2

Figure 1: Probability weighting functions, $\pi(p)$, for mixed gambles and single-domain gambles, estimated from Study 1 and Study 2 choice data using a stochastic choice functional and the Tversky and Kahneman (1992) function.

\section{$4 \quad$ Study 2}

We suggested at the end of Section 3.4 that the violations of gain-loss separability in Study 1 were due to a diminished sensitivity to probabilities for mixed gambles than single-domain gambles. However, all the gambles in Study 1 have a particular configuration - the gain and loss outcomes in the $H$ gambles are larger than the respective gain and loss outcomes in the $L$ gamble. Thus, an alternative explanation for our observed violations of gain-loss separability is that participants may be using an "extreme outcome heuristic." This heuristic, while interesting, clearly has less generality than our explanation in terms of diminished sensitivity to probabilities. Study 2 addresses this confound by introducing an indirect choice test of gain-loss separability.

Consider the following sets of gambles,

$$
H^{+}=\left(\begin{array}{c}
p \text { chance at } \$ x \\
1-p \text { chance at } \$ 0
\end{array}\right) \text { vs. } L^{+}=\left(\begin{array}{c}
q \text { chance at } \$ y \\
1-q \text { chance at } \$ 0
\end{array}\right) \text {, }
$$

and 


$$
\left[H^{+}, C^{-}\right]=\left(\begin{array}{c}
p \text { chance at } \$ x \\
1-p-r \text { chance at } \$ 0 \\
r \text { chance at } \$-z
\end{array}\right) \text { vs. }\left[L^{+}, C^{-}\right]=\left(\begin{array}{c}
q \text { chance at } \$ y \\
1-q-r \text { chance at } \$ 0 \\
r \text { chance at } \$-z
\end{array}\right)
$$

where $x>y$ and $p<q . H^{+}$and $L^{+}$are gain gambles, with the mixed gambles, $\left[H^{+}, C^{-}\right]$ and $\left[L^{+}, C^{-}\right]$, created by concatenating the loss gamble, $C^{-}=(1-r, 0 ; r,-z)$, to $H^{+}$and $L^{+}$, respectively. A violation of gain-loss separability occurs if $H^{+} \succ($ resp. $\prec) L^{+}$but $\left[L^{+}, C^{-}\right] \succ$ (resp. $\prec)\left[H^{+}, C^{-}\right]$. If decision makers are more sensitive to probability differences when choosing among single-domain gambles than mixed gambles as in Study 1, then the relative preference for $q$ chance at $\$ y$ over $p$ chance at $\$ x$ is greatest when the choice involves two gain gambles than when it involves two mixed gambles.

Of course, direct choice between these gambles confounds our diminished sensitivity to probabilities story with the extreme outcome heuristic. In addition, the choice between $\left[H^{+}, C^{-}\right]$and $\left[L^{+}, C^{-}\right]$may promote cancellation or editing of common outcomes (e.g., Kahneman and Tversky, 1979; Wu, 1994). To circumvent these concerns, we compare $H^{+}$and $L^{+}$each with one alternative, $A^{+}$, and $\left[H^{+}, C^{-}\right]$and $\left[L^{+}, C^{-}\right]$separately with a second alternative $B$. The second alternative $B$ could be a mixed gamble, a sure thing gain or loss, or a gain or loss gamble. This indirect test thus involves a comparison of the choice percentages for four pairs of gambles, $P\left(H^{+}, A^{+}\right), P\left(L^{+}, A^{+}\right)$, $P\left(\left[H^{+}, C^{-}\right], B\right)$, and $P\left(\left[L^{+}, C^{-}\right], B\right)$. Note that the design of this study has an advantage and disadvantage relative to the design used in Study 1. The indirect test uses a different configuration of gambles than Study 1 and hence investigates the generality of our violations of gain-loss separability. On the other hand, the test is indirect and more complicated than the test used in Study 1. Thus any violations of gain-loss separability will be less transparent than those observed in our previous study. ${ }^{7}$

\subsection{Methods}

We recruited 102 students from the University of Chicago to participate in this study. As in the previous study, we employed a within-participants design. Participants made binary choices

\footnotetext{
${ }^{7}$ Since the loss portion of the gambles coincide, this design also tests sign-comonotonic independence (see Wakker and Tversky, 1993).
} 
between 24 unique gamble pairs (see Table 4). The choices of $H^{+}$versus $A^{+}$and $L^{+}$versus $A^{+}$ were used in two separate tests (Tests 1 and 2, 3 and 4, etc.) and thus our design produced 8 total tests of gain-loss separability. The gambles were presented on a computer, with the order of the gambles randomized and the location of each gamble in the pair (left or right) counterbalanced for each participant. Participants were paid $\$ 3$ for completing the task. As with the previous study, all choices are hypothetical.

\subsection{Summary Results}

Choice percentages for Study 2 are shown in Table 2. In examining the results, we first present a broad overview of the results by examining choice percentages. Choice percentages are suggestive although imprecise. Thus, we subsequently develop a formal test of whether gain-loss separability is violated.

Gain-loss separability requires that adding the common loss $C^{-}$not change preferences between $H^{+}$and $L^{+}$, i.e., $H^{+} \succ($ resp. $\prec) L^{+}$if and only if $\left[H^{+}, C^{-}\right] \succ($ resp. $\prec)\left[L^{+}, C^{-}\right]$. Thus, $P\left(H^{+}, A^{+}\right)<P\left(L^{+}, A^{+}\right)$and $P\left(\left[H^{+}, C^{-}\right], B\right)>P\left(\left[L^{+}, C^{-}\right], B\right)$ constitutes a violation of gain-loss separability in the predicted direction. We observe exactly these patterns in tests 1 , 3, and 4 . Moreover, the second differences, $\left(P\left(H^{+}, A^{+}\right)-P\left(L^{+}, A^{+}\right)\right)-\left(P\left(\left[H^{+}, C^{-}\right], B\right)-P\left(\left[L^{+}, C^{-}\right], B\right)\right)$, are negative as predicted for 7 of the 8 tests and for the average of all tests.

\subsection{Statistical Tests}

These measures constitute weak and imprecise tests of double-matching: Violations can occur in the absence of choice percentage reversals, and it is unclear how to interpret second differences in choice percentages. To test gain-loss separability formally, we develop two stochastic choice models, one which constrains preferences to follow gain-loss separability and one which does not. We then test whether the unconstrained model provides a better fit to the choice data. We begin by making the simplifying but standard assumption that the probability that prospect $S$ is chosen over prospect $T$ is captured by the logit model, $P(S \succ T)=\frac{1}{1+\exp (-\mu(U(S)-U(T)))}$.

Under this assumption, fitting the choice percentages in Table 2 involves estimating 6 free parameters for each of the 8 tests: $U\left(H^{+}\right), U\left(L^{+}\right), U\left(\left[H^{+}, C^{-}\right]\right), U\left(\left[L^{+}, C^{-}\right]\right), U\left(A^{+}\right)$, and $U(B)$ 


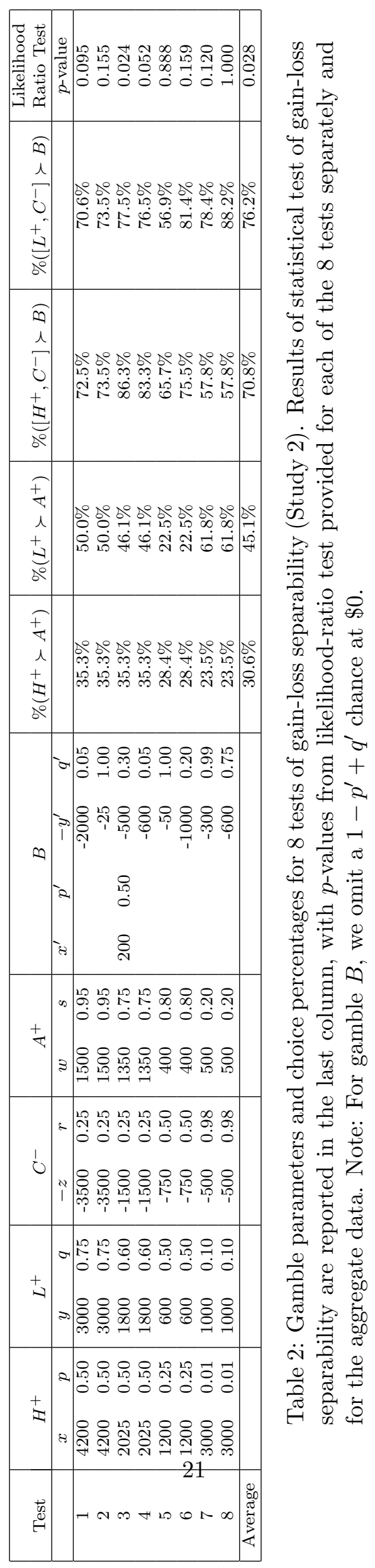


( since $U(\cdot)$ can be rescaled multiplicatively, we are free to set $\mu=1$ ). However, gain-loss separability imposes the restriction that $U\left(\left[H^{+}, C^{-}\right]\right)=U\left(H^{+}\right)+U\left(C^{-}\right)$and $U\left(\left[L^{+}, C^{-}\right]\right)=U\left(L^{+}\right)+U\left(C^{-}\right)$, leaving 5 free parameters: $U\left(H^{+}\right), U\left(L^{+}\right), U\left(C^{-}\right), U\left(A^{+}\right)$, and $U(B)$. To test whether gain-loss separability is violated, we thus test whether the unconstrained 6 parameter model provides a significantly better fit to the data than the 5 parameter model that imposes gain-loss separability.

We fit both models to the choice data using maximum likelihood estimation. (Additional details are found in the e-companion.) We first estimate each model separately for the 8 tests of gain-loss separability. We then use the likelihood-ratio test to determine whether the unconstrained model fits the data significantly better than the constrained model. The last column of Table 2 shows $p$-values for each of the 8 tests. The estimates for 6 of the 8 tests are in the hypothesized direction, with 1 of the 8 tests significant at the 0.05 level, and 2 others are marginally significant $(p<0.10)$. Of course, the 8 tests are not independent since some of the choices appear in two tests.

Finally, we estimate parameters for the choices of all 8 tests simultaneously. The unconstrained model yields a significantly better fit $(p<.03)$ than the constrained model, adjusting for the 4 additional parameters (one for each $U\left(C^{-}\right)$).

\subsection{Probability Weighting Function Estimation}

We followed the same procedure outlined in Section 3.4 to estimate probability weighting functions for mixed gambles $\left(\gamma_{M}\right)$ and single-domain gambles for the data from Study 2. Each of the 102 participants in Study 2 made 24 choices, thus the likelihood is maximized over the 2448 choices in Table 2. All other aspects of the procedure were identical.

As with Study 1, our base analysis assumed that $\alpha=\alpha_{G}=\alpha_{L}=0.5$ and $\lambda=2$. The resulting parameter estimates were very close to the Study 1 estimates: $\hat{\gamma}_{S}=.68$ and $\hat{\gamma}_{M}=.52$. More critically, the difference between $\hat{\gamma}_{S}$ and $\hat{\gamma}_{M}$ was statistically significant $(t=8.28, p<.0001)$. The best-fitting scaling parameter was $\hat{\mu}=.28$, somewhat higher than the estimate of Study 1 . The estimates for the Prelec weighting function were also remarkably close to those found for the Study

1 data: $\hat{\beta}_{S}=.64$ and $\hat{\beta}_{M}=.44(p<.0001)$. The estimates for the Tversky and Kahneman function are plotted in Figure 1.

We also conducted sensitivity analyses on $\alpha$ and $\lambda$ to test whether these differences were robust. 
The e-companion shows that $\hat{\gamma}_{S}>\hat{\gamma}_{M}$ for most values of $\alpha$ and $\lambda$. However, unlike Study $1, \hat{\gamma}_{S}<\hat{\gamma}_{M}$ when $\alpha \geq .9$. It is important to note, however, that the fits for $\alpha \geq .9$ were notably worse than the fits when $\alpha$ was low. ${ }^{8}$

\section{General Discussion}

Studies 1 and 2 demonstrated systematic violations of double matching, an axiom that is necessary for gain-loss separability. These violations are consistent with a process in which individuals are less sensitive to differences in probabilities when choosing among mixed gambles than when choosing among gambles with either all gains or all losses. Direct support for this interpretation is provided by our estimates of the probability weighting functions for Studies 1 and 2. In both studies, we found that the weighting function was indeed more curved for mixed gambles than for single-domain gambles.

Below, we offer two possible psychological explanations, one affective and one cognitive, for our violations of gain-loss separability. We conclude with some final remarks about the contribution of our paper.

\subsection{Affective Explanation}

Affect-based accounts have been evoked to explain a number of decision making phenomena (cf., Rottenstreich and Shu, 2004; Slovic et al., 2002). Rottenstreich and Hsee (2001) found that the probability weighting function was significantly more curved for lotteries over affect-rich objects (such as a hypothetical kiss from a favorite movie star or a hypothetical electric shock) than for lotteries over affect-poor objects (such as monetary gains). To the extent that losses are inherently affect-rich, there may be a "spill-over" to gains in which mixed gambles evoke an affective rather than a calculative mindset (Hsee and Rottenstreich, 2004). Such a mindset would lead to less sensitivity to intermediate probability differences for mixed gambles relative to single-domain gambles.

\footnotetext{
${ }^{8}$ To illustrate the difference in fit, we computed the mean absolute difference (MAD) between the actual choice percentages and the fitted choice percentages. The MAD for $\alpha=0.5$ and $\lambda=2$ was .096, compared to a MAD of .192 for $\alpha=0.9$ and $\lambda=2$.
} 


\subsection{Cognitive Explanation}

We suggest that mixed gambles are more complex psychologically than single-domain gambles. Losses are not merely the opposite of gains, but gains and losses appear to be processed in different parts of the brain (Bechara et al., 1997; Breiter et al., 2001), and seem to be distinct psychologically and not just two ends of a continuum (Larsen et al., 2004). As a result, rules that may be used to simplify single-domain gambles may not translate well to simplifying mixed gambles. For example, while decision makers may use an editing operation such as combination to simplify single-domain gambles (e.g., Tversky and Kahneman, 1979), such a heuristic is not readily applicable to mixed gambles, where the outcomes are "apples and oranges."

Decision makers may deal with the complexity introduced by mixed gambles by using constructive decision making processes to simplify the choice (e.g., Payne, Bettman, and Johnson, 1992). We discussed one heuristic that may be used for choosing among mixed gambles - the reduction of a mixed gamble to extreme outcomes, the best gain and the worst loss. Of course, whereas this heuristic can explain the results of Study 1, it does not account for the violations of double matching in Study 2. This heuristic might be even more appealing for mixed gambles with a large number of outcomes. Indeed, Birnbaum and Bahra (2007) produced violations of double matching with gambles that are more complex than the ones used in our investigation. These violations are consistent with an "extreme outcome heuristic." This account also recalls March and Shapira's (1987) finding that managers evaluate uncertain prospects in terms of the "worst or best (plausible) case" (p. 1411). ${ }^{9}$

To the extent that heuristics are used to simplify mixed gambles, other mixed gamble configurations may encourage the use of other heuristics. Indeed, Payne (2005) found that participants faced with a multiple-outcome mixed gamble preferred to improve the $\$ 0$ outcome rather than the best gain, contrary to some parametric specifications of cumulative prospect theory. These results suggest that, individuals may use a heuristic of maximizing the probability of gain, or minimizing the probability of loss, when faced with a complicated mixed gamble. It does not appear that our participants are using a heuristic of this sort to evaluate our gambles, since this heuristic would

\footnotetext{
${ }^{9}$ It is interesting to note that an extreme outcome heuristic will produce behavior that resembles the use of Hurwicz's (1951) "pessimism-optimism index criterion" of maximizing the weighted average of the maximum and the minimum for each option.
} 
generate a violation of double matching in the opposite direction of our empirical findings. However, this heuristic is surely applied in some choice situations. Process tracing methods such as Mouselab may help researchers understand when and why decision makers employ a probability heuristic or an extreme outcome heuristic (e.g., Payne, Bettman, and Johnson, 1992).

\subsection{Concluding Remarks}

In the last 50 years, a large body of empirical research has investigated how decision makers choose among risky gambles. Most of these findings can be accommodated by prospect theory. An Sshaped value function and inverse S-shaped probability weighting function can model the reflection effect, the fourfold pattern of risk preferences, the common-ratio and common-consequence effects, as well as the generalization of these findings from risk to uncertainty. However, the majority of the existing empirical evidence has involved single-domain gambles. The emphasis on these gambles is sensible - they are easy for research participants to understand and can be studied in hypothetical situations as well as played out for real payoffs.

The study of single-domain gambles is justified if the understanding gleaned from these investigations extends to the domain of mixed gambles. Our study indicates that mixed gamble behavior is described well by an S-shaped value function and an inverse S-shaped probability weighting function. However, gain-loss separability fails, and hence different parameter values are needed for mixed gambles than single-domain gambles. As a result, findings inferred from studies of singledomain gambles may not extend automatically to mixed gambles.

Our violations of gain-loss separability appear to be systematic. Nevertheless, we do not regard our results as providing a general picture of how decision makers choose among mixed gambles. The mixed gambles in Study 1 have a very special configuration that may contribute to a "perceived" dominance: the highest outcome in $H$ is better than the highest outcome in $L$, and the lowest outcome in $H$ is also better than the lowest outcome in $L$. Even though Study 2's indirect test of double matching demonstrates that this configuration is not a necessary condition for producing violations of gain-loss separability, Payne's (2005) findings suggest that other heuristics might

operate as well. Although a comprehensive study of gain-loss separability is beyond the scope of this paper, we encourage extensions of our tests to mixed gambles with different structures. 
Thus, even though future research will surely qualify the account of mixed gambles developed here, we nevertheless see our paper as moving us a step closer toward a fuller understanding of this important and understudied choice domain. In addition, we have proposed cognitive and affective explanations for the violations of gain-loss separability but have not provided direct evidence for either explanation. The role that these psychological accounts and others play in the general evaluation of mixed gambles awaits further investigation. 


\section{References}

Abdellaoui, Mohammed (2000). "Parameter-free elicitation of utility and probability weighting functions." Management Science 46, 1497-1512.

Abdellaoui, Mohammed, Han Bleichrodt, and Corina Paraschiv (2007). "Measuring Loss Aversion: A Parameter-Free Approach." Management Science 53, 1659-1674.

Ballinger, T. Parker and Nathaniel T. Wilcox (1997). "Decisions, error and heterogeneity." Economic Journal 106, 1090-1105.

Baltussen, Guido, Thierry Post, and Pim van Vliet (2006). "Violations of Cumulative Prospect Theory in Mixed Gambles with Moderate Probabilities." Management Science 52, 1288-1290.

Barberis, Nicholas, Ming Huang, and Tano Santos (2001). "Prospect Theory and Asset Prices." Quarterly Journal of Economics 116, 1-53.

Baucells, Manel and Franz H. Heukamp (2004). "Reevaluation of the results of Levy and Levy (2002a)." Organizational Behavior and Human Decision Processes 94, 15-21.

Baucells, Manel and Frank H. Heukamp (2006). "Stochastic Dominance and Cumulative Prospect Theory." Management Science 52, 1409-23.

Bechara, Antoine, Hanna Damasio, Daniel Tranel, and Antonio R. Damasio (1997). "Deciding Advantageously Before Knowing the Advantageous Strategy." Science 275, 1293-1295.

Birnbaum, Michael H. (2004). "Causes of Allais common consequence paradoxes: An experimental dissection." Journal of Mathematical Psychology 48, 87-106.

Birnbaum, Michael H. and William R. McIntosh (1996). "Violations of Branch Independence in Choices between Gambles." Organizational Behavior and Human Decision Processes 67, 91-110.

Birnbaum, Michael H. and Jeffrey P. Bahra (2007). "Tests of Gain-loss Separability, Coalescing, Risk/Loss Attitudes, and Idempotence in Risky Decision Making." Management Science 53, 10161028.

Birnbaum, Michael H. and Juan B. Navarette (1998). "Testing descriptive utility theories: Violations of stochastic dominance and cumulative independence." Journal of Risk and Uncertainty 17, 49-78.

Bleichrodt, Han and Jose Luis Pinto (2000). "A parameter-free elicitation of the probability weighting function in medical decision analysis." Management Science 46, 1485-1496.

Breiter, Hans C., Itzhak Aharon, Daniel Kahneman, Anders Dale, and Peter Shizgal (2001). "Functional Imaging of Neural Responses to Expectancy and Experience of Monetary Gains and Losses." 
Neuron 30, 619-639.

Brenner, Lyle (2000). "Should observed overconfidence be dismissed as a statistical artifact?: Critique of Erev, Wallsten, and Budescu (1994)." Psychological Review 107, 943-946.

Brooks, Peter and Horst Zank (2005). "Loss Averse Behavior." Journal of Risk and Uncertainty 31, 301-325.

Burson, Katherine A., Richard P. Larrick, and Joshua Klayman (2006). "Skilled or unskilled, but still unaware of it: How perceptions of difficulty drive miscalibration in relative comparisons." Journal of Personality and Social Psychology, 90, 60-77.

Camerer, Colin F. (1989). "An experimental test of several generalized utility theories." Journal of Risk and Uncertainty 2, 61-104.

Camerer, Colin F. (1995). "Individual Decision Making." In The Handbook of Experimental Economics. John H. Kagel and Alvin E. Roth (Eds.). Princeton, NJ: Princeton University Press, 587-703.

Camerer, Colin (2000). "Prospect Theory in the Wild." In Choices, Values, and Frames. Daniel Kahneman and Amos Tversky (Eds.). Cambridge: Cambridge University Press, 288-300.

Camerer, Colin F. and Teck-Hua Ho (1994). "Violations of the Betweenness Axiom and Nonlinearity in Probability." Journal of Risk and Uncertainty 8, 167-196.

Camerer, Colin F. and Robin M. Hogarth (1999). "The Effects of Financial Incentives in Experiments: A Review and Capital-Labor-Production Framework." Journal of Risk and Uncertainty 19, $7-42$.

Chechile, Richard A. and Susan F. Butler (2000). "Is 'Generic Utility Theory' a Suitable Theory of Choice Behavior for Gambles with Mixed Gains and Losses?" Journal of Risk and Uncertainty 20, 189-211.

Chechile, Richard A. and Susan F. Butler (2003). "Reassessing the Testing of Generic Utility Models for Mixed Gambles." Journal of Risk and Uncertainty 26, 55-76.

Cho, Young-Hee, Lan Truong, and Mike Haneda (2005). "Testing the Indifference between a Binary Lottery and Its Edited Components Using Observed Estimates of Variability." Organizational Behavior and Human Decision Processes 97, 82-89.

Davidson, Donald, Patrick Suppes, and Sidney Siegel (1957). Decision Making: An Experimental Approach. Stanford, CA: Stanford University Press.

Erev, Ido, Thomas S. Wallsten, David V. Budescu (1994). "Simultaneous over- and underconfi- 
dence: The role of error in judgment processes." Psychological Review 101, 519-527.

Etchart-Vincent, Nathalie (2004). "Is Probability Weighting Sensitive to the Magnitude of Consequences? An Experimental Investigation on Losses." Journal of Risk and Uncertainty 38, 217-235.

Fehr-Duda, Helga, Manuele de Gennaro, and Renate Schubert (2006). "Gender, Financial Risk, and Probability Weights." Theory and Decision 60, 283-293.

Fox, Craig R. and Kelly E. See (2003). "Belief and Preference in Decision Under Uncertainty." In Thinking: Psychological Perspectives on Reasoning, Judgment and Decision Making. David Hardman and Laura Macchi (Eds.). New York: Wiley, 273-314.

Gonzalez, Richard and George Wu (1999). "On the shape of the probability weighting function." Cognitive Psychology 38, 129-166.

Hey, John D. (1995). "Experimental investigations of errors in decison-making under risk." European Economic Review 39, 633-640.

Hey, John D. and Chris Orme (1994). "Investigating generalizations of expected utility theory using experimental data." Econometrica 62, 1291-1326.

Hsee, Christopher K. and Yuval Rottenstreich (2004). "Music, Pandas, and Muggers: On the Affective Psychology of Value." Journal of Experimental Psychology: General 133, 23-30.

Hurwicz, Leonid (1951). "Optimality Criteria for Decision Making under Ignorance," Cowles Commission Discussion Paper, Statistics, No. 370.

Iverson, Geoffrey J. and Jean-Claude Falmagne (1985). "Statistical issues in measurement." Mathematical Social Sciences 10, 131-153.

Jolls, Christine, Cass Sunstein, and Richard H. Thaler (1998). "A Behavioral Approach to Law and Economics." Stanford Law Review 50, 1471-1550.

Kahneman, Daniel, Jack L. Knetsch, and Richard H. Thaler (1990). "Experimental tests of the endowment effect and the Coase Theorem." Journal of Political Economy 98, 1325-1348.

Kahneman, Daniel and Amos Tversky (1979). "Prospect theory: An analysis of decision under risk." Econometrica 47, 263-291.

Köbberling, Veronika and Peter P. Wakker (2005), "An Index of Loss Aversion." Journal of Economic Theory 122, 119-131.

Krueger, Joachim and Ross A. Mueller (2002). "Unskilled, unaware, or both? The better-thanaverage heuristic and statistical regression predict errors in estimates of own performance." Journal 
of Personality and Social Psychology 82, 180-188.

Larsen, Jeff T., A. Peter McGraw, Barbara A. Mellers, and John T. Cacioppo (2004). "The Agony of Victory and Thrill of Defeat: Mixed Emotional Reactions to Disappointing Wins and Relieving Losses." Psychological Science 15, 325-330.

Lattimore, Pamela K., Joanna K. Baker, and Ann D. Witte (1992). "The influence of probability on risky choice: A parametric examination." Journal of Economic Behavior and Organization 17, 377-400.

Levy, Haim and Moshe Levy (2002a). "Prospect Theory: Much Ado About Nothing?" Management Science 48, 1334-1349.

Levy, Haim and Moshe Levy (2002b). "Experimental test of the prospect theory value function: A stochastic dominance approach." Organizational Behavior and Human Decision Processes 89, 1058-1081.

Luce, R. Duncan (2000). Utility of Gains and Losses: Measurement-Theoretical and Experimental Approaches. Mahwah, New Jersey: Lawrence Erlbaum.

Luce, R. Duncan and Patrick Suppes (1965). "Preference, Utility, and Subjective Probability." In Handbook of Mathematical Psychology, Volume III. R. Duncan Luce, Robert B. Bush and Eugene Galanter (Eds.). New York: Wiley, 249-410.

MacCrimmon, Kenneth R. and Donald A. Wehrung (1990). "Characteristics of Risk Taking Executives." Management Science 36, 422-435.

Machina, Mark J. (1987). "Choice under Uncertainty: Problems Solved and Unsolved." Journal of Economic Perspectives 1, 121-154.

March, James and Zur Shapira (1987). "Managerial Perspectives on Risk and Risk Taking." Management Science 33, 1404-1418.

Markowitz, Harry (1952). "The utility of wealth." Journal of Political Economy 60, 151-158.

McFadden, Daniel (1981). "Econometric Models of Probabilistic Choice." In Structural Analysis of Discrete Data with Econometric Applications. Charles F. Manski and Daniel McFadden (Eds.). Cambridge, MA: MIT Press, 198-272.

McNeil, Barbara J., Steven G. Pauker, Jr. Harold C. Sox, and Amos Tversky (1982). "On the elicitation of preferences for alternative therapies." New England Journal of Medicine 306, 1259-1262.

Mood, Alexander M. and F.A. Graybill (1963). Introduction to the theory of statistics, 3rd edition. New York: McGraw-Hill.

Mosteller, Frederick and Philip Nogee (1951). "An Experimental Measurement of Utility." Journal 
of Political Economy 59, 371-404.

Myung, Jay I., George Karabatsos, and Geoffrey J. Iverson (2005). "A Bayesian approach to testing decision making axioms." Journal of Mathematical Psychology 49, 205-225.

Payne, John W. (2005). "It Is Whether You Win or Lose: The importance of the overall probabilities of winning or losing in risky choice." Journal of Risk and Uncertainty 30, 5-19.

Payne, John W., John Bettman, and Eric Johnson (1992). "Behavioral decision research: A constructive processing perspective." Annual Review of Psychology 43, 87-131.

Payne, John W. and Myron L. Braunstein (1971). "Preferences among Gambles with Equal Underlying Distributions." Journal of Experimental Psychology 87, 13-18.

Payne, John W., Daniel J. Laughhunn, and Roy L. Crum (1980). "Translation of gambles and aspiration effects in risky choice behavior." Management Science 26, 1039-1060.

Payne, John W., Daniel J. Laughhunn, and Roy L. Crum (1981). "Further tests of aspiration level effects in risky choice." Management Science 27, 953-958.

Prelec, Drazen (1998). "The probability weighting function.” Econometrica 66, 497-527.

Quattrone, George A. and Amos Tversky (1988). "Contrasting Rational and Psychological Analyses of Political Choice." American Political Science Review 82, 719-736.

Quiggin, John (1982). "A Theory of Anticipated Utility." Journal of Economic Behavior and Organization 3, 323-343.

Rottenstreich, Yuval and Christopher K. Hsee (2001). "Money, kisses, and electric shocks: On the affective psychology of risk." Psychological Science 12, 185-190.

Rottenstreich, Yuval and Suzanne Shu (2004). "The Connections between Affect and Decision Making: Nine Resulting Phenomena." In Blackwell Handbook of Judgment and Decision Making. Derek J. Koehler and Nigel Harvey (Eds.). Oxford: Blackwell, 444-463.

Schmidt, Ulrich and Stefan Traub (2002). "An Experimental Test of Loss Aversion." Journal of Risk and Uncertainty 25, 233-249.

Slovic, Paul (1967). "The relative influence of probabilities and payoffs upon the perceived risk of a gamble." Psychonomic Science 9, 223-224.

Slovic, Paul, Melissa Finucane, Ellen Peters, and Donald G. MacGregor (2002). "The Affect Heuristic." In Heuristics and Biases: The Psychology of Human Judgment. Thomas Gilovich, Dale Griffin and Daniel Kahneman (Eds.). Cambridge, England: Cambridge University Press, 397-420.

Slovic, Paul and Lichtenstein, Sarah (1968). "Relative Importance of Probabilities and Payoffs in 
Risk Taking." Journal of Experimental Psychology 78, 1-18.

Starmer, Chris (2000). "Developments in Non-Expected Utility Theory: The Hunt for a Descriptive Theory of Choice under Risk." Journal of Economic Literature 38, 332-382.

Thaler, Richard H. and Eric J. Johnson (1990). "Gambling with the house money and trying to break even: The effects of prior outcomes on risky choice." Management Science 36, 643-660.

Tversky, Amos (1969). "Intransitivity of preferences." Psychological Review 76, 31-48.

Tversky, Amos (1972). "Elimination by aspects: A theory of choice." Psychological Review 79, 281-299.

Tversky, Amos and Craig R. Fox (1995). "Weighing Risk and Uncertainty." Psychological Review 102, 269-283.

Tversky, Amos and Daniel Kahneman (1992). "Advances in Prospect Theory: Cumulative Representation of Uncertainty." Journal of Risk and Uncertainty 5, 297-323.

Wakker, Peter P. (2003). "The Data of Levy and Levy (2002), 'Prospect Theory: Much Ado about Nothing?' Support Prospect Theory." Management Science 49, 979-981.

Wakker, Peter P. and Amos Tversky (1993). "An Axiomatization of Cumulative Prospect Theory." Journal of Risk and Uncertainty 7, 147-176.

Wu, George (1994). "An Empirical Test of Ordinal Independence." Journal of Risk and Uncertainty 9, 39-60.

Wu, George and Richard Gonzalez (1996). "Curvature of the Probability Weighting Function." Management Science 42, 1676-90.

Wu, George, Jiao Zhang and Mohammed Abdellaoui (2005). "Testing prospect theories using probability tradeoff consistency." Journal of Risk and Uncertainty 30, 107-131.

Wu, George, Jiao Zhang, and Richard Gonzalez (2004). "Decision under Risk." In Blackwell Handbook of Judgment and Decision Making. Derek J. Koehler and Nigel Harvey (Eds.). Oxford: Blackwell, 399-423. 
This page is intentionally blank. Proper e-companion title page, with INFORMS branding and exact metadata of the main paper, will be produced by the INFORMS office when the issue is being assembled. 


\section{Introduction}

This paper supplements Wu and Markle (2007). In Section EC.1, we develop in greater detail the error model presented in Section 3.3 of that paper. In Section EC.2, we derive implications of these error models on various measures of double matching violations. In Section EC.3, we examine these four measures empirically. Section EC.4 details the likelihood-ratio test used to analyze the Study 1 data. Section EC.5 presents robustness analyses of our fits of the probability weighting function.

We then present some supplementary material for Study 2 of Wu and Markle (2007). Section EC.6 details the stochastic choice procedure used to test gain-loss separability for Study 2, and Section EC.7 presents robustness analyses of the probability weighting function estimation for Study 2. Section EC.8 concludes.

For completeness and to improve readability, this companion paper repeats some of the text from $\mathrm{Wu}$ and Markle.

\section{EC.1. Error Models}

The goal of this error analysis is to examine whether the empirical pattern of double matching violations in Study 1 could be explained by random error. Recall Problems 1 through 3 (also Study 1, Test 7) from the Introduction of Wu and Markle:

Problem $1(n=81)$ :

$$
H=\left(\begin{array}{c}
.50 \text { chance at } \$ 4200 \\
.50 \text { chance at } \$-3000
\end{array}\right) \text { vs. } L=\left(\begin{array}{c}
.75 \text { chance at } \$ 3000 \\
.25 \text { chance at } \$-4500
\end{array}\right)
$$

Problem $2(n=81)$ : $[52 \%]$

$[48 \%]$

$$
H^{+}=\left(\begin{array}{c}
.50 \text { chance at } \$ 4200 \\
.50 \text { chance at } \$ 0 \\
{[15 \%]}
\end{array}\right) \text { vs. } L^{+}=\left(\begin{array}{c}
.75 \text { chance at } \$ 3000 \\
.25 \text { chance at } \$ 0 \\
{[85 \%]}
\end{array}\right)
$$

Problem $3(n=81)$ :

$$
H^{-}=\left(\begin{array}{c}
.50 \text { chance at } \$ 0 \\
.50 \text { chance at } \$-3000
\end{array}\right) \text { vs. } L^{-}=\left(\begin{array}{c}
.75 \text { chance at } \$ 0 \\
.25 \text { chance at } \$-4500
\end{array}\right)
$$

We suggested that the modal pattern of choices, $H, L^{+}$, and $L^{-}$, was consistent with a process in which decision makers were less sensitive to probability differences for mixed gambles than for either gain or loss gambles. In Study 1, our primary empirical investigation of double matching, we found that $\% H>\frac{\% H^{+}+\% H^{-}}{2}$ for 29 of our 34 Study 1 tests, all of which had the same basic structure as Problems 1 through 3.

Could this pattern of data, however, be an artifact of random error? To investigate this possibility, we compare a random error model with several models in which the error is "systematic." We show that the random error model cannot account for the full set of data presented in Wu and Markle. On the other hand, a model that employs systematic or asymmetric error and is consistent with the intuition suggested by our introductory example captures the basic qualitative patterns found in our data.

We outline the basic assumptions underlying the proposed error models. We consider assumptions about preference types, satisfaction of the double matching axiom, and error rates.

\section{EC.1.1. Preference Types}

We assume that there are four types, $\theta_{H^{+} H^{-}}, \theta_{H^{+} L^{-}}, \theta_{L^{+} H^{-}}$, and $\theta_{L^{+} L^{-}}$. Each type has "underlying preferences," though their "revealed preferences" reflect some error (see Section EC.1.3 below). Type $\theta_{H^{+} H^{-}}$prefers $H^{+}$over $L^{+}$and $H^{-}$over $L^{-}$, type $\theta_{H^{+} L^{-}}$prefers $H^{+}$over $L^{+}$and $L^{-}$over $H^{-}$, type $\theta_{L^{+} H^{-}}$prefers $L^{+}$over $H^{+}$and $H^{-}$over $L^{-}$, and type $\theta_{L^{+} L^{-}}$prefers $L^{+}$over $H^{+}$and $L^{-}$over $H^{-}$. The probabilities of the four types are given by $p_{H^{+} H^{-}}, p_{H^{+} L^{-}}, p_{L^{+} H^{-}}$, and $p_{L^{+} L^{-}}$, or $p_{H H}, p_{H L}, p_{L H}$, and $p_{L L}$ for short. Table EC.1 summarizes the assumptions about types. 


$\begin{gathered}\text { Table EC.1 Probabilities of } \\
\text { combinations of preferences for } \\
\text { the types described in } \\
\text { Section EC.1.1. }\end{gathered}$
\begin{tabular}{|l|c|c|}
\hline & $H^{-} \succ L^{-}$ & $L^{-} \succ H^{-}$ \\
\hline$H^{+} \succ L^{+}$ & $p_{H^{+} H^{-}}$ & $p_{H^{+} L^{-}}$ \\
\hline$L^{+} \succ H^{+}$ & $p_{L^{+} H^{-}}$ & $p_{L^{+} L^{-}}$ \\
\hline
\end{tabular}

\section{EC.1.2. Double Matching}

We assume that "underlying preferences" satisfy double matching. Type $\theta_{H^{+} H^{-}}$prefers $H^{+}$over $L^{+}$and $H^{-}$over $L^{-}$, and thus $H$ over $L$, whereas type $\theta_{L^{+} L^{-}}$prefers $L^{+}$over $H^{+}$and $L^{-}$over $H^{-}$, and hence $L$ over $H$. We consider the other two types, $\theta_{H^{+} L^{-}}$and $\theta_{L^{+} H^{-}}$, "indeterminate" and assume that they choose according to their gain or loss preferences with equal probability (see below).

\section{EC.1.3. Error Rates}

We also assume that decision makers choose with error. Let $\epsilon_{S}$ be the error rate for single-domain gambles, such that $\epsilon_{S}=P\left(L^{+} \succ H^{+} \mid \theta_{H^{+} H^{-}}\right)=P\left(L^{+} \succ H^{+} \mid \theta_{H^{+} L^{-}}\right)=P\left(L^{-} \succ H^{-} \mid \theta_{H^{+} H^{-}}\right)=$ $P\left(H^{+} \succ L^{+} \mid \theta_{L^{+} L^{-}}\right)=P\left(H^{-} \succ L^{-} \mid \theta_{L^{+} L^{-}}\right)$, etc. The remaining conditional probabilities are defined analogously.

We consider several error models for the choice between mixed gambles $H$ and $L$. Let $\epsilon_{L}=P(L \succ$ $\left.H \mid \theta_{H^{+} H^{-}}\right)$and $\epsilon_{H}=P\left(H \succ L \mid \theta_{L^{+} L^{-}}\right)$. We assume that the two indeterminate types, $\theta_{H^{+} L^{-}}$and $\theta_{L^{+} H^{-}}$, choose like type $\theta_{H^{+} H^{-}}$or type $\theta_{L^{+} L^{-}}$with equal probability. Therefore, $P\left(H \succ L \mid \theta_{H^{+} L^{-}}\right)=$ $P\left(H \succ L \mid \theta_{L^{+} H^{-}}\right)=(1 / 2) P\left(H \succ L \mid \theta_{H^{+} H^{-}}\right)+(1 / 2) P\left(H \succ L \mid \theta_{L^{+} L^{-}}\right)=(1 / 2)\left(1-\epsilon_{L}\right)+(1 / 2)\left(\epsilon_{H}\right)$.

The four models below make different restrictions on the relationship among the four error rates, $\epsilon_{S}, \epsilon_{L}, \epsilon_{H}$ :

"Null" model: $\epsilon_{S}=\epsilon_{L}=\epsilon_{H}$.

"Mixed" error model: $\epsilon_{S} \leq \epsilon_{L}=\epsilon_{H}$.

"Asymmetric" error model: $\epsilon_{S}=\epsilon_{L} \leq \epsilon_{H}$.

"General" error model: $\epsilon_{S}, \epsilon_{L}, \epsilon_{H}$.

The null error model assumes that the same error rate applies to all gambles, whereas the mixed error model permits a different error rate for mixed gambles than single-domain gambles. The asymmetric model allows a different error rate for type $\theta_{L^{+} L^{-}}$than type $\theta_{H^{+} H^{-}}$. The empirical pattern observed in Study 1 of $\mathrm{Wu}$ and Markle $(2007), \% H>\max \left(\% H^{+}, \% H^{-}\right)$, as well as the hypothesized process, suggests that $\epsilon_{H}>\epsilon_{L}=\epsilon_{S}$ for the asymmetric model. The general model allows all three error rates to differ.

Note also that the indeterminate types, $\theta_{H^{+} L^{-}}$and $\theta_{L^{+} H^{-}}$, reflect the error structures posited in each of these models. Thus, $P\left(H \succ L \mid \theta_{H^{+} L^{-}}\right)=\frac{1}{2} P\left(H \succ L \mid \theta_{H^{+} H^{-}}\right)+\frac{1}{2} P\left(H \succ L \mid \theta_{L^{+} L^{-}}\right)=\frac{1}{2}(1-$ $\left.\epsilon_{S}\right)+\frac{1}{2}\left(\epsilon_{H}\right)=\frac{1}{2}\left(1-\epsilon_{S}+\epsilon_{H}\right)$.

\section{EC.2. Implications of error models}

We develop four implications of the null, mixed, and asymmetric error models. We assume the type structure depicted in Table EC.1. We also assume that all error rates are between 0 and $\frac{1}{2}$ : $0<\epsilon_{S}<\frac{1}{2}, 0<\epsilon_{H}<\frac{1}{2}$, and $0 \leq \epsilon_{L}<\frac{1}{2}$.

We consider implications of each error model on: (i) choice percentages for single-domain gambles and mixed gambles; (ii) double matching violation rates; (iii) the relationship between error rates, $P\left(H \mid L^{+} L^{-}\right)$and $P\left(L \mid H^{+} H^{-}\right)$; and (iv) the likelihood of choosing $H$ for the "indeterminate" patterns, $H^{+} L^{-}$and $L^{+} H^{-}$. The derivations below are summarized in Table EC.2. Note that the 
Table EC.2 Qualitative implications of the null, mixed, and asymmetric error models. These implications are depicted graphically in Figures EC.1 through EC.3.

\begin{tabular}{|c|c|c|c|}
\hline & Null error model & Mixed error model & Asymmetric error model \\
\hline $\begin{array}{ll}\text { Restriction } & \text { on } \\
\text { error rates } & \end{array}$ & $\epsilon_{S}=\epsilon_{L}=\epsilon_{H}$ & $\epsilon_{H}=\epsilon_{L}>\epsilon_{S}$ & $\epsilon_{H}>\epsilon_{S}=\epsilon_{L}$ \\
\hline $\begin{array}{l}\text { Choice percent- } \\
\text { ages }\end{array}$ & $\begin{array}{l}P(H) \text { vs. } \frac{P\left(H^{+}\right)+P\left(H^{-}\right)}{2} \\
\bullet=\text { if } p_{H^{+} H^{-}}<p_{L^{+} L^{-}} \\
\bullet=\text { if } p_{H^{+} H^{-}}=p_{L^{+} L^{-}} \\
\bullet=\text {, if } p_{H^{+} H^{-}}>p_{L^{+} L^{-}}\end{array}$ & $\begin{array}{l}P(H) \text { vs. } \frac{P\left(H^{+}\right)+P\left(H^{-}\right)}{2} \\
\bullet>\text {, if } p_{H^{+} H^{-}}<p_{L^{+} L^{-}} \\
\bullet=\text {, if } p_{H^{+} H^{-}}=p_{L^{+} L^{-}} \\
\bullet<\text {, if } p_{H^{+} H^{-}}>p_{L^{+} L^{-}}\end{array}$ & $\begin{array}{l}P(H) \text { vs. } \frac{P\left(H^{+}\right)+P\left(H^{-}\right)}{2} \\
\bullet>\text {, if } p_{H^{+} H^{-}}<p_{L^{+} L^{-}} \\
\bullet>\text {, if } p_{H^{+} H^{-}}=p_{L^{+} L^{-}} \\
\bullet>\text {, if } p_{H^{+} H^{-}}>p_{L^{+} L^{-}}\end{array}$ \\
\hline $\begin{array}{l}\text { Double matching } \\
\text { violation rates }\end{array}$ & $\begin{array}{c}P\left(H L^{+} L^{-}\right) \text {vs. } P\left(L^{+} H^{-}\right) \\
\bullet>, \text { if } p_{H^{+} H^{-}}<p_{L^{+} L^{-}} \\
\bullet=, \text { if } p_{H^{+} H^{-}}=p_{L^{+} L^{-}} \\
\bullet<, \text { if } p_{H^{+} H^{-}}>p_{L^{+} L^{-}}\end{array}$ & $\begin{array}{c}P\left(H L^{+} L^{-}\right) \text {vs. } P\left(L H^{+} H^{-}\right) \\
\bullet>\text {, if } p_{H^{+} H^{-}}<p_{L^{+} L^{-}} \\
\bullet=\text {, if } p_{H^{+} H^{-}}=p_{L^{+} L^{-}} \\
\bullet<, \text { if } p_{H^{+} H^{-}}>p_{L^{+} L^{-}}\end{array}$ & $\begin{array}{l}P\left(H L^{+} L^{-}\right) \text {vs. } P\left(L H^{+} H^{-}\right) \\
\bullet>\text {, if } p_{H^{+} H^{-}}<p_{L^{+} L^{-}} \\
\bullet>\text {, if } p_{H^{+} H^{-}}=p_{L^{+} L^{-}} \\
\bullet>\text { then }<\text {, if } p_{H^{+} H^{-}}> \\
p_{L^{+} L^{-}}\end{array}$ \\
\hline $\begin{array}{l}\text { Error rates } \\
P\left(H \mid L^{+} L^{-}\right) \text {and } \\
P\left(L \mid H^{+} H^{-}\right)\end{array}$ & $\begin{array}{l}P\left(H \mid L^{+} L^{-}\right) \\
P\left(L \mid H^{+} H^{-}\right) \\
\bullet<, \text { if } p_{H^{+} H^{-}}<p_{L^{+} L^{-}} \\
\bullet=\text { if } p_{H^{+} H^{-}}=p_{L^{+} L^{-}} \\
\bullet>\text {, if } p_{H^{+} H^{-}}>p_{L^{+} L^{-}}\end{array}$ & $\begin{array}{l}P\left(H \mid L^{+} L^{-}\right) \\
P\left(L \mid H^{+} H^{-}\right) \\
\bullet<, \text { if } p_{H^{+} H^{-}}<p_{L^{+} L^{-}} \\
\bullet=, \text { if } p_{H^{+} H^{-}}=p_{L^{+} L^{-}} \\
\bullet>\text {, if } p_{H^{+} H^{-}}>p_{L^{+} L^{-}}\end{array}$ & $\begin{array}{l}P\left(H \mid L^{+} L^{-}\right) \\
P\left(L \mid H^{+} H^{-}\right) \\
\quad<<\text { then }>\text {, if } p_{H^{+} H^{-}}< \\
p_{L^{+} L^{-}} \\
\quad \bullet>\text {, if } p_{H^{+} H^{-}}=p_{L^{+} L^{-}} \\
\quad \bullet>\text { if } p_{H^{+} H^{-}}>p_{L^{+} L^{-}}\end{array}$ \\
\hline $\begin{array}{l}\text { Likelihood of } \\
\text { choosing } H \text { for } \\
\text { "indeterminate" } \\
\text { patterns }\end{array}$ & $\begin{array}{l}P\left(H \mid H^{+} L^{-}\right) \\
P\left(H \mid L^{+} H^{-}\right) \text {vs. } .5 \\
\bullet<, \text { if } p_{H^{+} H^{-}}<p_{L^{+} L^{-}} \\
\bullet=, \text { if } p_{H^{+} H^{-}}=p_{L^{+} L^{-}} \\
\bullet>\text {, if } p_{H^{+} H^{-}}>p_{L^{+} L^{-}}\end{array}$ & $\begin{array}{l}P\left(H \mid H^{+} L^{-}\right) \\
P\left(H \mid L^{+} H^{-}\right) \text {vs. } .5 \\
\bullet<, \text { if } p_{H^{+} H^{-}}<p_{L^{+} L^{-}} \\
\bullet=\text {, if } p_{H^{+} H^{-}}=p_{L^{+} L^{-}} \\
\bullet>\text {, if } p_{H^{+} H^{-}}>p_{L^{+} L^{-}}\end{array}$ & $\begin{array}{l}P\left(H \mid H^{+} L^{-}\right) \\
P\left(H \mid L^{+} H^{-}\right) \text {vs. } .5 \\
\bullet<\text { then }>\text {, if } p_{H^{+} H^{-}}< \\
p_{L^{+} L^{-}} \\
\quad>\text {, if } p_{H^{+} H^{-}}=p_{L^{+} L^{-}} \\
\bullet>\text {, if } p_{H^{+} H^{-}}>p_{L^{+} L^{-}}\end{array}$ \\
\hline
\end{tabular}

null and the mixed error models have identical qualitative implications for all measures except for choice percentages. Most critically, both models require a symmetry around $p_{H H}=p_{L L}$, whereas the asymmetric model does not.

\section{EC.2.1. Implications of null error model}

We derive the implications of the null error model on the four quantities described above. We denote $0<\epsilon=\epsilon_{S}=\epsilon_{L}=\epsilon_{H}<.5$, the probability of an error, i.e., $\epsilon=P\left(H^{+} \succ L^{+} \mid \theta_{L^{+} L^{-}}\right)=P\left(H^{-} \succ\right.$ $\left.L^{-} \mid \theta_{L^{+} L^{-}}\right)=P\left(H \succ L \mid \theta_{L^{+} L^{-}}\right)$, and $1-\epsilon=P\left(L^{+} \succ H^{+} \mid \theta_{L^{+} L^{-}}\right)=P\left(L^{-} \succ H^{-} \mid \theta_{L^{+} L^{-}}\right)=P(L \succ$ $\left.H \mid \theta_{L^{+} L^{-}}\right)$, etc.

We depict the results of our derivations graphically in Figure EC.1. To simplify the graphical presentation and clarify the implications, we assume that $p_{H L}=p_{L H}=0$.

EC.2.1.1. Choice percentages First, we consider the implication of the null error model for choice percentages. Let $P(H), P\left(H^{+}\right)$, and $P\left(H^{-}\right)$be the expected choice percentages for mixed gamble $H$, gain gamble $H^{+}$, and loss gamble $H^{-}$, respectively. Then, $P\left(H^{+}\right)=\left(p_{H H}+p_{H L}\right)(1-$ $\epsilon)+\left(p_{L H}+p_{L L}\right)(\epsilon), P\left(H^{-}\right)=\left(p_{H H}+p_{L H}\right)(1-\epsilon)+\left(p_{H L}+p_{L L}\right)(\epsilon)$, and $P(H)=\left(p_{H H}\right)(1-\epsilon)+$ $\frac{1}{2}\left(p_{H L}+p_{L H}\right)+\left(p_{L L}\right)(\epsilon)$. It is easy to see that $P(H)=\frac{P\left(H^{+}\right)+P\left(H^{-}\right)}{2}$.

Figure EC.1A plots the choice percentage, $P(H)$ as a function of the average of $P\left(H^{+}\right)$and $P\left(H^{-}\right)$. Note that the minimum and maximum choice percentages are limited by the error rate, $\epsilon$. For example, if $p_{H H}=1$, then $P\left(H^{+}\right)=P\left(H^{-}\right)=P(H)=1-\epsilon$. 
Figure EC.1 Implications of null error model on choice percentages, double matching violation rates, error rates, and conditional probabilities.
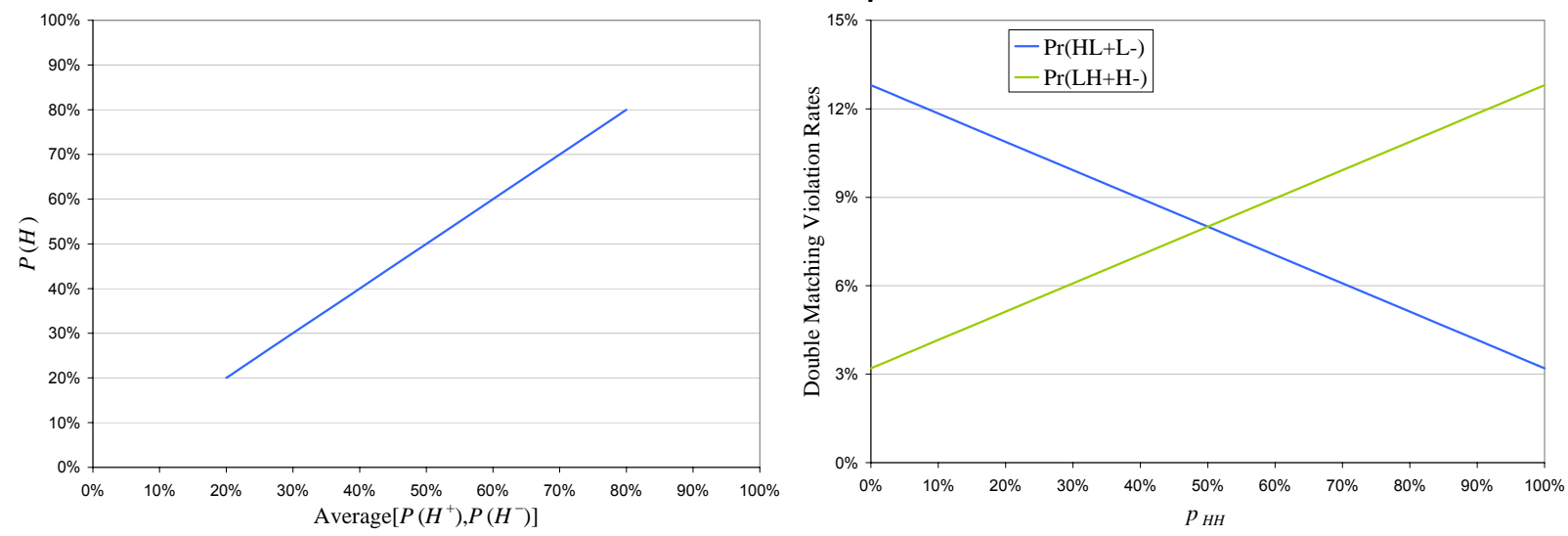

A: Choice Percentages

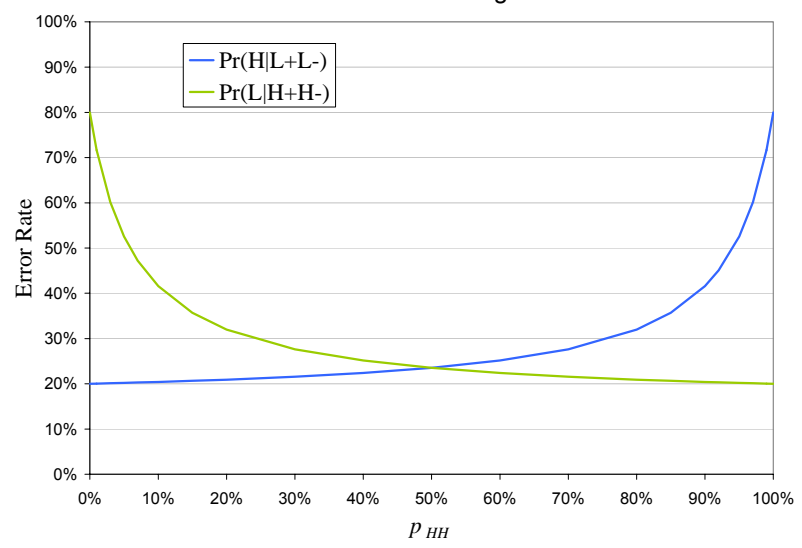

C: Error Rates

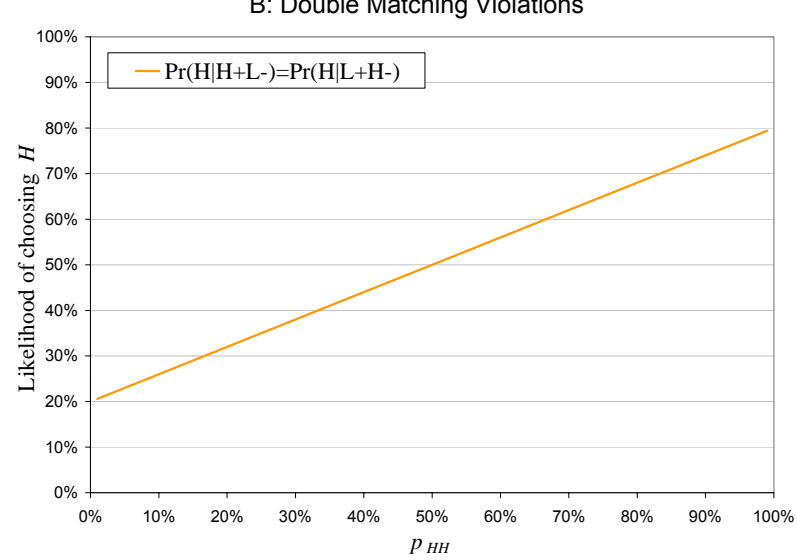

D: Conditional Probabilities

Note. To simplify the presentation, we assume that $p_{H L}=p_{L H}=0$ and $\epsilon_{S}=\epsilon_{H}=\epsilon_{L}=.2$. Panel A illustrates expected choice percentages, $P(H)$, as a function of the average of $P\left(H^{+}\right)$and $P\left(H^{-}\right)$. The null error model requires that $P(H)$ be identical to $\frac{P\left(H^{+}\right)+P\left(H^{-}\right)}{2}$. Panel B depicts double matching violation rates, $P\left(H L^{+} L^{-}\right)$and $P\left(L H^{+} H^{-}\right)$, as a function of $p_{H H}$, the probability of type $\theta_{H^{+} H^{-}}$. The null error model requires that double matching violations $H L^{+} L^{-}$exceed double matching violations $L H^{+} H^{-}$if $p_{H H}<p_{L L}$, but that the reverse holds for $p_{H H}>p_{L L}$. Panel C shows error rates, $P\left(H \mid L^{+} L^{-}\right)$and $P\left(L \mid H^{+} H^{-}\right)$, as a function of the frequency of $p_{H H}$. The null error model requires that error rate $P\left(L \mid H^{+} H^{-}\right)$exceed $P\left(H \mid L^{+} L^{-}\right)$for $p_{H H}<p_{L L}$, with the reverse holding for $p_{H H}>p_{L L}$. Panel D illustrates conditional probabilities, $P\left(H \mid H^{+} L^{-}\right)$and $P\left(H \mid L^{+} H^{-}\right)$as a function of $p_{H H}$. The null error model requires that the likelihood of choosing $H$ given one of the indeterminate patterns, $H^{+} L^{-}$and $L^{+} H^{-}$, be less than $\frac{1}{2}$ if $p_{H H}<p_{L L}$ and greater than $\frac{1}{2}$ if $p_{H H}>p_{L L}$.

EC.2.1.2. Double Matching Violation Rates We show how the frequency of the two types of violations depends on the frequency of the various types. Recall that the two types of double matching violations $H L^{+} L^{-}$and $L H^{+} H^{-}$with frequencies denoted $P\left(H L^{+} L^{-}\right)$and $P\left(L H^{+} H^{-}\right)$. Since,

$$
\begin{aligned}
P\left(H L^{+} L^{-}\right)= & P\left(H L^{+} L^{-} \mid \theta_{H^{+} H^{-}}\right) P\left(\theta_{H^{+} H^{-}}\right)+P\left(H L^{+} L^{-} \mid \theta_{H^{+} L^{-}}\right) P\left(\theta_{H^{+} L^{-}}\right)+ \\
& P\left(H L^{+} L^{-} \mid \theta_{L^{+} H^{-}}\right) P\left(\theta_{L^{+} H^{-}}\right)+P\left(H L^{+} L^{-} \mid \theta_{L^{+} L^{-}}\right) P\left(\theta_{L^{+} L^{-}}\right) \\
= & p_{H H}(1-\epsilon)\left(\epsilon^{2}\right)+\frac{1}{2} p_{H L}(\epsilon)(1-\epsilon)+\frac{1}{2} p_{L H}(\epsilon)(1-\epsilon)+p_{L L}(1-\epsilon)^{2}(\epsilon),
\end{aligned}
$$

and

$$
P\left(L H^{+} H^{-}\right)=p_{H H}(1-\epsilon)^{2}(\epsilon)+\frac{1}{2} p_{H L}(\epsilon)(1-\epsilon)+\frac{1}{2} p_{L H}(\epsilon)(1-\epsilon)+p_{L L}(1-\epsilon)\left(\epsilon^{2}\right),
$$


$P\left(H L^{+} L^{-}\right)>P\left(L H^{+} H^{-}\right)$if $\left(p_{L L}-p_{H H}\right)(1-\epsilon)(\epsilon)(1-2 \epsilon)>0$. Thus, if $\epsilon<.5, P\left(H L^{+} L^{-}\right)>$ $P\left(L H^{+} H^{-}\right)$if $p_{H H}<p_{L L}$; otherwise, $P\left(L H^{+} H^{-}\right)>P\left(H L^{+} L^{-}\right)$if $p_{H H}>p_{L L}$.

Figure EC.1B plots the rates of double matching violations of the two types as a function of $p_{H H}$.

EC.2.1.3. Error rates: $P\left(H \mid L^{+} L^{-}\right)$and $P\left(L \mid H^{+} H^{-}\right)$We next consider the implication for the null error model for the comparison of the error rates, $P\left(H \mid L^{+} L^{-}\right)$and $P\left(L \mid H^{+} H^{-}\right)$. To show that these error rates depend on the probability of the different types, note that

$$
\begin{aligned}
P\left(H \mid L^{+} L^{-}\right)= & P\left(H \mid \theta_{H^{+} H^{-}}\right) P\left(\theta_{H^{+} H^{-}} \mid L^{+} L^{-}\right)+P\left(H \mid \theta_{H^{+} L^{-}}\right) P\left(\theta_{H^{+} L^{-}} \mid L^{+} L^{-}\right)+ \\
& P\left(H \mid \theta_{L^{+} H^{-}}\right) P\left(\theta_{L^{+} H^{-}} \mid L^{+} L^{-}\right)+P\left(H \mid \theta_{L^{+} L^{-}}\right) P\left(\theta_{H^{+} H^{-}} \mid L^{+} L^{-}\right) .
\end{aligned}
$$

Since $P\left(L^{+} L^{-}\right)=p_{H H}\left(\epsilon^{2}\right)+p_{H L}(\epsilon)(1-\epsilon)+p_{L H}(1-\epsilon)(\epsilon)+p_{L L}(1-\epsilon)^{2}, P\left(\theta_{H^{+} H^{-}} \mid L^{+} L^{-}\right)=\frac{p_{H H}\left(\epsilon^{2}\right)}{P\left(L^{+} L^{-}\right)}$, $P\left(\theta_{H^{+} L^{-}} \mid L^{+} L^{-}\right)=\frac{p_{H L}(\epsilon)(1-\epsilon)}{P\left(L^{+} L^{-}\right)}, P\left(\theta_{L^{+} H^{-}} \mid L^{+} L^{-}\right)=\frac{p_{L H}(1-\epsilon)(\epsilon)}{P\left(L^{+} L^{-}\right)}$, and $P\left(\theta_{L^{+} L^{-}} \mid L^{+} L^{-}\right)=\frac{p_{L L}(1-\epsilon)^{2}}{P\left(L^{+} L^{-}\right)}$, therefore,

$$
P\left(H \mid L^{+} L^{-}\right)=\frac{p_{H H}(1-\epsilon)\left(\epsilon^{2}\right)+\frac{1}{2} p_{H L}(\epsilon)(1-\epsilon)+\frac{1}{2} p_{L H}(1-\epsilon)(\epsilon)+p_{L L}(\epsilon)(1-\epsilon)^{2}}{p_{H H}\left(\epsilon^{2}\right)+p_{H L}(\epsilon)(1-\epsilon)+p_{L H}(1-\epsilon)(\epsilon)+p_{L L}(1-\epsilon)^{2}} .
$$

Similarly,

$$
P\left(L \mid H^{+} H^{-}\right)=\frac{p_{H H}(\epsilon)(1-\epsilon)^{2}+\frac{1}{2} p_{H L}(\epsilon)(1-\epsilon)+\frac{1}{2} p_{L H}(1-\epsilon)(\epsilon)+p_{L L}(1-\epsilon)\left(\epsilon^{2}\right)}{p_{H H}(1-\epsilon)^{2}+p_{H L}(\epsilon)(1-\epsilon)+p_{L H}(1-\epsilon)(\epsilon)+p_{L L}\left(\epsilon^{2}\right)} .
$$

It is easy to see that $P\left(H \mid L^{+} L^{-}\right)=P\left(L \mid H^{+} H^{-}\right)$if $p_{H H}=p_{L L}$. To show that $P\left(H \mid L^{+} L^{-}\right)>$ $P\left(L \mid H^{+} H^{-}\right)$if $p_{H H}>p_{L L}$ and that $P\left(L \mid H^{+} H^{-}\right)>P\left(H \mid L^{+} L^{-}\right)$if $p_{H H}<p_{L L}$, we simplify $P\left(H \mid L^{+} L^{-}\right)>P\left(L \mid H^{+} H^{-}\right)$to get:

$$
\left(p_{H H}-p_{L L}\right)(1-2 \epsilon)\left[\left(p_{H H}+p_{L L}\right)(1-\epsilon)(\epsilon)+\left(p_{H L}+p_{L H}\right)\left(\frac{1}{2}-(1-\epsilon)(\epsilon)\right)\right]>0,
$$

which holds if $p_{H H}>p_{L L}$ and $\epsilon<.5$. Reversing the sign of Eq. (1) gives $P\left(L \mid H^{+} H^{-}\right)>P\left(H \mid L^{+} L^{-}\right)$ if $p_{H H}<p_{L L}$.

Figure EC.1C plots $P\left(H \mid L^{+} L^{-}\right)$and $P\left(L \mid H^{+} H^{-}\right)$as a function of $p_{H H}$, assuming $\epsilon=.2$.

EC.2.1.4. Likelihood of choosing $H$ for "indeterminate" patterns Finally, we consider the likelihood of choosing $H$ given the "indeterminate" patterns, $H^{+} L^{-}$and $L^{+} H^{-}$. Note that

$$
\begin{aligned}
P\left(H \mid H^{+} L^{-}\right)= & P\left(H \mid \theta_{H^{+} H^{-}}\right) P\left(\theta_{H^{+} H^{-}} \mid H^{+} L^{-}\right)+P\left(H \mid \theta_{H^{+} L^{-}}\right) P\left(\theta_{H^{+} L^{-}} \mid H^{+} L^{-}\right)+ \\
& P\left(H \mid \theta_{L^{+} H^{-}}\right) P\left(\theta_{L^{+} H^{-}} \mid H^{+} L^{-}\right)+P\left(H \mid \theta_{L^{+} L^{-}}\right) P\left(\theta_{H^{+} H^{-}} \mid H^{+} L^{-}\right) .
\end{aligned}
$$

Then, $P\left(H^{+} L^{-}\right)=p_{H H}(1-\epsilon)(\epsilon)+p_{H L}(1-\epsilon)^{2}+p_{L H}\left(\epsilon^{2}\right)+p_{L L}(1-\epsilon)(\epsilon)$, and, therefore,

$$
P\left(H \mid H^{+} L^{-}\right)=\frac{p_{H H}(1-\epsilon)^{2}(\epsilon)+\frac{1}{2} p_{H L}(1-\epsilon)^{2}+\frac{1}{2} p_{L H}\left(\epsilon^{2}\right)+p_{L L}\left(\epsilon^{2}\right)(1-\epsilon)}{p_{H H}(1-\epsilon)(\epsilon)+p_{H L}(1-\epsilon)^{2}+p_{L H}\left(\epsilon^{2}\right)+p_{L L}(1-\epsilon)(\epsilon)} .
$$

Thus, $P\left(H \mid H^{+} L^{-}\right)>\frac{1}{2}$ if $\left(p_{H H}-p_{L L}\right)(1-\epsilon)(\epsilon)(1-2 \epsilon)>0$, which holds if $\epsilon<.5$ and $p_{H H}>$ $p_{L L}$. Reversing the sign, we get $P\left(H \mid H^{+} L^{-}\right)<\frac{1}{2}$ if $p_{H H}<p_{L L}$, provided that $\epsilon<.5$. A similar manipulation shows that $P\left(H \mid L^{+} H^{-}\right)>\frac{1}{2}$ if $\epsilon<.5$ and $p_{H H}>p_{L L}$, whereas $P\left(H \mid L^{+} H^{-}\right)<\frac{1}{2}$ if $\epsilon<.5$ and $p_{H H}<p_{L L}$.

Figure EC.1D plots $P\left(H \mid H^{+} L^{-}\right)$and $P\left(H \mid L^{+} H^{-}\right)$as a function of $p_{H H}$, assuming $\epsilon=.2$. 


\section{EC.2.2. Implications of mixed error model}

We next consider the implications of the mixed error model for the measures analyzed in the previous subsection. Again, we examine how these measures vary as a function of the probability of the four types in Table EC.1. Recall that the mixed error model allows a different error rate to govern single-domain gambles and mixed gambles. We denote $\epsilon_{M}=\epsilon_{H}=\epsilon_{L}<.5$ the error rate for mixed gambles. Our introductory example is consistent with $\epsilon_{S}<\epsilon_{M}$.

EC.2.2.1. Choice percentages We begin by considering the implication of the mixed error model for choice percentages, $P(H), P\left(H^{+}\right)$, and $P\left(H^{-}\right)$. Since, $P\left(H^{+}\right)=\left(p_{H H}+p_{H L}\right)\left(1-\epsilon_{S}\right)+$ $\left(p_{L H}+p_{L L}\right)\left(\epsilon_{S}\right), P\left(H^{-}\right)=\left(p_{H H}+p_{L H}\right)\left(1-\epsilon_{S}\right)+\left(p_{H L}+p_{L L}\right)\left(\epsilon_{S}\right)$, and $P(H)=\left(p_{H H}\right)\left(1-\epsilon_{M}\right)+$ $\frac{1}{2}\left(p_{H L}+p_{L H}\right)+\left(p_{L L}\right)\left(\epsilon_{M}\right), P(H)>\frac{P\left(H^{+}\right)+P\left(H^{-}\right)}{2}=\left(p_{H H}\right)\left(1-\epsilon_{S}\right)+\frac{1}{2}\left(p_{H L}+p_{L H}\right)+\left(p_{L L}\right)\left(\epsilon_{S}\right)$ if $\epsilon_{M}>\epsilon_{S}$ and $p_{H H}<p_{L L}$, whereas $P(H)<\frac{P\left(H^{+}\right)+P\left(H^{-}\right)}{2}$ if $\epsilon_{M}>\epsilon_{S}$ and $p_{H H}>p_{L L}$. Figure EC.2A plots the choice percentage, $P(H)$ as a function of the average of $P\left(H^{+}\right)$and $P\left(H^{-}\right)$, assuming $\epsilon_{S}=.2$ and $\epsilon_{M}=.3$.

Figure EC.2A illustrates that $P(H)$ is regressive as a function of the average of $P\left(H^{+}\right)$and $P\left(H^{-}\right)$.

EC.2.2.2. Double Matching Violation Rates To show that the frequency of double matching violations of the two types depends on the frequency of the various types, we compare

$$
P\left(H L^{+} L^{-}\right)=p_{H H}\left(1-\epsilon_{M}\right)\left(\epsilon_{S}^{2}\right)+\frac{1}{2} p_{H L}(\epsilon)\left(1-\epsilon_{S}\right)+\frac{1}{2} p_{L H}\left(\epsilon_{S}\right)\left(1-\epsilon_{S}\right)+p_{L L}\left(\epsilon_{M}\right)\left(1-\epsilon_{S}\right)^{2}
$$

with

$$
P\left(L H^{+} H^{-}\right)=p_{H H}\left(\epsilon_{M}\right)\left(1-\epsilon_{S}\right)^{2}+\frac{1}{2} p_{H L}\left(\epsilon_{S}\right)\left(1-\epsilon_{S}\right)+\frac{1}{2} p_{L H}\left(\epsilon_{S}\right)\left(1-\epsilon_{S}\right)+p_{L L}\left(1-\epsilon_{M}\right)\left(\epsilon_{S}^{2}\right) .
$$

Simplifying $P\left(H L^{+} L^{-}\right)>P\left(L H^{+} H^{-}\right)$, we get $\left(p_{H H}-p_{L L}\right)\left(\epsilon_{S}^{2}\left(1-2 \epsilon_{M}\right)-\epsilon_{M}\left(1-2 \epsilon_{S}\right)\right)>0$. Note that if $\epsilon_{S}<\epsilon_{M}$, then $\epsilon_{S}^{2}\left(1-2 \epsilon_{M}\right)-\epsilon_{M}\left(1-2 \epsilon_{S}\right)<0$, since $\epsilon_{S}^{2}<\epsilon_{M}$. Thus, $P\left(L H^{+} H^{-}\right)>P\left(H L^{+} L^{-}\right)$ if $p_{H H}<p_{L L}$ and $P\left(H L^{+} L^{-}\right)>P\left(L H^{+} H^{-}\right)$if $p_{H H}>p_{L L}$.

Figure EC.2B plots the rates of double matching violations of the two types as a function of $p_{H H}$, assuming $\epsilon_{S}=.2$ and $\epsilon_{M}=.3$.

EC.2.2.3. Error rates: $P\left(H \mid L^{+} L^{-}\right)$and $P\left(L \mid H^{+} H^{-}\right)$The analysis of the error rates, $P\left(H \mid L^{+} L^{-}\right)$and $P\left(L \mid H^{+} H^{-}\right)$, under the mixed model is nearly identical to the analysis under the null model. The only change is that a different error rate applies to mixed gambles. Thus,

$$
P\left(H \mid L^{+} L^{-}\right)=\frac{p_{H H}\left(1-\epsilon_{M}\right)\left(\epsilon_{S}^{2}\right)+\frac{1}{2} p_{H L}\left(\epsilon_{S}\right)\left(1-\epsilon_{S}\right)+\frac{1}{2} p_{L H}\left(1-\epsilon_{S}\right)\left(\epsilon_{S}\right)+p_{L L}\left(\epsilon_{M}\right)\left(1-\epsilon_{S}\right)^{2}}{p_{H H}\left(\epsilon_{S}^{2}\right)+p_{H L}\left(\epsilon_{S}\right)\left(1-\epsilon_{S}\right)+p_{L H}\left(1-\epsilon_{S}\right)\left(\epsilon_{S}\right)+p_{L L}\left(1-\epsilon_{S}\right)^{2}},
$$

and

$$
P\left(L \mid H^{+} H^{-}\right)=\frac{p_{H H}\left(\epsilon_{M}\right)\left(1-\epsilon_{S}\right)^{2}+\frac{1}{2} p_{H L}\left(\epsilon_{S}\right)\left(1-\epsilon_{S}\right)+\frac{1}{2} p_{L H}\left(1-\epsilon_{S}\right)\left(\epsilon_{S}\right)+p_{L L}\left(1-\epsilon_{M}\right)\left(\epsilon_{S}^{2}\right)}{p_{H H}\left(1-\epsilon_{S}\right)^{2}+p_{H L}\left(\epsilon_{S}\right)\left(1-\epsilon_{S}\right)+p_{L H}\left(1-\epsilon_{S}\right)\left(\epsilon_{S}\right)+p_{L L}\left(\epsilon_{S}^{2}\right)} .
$$

Simplifying $P\left(H \mid L^{+} L^{-}\right)>P\left(L \mid H^{+} H^{-}\right)$, we get

$$
\begin{aligned}
& \left(p_{H H}-p_{L L}\right)\left(\epsilon_{S}\right)\left(1-\epsilon_{S}\right) \times \\
& \left(\left(p_{H H}+p_{L L}\right)\left(1-2 \epsilon_{M}\right)\left(1-\epsilon_{S}\right)\left(\epsilon_{S}\right)+\left(p_{H L}+p_{L H}\right)\left[\frac{1}{2}\left(1-2 \epsilon_{S}\right)+\left(1-\epsilon_{M}\right)\left(\epsilon_{S}^{2}\right)-\epsilon_{M}\left(1-\epsilon_{S}\right)^{2}\right]\right)>0,
\end{aligned}
$$

where $\frac{1}{2}\left(1-2 \epsilon_{S}\right)+\left(1-\epsilon_{M}\right)\left(\epsilon_{S}^{2}\right)-\epsilon_{M}\left(1-\epsilon_{S}\right)^{2} \geq 0$ if $\epsilon_{M}<.5$ and $\epsilon_{S}<.5$. Thus, $P\left(H \mid L^{+} L^{-}\right)>$ $P\left(L \mid H^{+} H^{-}\right)$if $p_{H H}>p_{L L}$, with the reverse holding if $p_{H H}<p_{L L}$.

Figure EC.2C plots $P\left(H \mid L^{+} L^{-}\right)$and $P\left(L \mid H^{+} H^{-}\right)$as a function of $p_{H H}$, assuming $\epsilon_{S}=.2$ and $\epsilon_{M}=.3$. 
Figure EC.2 Implications of mixed error model on choice percentages, double matching violation rates, error rates, and conditional probabilities.
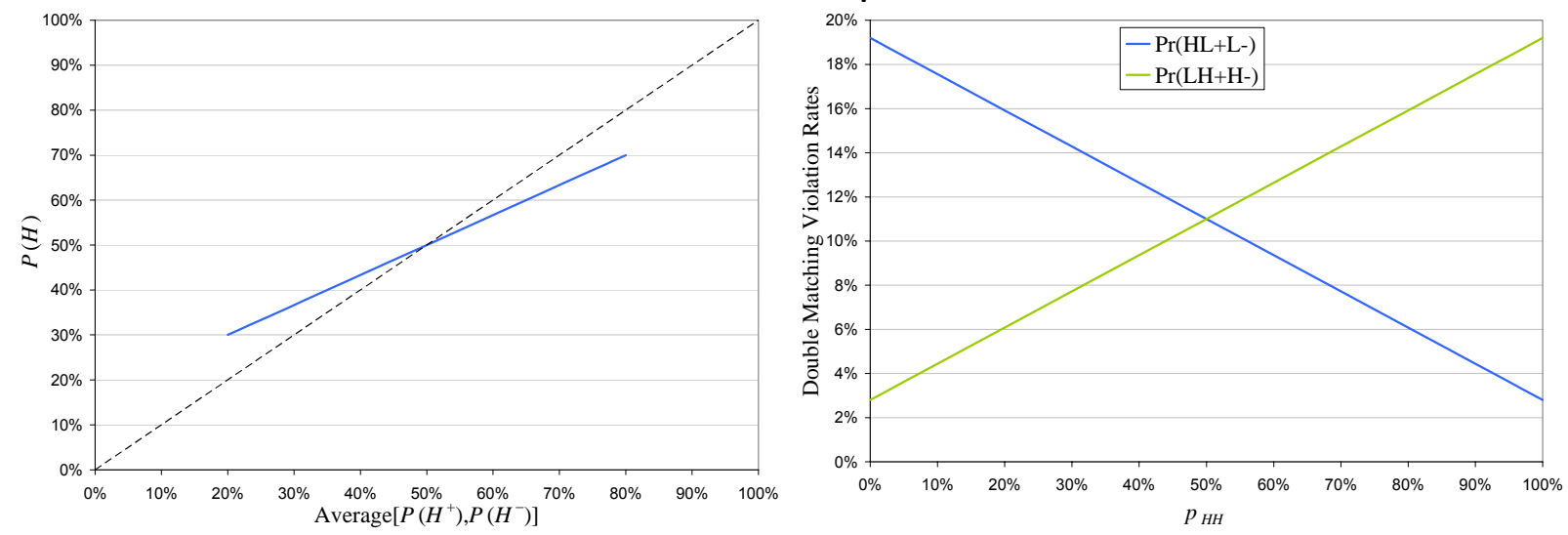

A: Choice Percentages

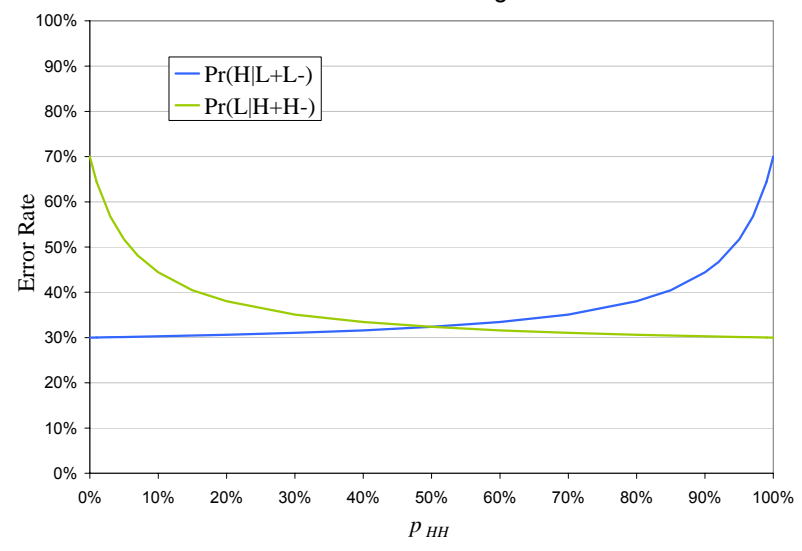

C: Error Rates

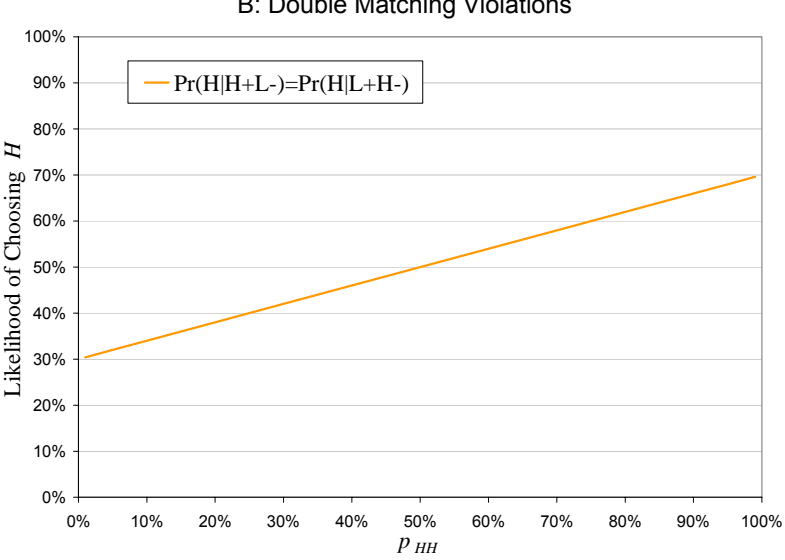

D: Conditional Probabilities

Note. To simplify the presentation, we assume that $p_{H L}=p_{L H}=0$ and $\epsilon_{S}=.2$ and $\epsilon_{M}=\epsilon_{H}=\epsilon_{L}=.3$. Panel A illustrates choice percentages, $P(H)$, as a function of the average of $P\left(H^{+}\right)$and $P\left(H^{-}\right)$. The mixed error model requires that $P(H)$ exceed $\frac{P\left(H^{+}\right)+P\left(H^{-}\right)}{2}$ if $p_{H H}<p_{L L}$, but the reverse hold for $p_{H H}>p_{L L}$. Panel B depicts double matching violation rates, $P\left(H L^{+} L^{-}\right)$and $P\left(L H^{+} H^{-}\right)$, as a function of $p_{H H}$, the probability of type $\theta_{H^{+} H^{-}}$. The mixed error model requires that double matching violations $H L^{+} L^{-}$exceed double matching violations $L H^{+} H^{-}$if $p_{H H}<p_{L L}$, but that the reverse holds for $p_{H H}>p_{L L}$. Panel $\mathrm{C}$ shows error rates, $P\left(H \mid L^{+} L^{-}\right)$and $P\left(L \mid H^{+} H^{-}\right)$, as a function of the frequency of $p_{H H}$. The mixed error model requires that error rate $P\left(L \mid H^{+} H^{-}\right)$exceed $P\left(H \mid L^{+} L^{-}\right)$ for $p_{H H}<p_{L L}$, with the reverse holding for $p_{H H}>p_{L L}$. Panel D illustrates conditional probabilities, $P\left(H \mid H^{+} L^{-}\right)$ and $P\left(H \mid L^{+} H^{-}\right)$as a function of $p_{H H}$. The mixed error model requires that the likelihood of choosing $H$ given one of the indeterminate patterns, $H^{+} L^{-}$and $L^{+} H^{-}$, be less than $\frac{1}{2}$ if $p_{H H}<p_{L L}$ and greater than $\frac{1}{2}$ if $p_{H H}>p_{L L}$.

EC.2.2.4. Likelihood of choosing $H$ for "indeterminate" patterns The logic of deriving restrictions on the likelihood of choosing $H$ given the "indeterminate" patterns, $H^{+} L^{-}$and $L^{+} H^{-}$, is identical under the mixed error model as under the null error model. Since

$$
P\left(H \mid H^{+} L^{-}\right)=\frac{p_{H H}\left(1-\epsilon_{M}\right)\left(1-\epsilon_{S}\right)\left(\epsilon_{S}\right)+\frac{1}{2} p_{H L}\left(1-\epsilon_{S}\right)^{2}+\frac{1}{2} p_{L H}\left(\epsilon_{S}^{2}\right)+p_{L L}\left(\epsilon_{M}\right)\left(\epsilon_{S}\right)\left(1-\epsilon_{S}\right)}{p_{H H}\left(1-\epsilon_{S}\right)\left(\epsilon_{S}\right)+p_{H L}\left(1-\epsilon_{S}\right)^{2}+p_{L H}\left(\epsilon_{S}^{2}\right)+p_{L L}\left(1-\epsilon_{S}\right)\left(\epsilon_{S}\right)},
$$

$P\left(H \mid H^{+} L^{-}\right)>($resp. $<) \frac{1}{2}$ if $\left(p_{H H}-p_{L L}\right)\left(1-\epsilon_{S}\right)\left(\epsilon_{S}\right)\left(1-2 \epsilon_{M}\right)>($ resp. $<) 0$, which holds if $\epsilon_{S}<.5$, $\epsilon_{M}<.5$ and $p_{H H}>($ resp. $<) p_{L L}$. A similar manipulation shows that $P\left(H \mid L^{+} H^{-}\right)>($resp. $<) \frac{1}{2}$ if $\epsilon_{S}<.5$ and $p_{H H}>($ resp. $<) p_{L L}$.

Figure EC.2D plots $P\left(H \mid H^{+} L^{-}\right)$and $P\left(H \mid L^{+} H^{-}\right)$as a function of $p_{H H}$, assuming $\epsilon_{S}=.2$ and $\epsilon_{M}=.3$. 


\section{EC.2.3. Implications of asymmetric model}

We next show that the asymmetric error model has different implications on the measures considered than either the null error model or the mixed error model. The null and mixed error models all require a symmetry around $p_{H H}=p_{L L}$, whereas the asymmetric error model does not.

Recall that Problems 1 through 3 suggested a process in which decision makers were less sensitive to probability differences when choosing among mixed gambles than when choosing among singledomain gambles. This process applied to our gambles suggests that a greater tendency to choose $H$ over $L$, than $H^{+}$over $L^{+}$or $H^{-}$over $L^{-}$. The asymmetric error model captures this choice pattern by requiring that the error rate for $\theta_{L^{+} L^{-}}$types choosing $H$ be higher the other error rates: $\epsilon_{H}>\epsilon_{S}=\epsilon_{L}$. Throughout this section, we let $\epsilon_{\bar{H}}=\epsilon_{S}=\epsilon_{L}$.

EC.2.3.1. Choice percentages We begin by considering the implications of the asymmetric error model for the choice percentages, $P(H), P\left(H^{+}\right)$, and $P\left(H^{-}\right): P\left(H^{+}\right)=\left(p_{H H}+p_{H L}\right)(1-$ $\left.\epsilon_{\bar{H}}\right)+\left(p_{L H}+p_{L L}\right)\left(\epsilon_{\bar{H}}\right), P\left(H^{-}\right)=\left(p_{H H}+p_{L H}\right)\left(1-\epsilon_{\bar{H}}\right)+\left(p_{H L}+p_{L L}\right)\left(\epsilon_{\bar{H}}\right)$, and $P(H)=\left(p_{H H}\right)(1-$ $\left.\epsilon_{\bar{H}}\right)+\frac{1}{2}\left(1-\left(\epsilon_{\bar{H}}-\epsilon_{H}\right)\right)\left(p_{H L}+p_{L H}\right)+\left(p_{L L}\right)\left(\epsilon_{H}\right)$. Then, $P(H)>\frac{P\left(H^{+}\right)+P\left(H^{-}\right)}{2}$ if $\left(p_{H H}\right)\left(1-\epsilon_{\bar{H}}\right)+$ $\frac{1}{2}\left(1-\left(\epsilon_{\bar{H}}-\epsilon_{H}\right)\right)\left(p_{H L}+p_{L H}\right)+\left(p_{L L}\right)\left(\epsilon_{H}\right)>\left(p_{H H}\right)\left(1-\epsilon_{\bar{H}}\right)+\frac{1}{2}\left(p_{H L}+p_{L H}\right)+\left(p_{L L}\right)\left(\epsilon_{\bar{H}}\right)$, or $\left(\epsilon_{H}-\right.$ $\left.\epsilon_{\bar{H}}\right)\left[\frac{1}{2}\left(p_{H L}+p_{L H}\right)+p_{L L}\right]>0$, which holds if $\epsilon_{H}>\epsilon_{\bar{H}}$.

Figure EC.3A plots $P(H)$ as a function of the average of $P\left(H^{+}\right)$and $P\left(H^{-}\right)$, assuming $\epsilon_{\bar{H}}=.2$ and $\epsilon_{H}=.3$.

EC.2.3.2. Double Matching Violation Rates We next consider the implications of the asymmetric error model on the frequency of double matching violations. We compare the probabilities of the two types of double matching violations,

$$
\begin{array}{r}
P\left(H L^{+} L^{-}\right)=p_{H H}\left(1-\epsilon_{\bar{H}}\right)\left(\epsilon_{\bar{H}}^{2}\right)+\frac{1}{2} p_{H L}\left(1-\left(\epsilon_{\bar{H}}-\epsilon_{H}\right)\right)\left(\epsilon_{\bar{H}}\right)\left(1-\epsilon_{\bar{H}}\right)+ \\
\frac{1}{2} p_{L H}\left(1-\left(\epsilon_{\bar{H}}-\epsilon_{H}\right)\right)\left(\epsilon_{\bar{H}}\right)\left(1-\epsilon_{\bar{H}}\right)+p_{L L}\left(1-\epsilon_{\bar{H}}\right)^{2}\left(\epsilon_{H}\right)
\end{array}
$$

and

$$
\begin{array}{r}
P\left(L H^{+} H^{-}\right)=p_{H H}\left(1-\epsilon_{\bar{H}}\right)^{2}\left(\epsilon_{\bar{H}}\right)+\frac{1}{2} p_{H L}\left(1-\left(\epsilon_{H}-\epsilon_{\bar{H}}\right)\right)\left(\epsilon_{\bar{H}}\right)\left(1-\epsilon_{\bar{H}}\right)+ \\
\frac{1}{2} p_{L H}\left(1-\left(\epsilon_{H}-\epsilon_{\bar{H}}\right)\right)\left(\epsilon_{\bar{H}}\right)\left(1-\epsilon_{\bar{H}}\right)+p_{L L}\left(1-\epsilon_{H}\right)\left(\epsilon_{\bar{H}}^{2}\right) .
\end{array}
$$

Then, $P\left(H L^{+} L^{-}\right)>P\left(L H^{+} H^{-}\right)$if

$$
\begin{array}{r}
p_{H H}\left(1-\epsilon_{\bar{H}}\right)\left(\epsilon_{\bar{H}}\right)\left(2 \epsilon_{\bar{H}}-1\right)+\frac{1}{2} p_{H L}\left(2 \epsilon_{H}-2 \epsilon_{\bar{H}}\right)\left(\epsilon_{\bar{H}}\right)\left(1-\epsilon_{\bar{H}}\right)+ \\
\frac{1}{2} p_{L H}\left(2 \epsilon_{H}-2 \epsilon_{\bar{H}}\right)\left(\epsilon_{\bar{H}}\right)\left(1-\epsilon_{\bar{H}}\right)+p_{L L}\left[\left(1-\epsilon_{\bar{H}}\right)^{2}\left(\epsilon_{H}\right)-\left(1-\epsilon_{H}\right)\left(\epsilon_{\bar{H}}^{2}\right)\right]>0 .
\end{array}
$$

Letting $p_{H H}=p_{L L}$, we get

$p_{H H}\left[\left(1-\epsilon_{\bar{H}}\right)\left(\epsilon_{\bar{H}}\right)\left(2 \epsilon_{\bar{H}}-1\right)+\left(1-\epsilon_{\bar{H}}\right)^{2}\left(\epsilon_{H}\right)-\left(1-\epsilon_{H}\right)\left(\epsilon_{\bar{H}}^{2}\right)\right]+\frac{1}{2}\left(p_{H L}+p_{L H}\right)\left(2 \epsilon_{H}-2 \epsilon_{\bar{H}}\right)\left(\epsilon_{\bar{H}}\right)\left(1-\epsilon_{\bar{H}}\right)>0$,

or

$$
p_{H H}\left[\left(\epsilon_{H}-\epsilon_{\bar{H}}\right)\left(2 \epsilon_{\bar{H}}^{2}-2 \epsilon_{\bar{H}}+1\right)\right]+\frac{1}{2}\left(p_{H L}+p_{L H}\right)\left(2 \epsilon_{H}-2 \epsilon_{\bar{H}}\right)\left(\epsilon_{\bar{H}}\right)\left(1-\epsilon_{\bar{H}}\right)>0,
$$

which holds if $\epsilon_{H}>\epsilon_{\bar{H}}$. To show that $P\left(H L^{+} L^{-}\right)>P\left(L H^{+} H^{-}\right)$if $p_{H H}>p_{L L}$, we substitute $p_{L L}=$ $1-p_{H H}-p_{H L}-p_{L H}$. It suffices to show that

$$
\frac{d P\left(H L^{+} L^{-}\right)}{d p_{H H}}-\frac{P\left(L H^{+} H^{-}\right)}{d p_{H H}}=\left(\epsilon_{H}-\epsilon_{\bar{H}}\right)\left[\left(1-\epsilon_{\bar{H}}\right)^{2}+\epsilon_{\bar{H}}^{2}\right]>0,
$$

which holds for $\epsilon_{H}>\epsilon_{\bar{H}}$.

Figure EC.3B plots the rates of double matching violations of the two types as a function of $p_{H H}$, assuming $\epsilon_{\bar{H}}=.2$ and $\epsilon_{H}=.3$. 
Figure EC.3 Implications of asymmetric error model on choice percentages, double matching violation rates, error rates, and conditional probabilities.
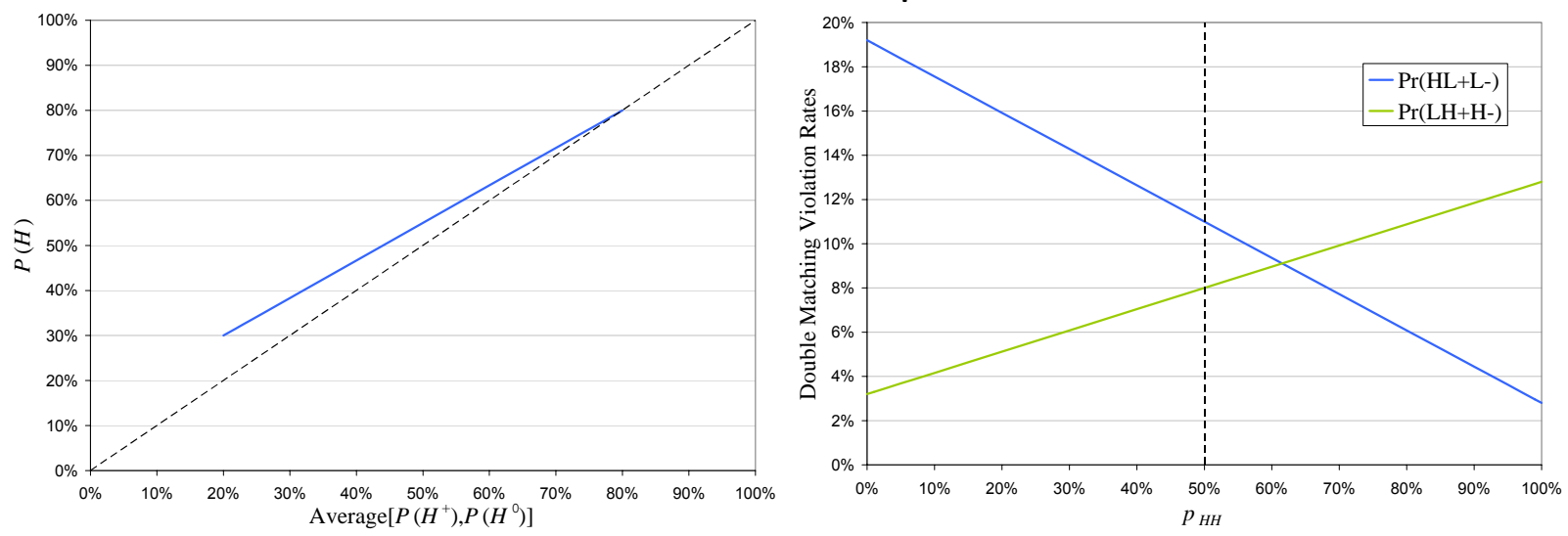

A: Choice Percentages

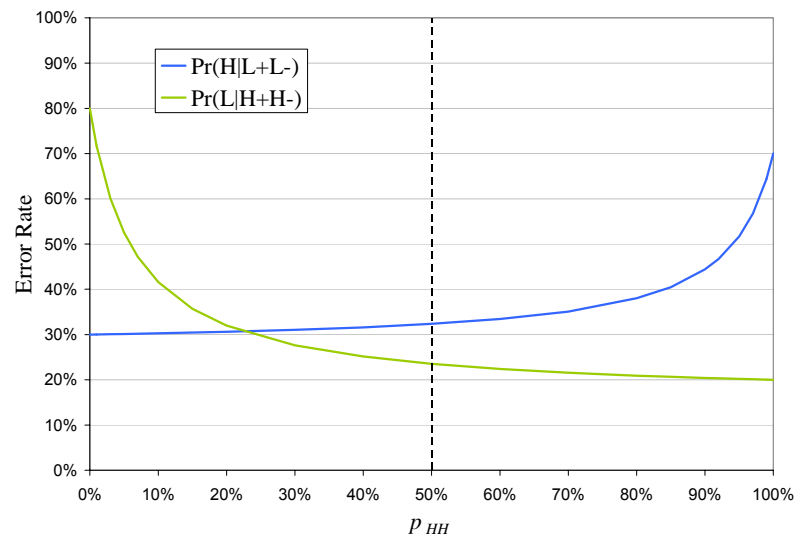

C: Error Rates

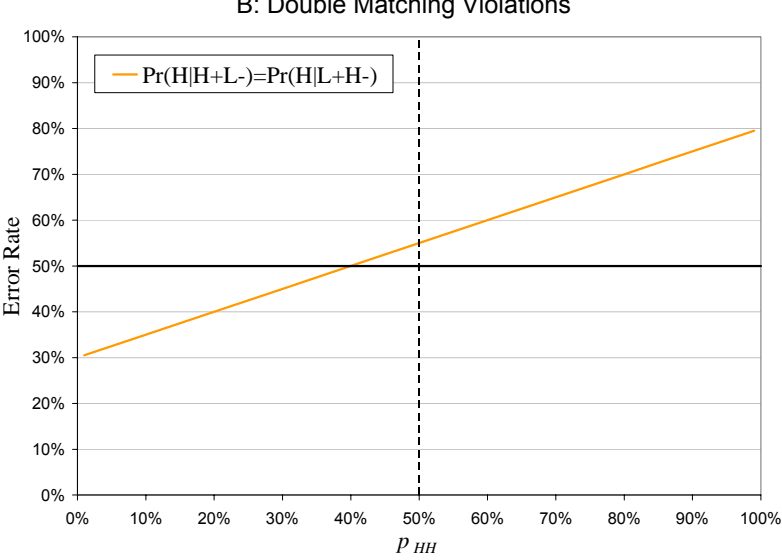

D: Conditional Probabilities

Note. To simplify the presentation, we assume that $p_{H L}=p_{L H}=0$ and $\epsilon_{S}=\epsilon_{L}=.2$ and $\epsilon_{H}=.3$. Panel A illustrates choice percentages, $P(H)$, as a function of the average of $P\left(H^{+}\right)$and $P\left(H^{-}\right)$. The asymmetric error model requires $P(H)$ exceed $\frac{P\left(H^{+}\right)+P\left(H^{-}\right)}{2}$ for all $p_{H H}$. Panel B depicts double matching violation rates, $P\left(H L^{+} L^{-}\right)$and $P\left(L H^{+} H^{-}\right)$, as a function of $p_{H H}$, the probability of type $\theta_{H^{+} H^{-}}$. The asymmetric error model implies an asymmetry in double matching violations. Double matching violation $H L^{+} L^{-}$is more prevalent than double matching violations $L H^{+} H^{-}$for $p_{H H}=p_{L L}$, with the reverse holding for some $p_{H H}$ larger than $p_{L L}$. Panel $\mathrm{C}$ shows error rates, $P\left(H \mid L^{+} L^{-}\right)$and $P\left(L \mid H^{+} H^{-}\right)$, as a function of the frequency of $p_{H H}$. The asymmetric error model implies an asymmetry in error rates: error rate, $P\left(H \mid L^{+} L^{-}\right)$, exceeds error rate, $P\left(L \mid H^{+} H^{-}\right)$, for $p_{H H}=p_{L L}$, with the reverse holding for some $p_{H H}$ smaller than $p_{L L}$. Panel D illustrates conditional probabilities, $P\left(H \mid H^{+} L^{-}\right)$and $P\left(H \mid L^{+} H^{-}\right)$ as a function of $p_{H H}$. The asymmetric error model implies an asymmetry in the likelihood of choosing $H$ given an indeterminate pattern: conditional probabilities $P\left(H \mid H^{+} L^{-}\right)$and $P\left(H \mid L^{+} H^{-}\right)$exceed $\frac{1}{2}$ for $p_{H H}=p_{L L}$, with the reverse holding for some $p_{H H}$ smaller than $p_{L L}$.

EC.2.3.3. Error rates: $P\left(H \mid L^{+} L^{-}\right)$and $P\left(L \mid H^{+} H^{-}\right)$Note that $P\left(H \mid \theta_{H^{+} L^{-}}\right)=$ $\frac{1}{2} P\left(H \mid \theta_{H^{+} H^{-}}\right)+\frac{1}{2} P\left(H \mid \theta_{L^{+} L^{-}}\right)=\frac{1}{2}\left(1-\epsilon_{\bar{H}}\right)+\frac{1}{2}\left(\epsilon_{H}\right)=\frac{1}{2}\left(1-\left(\epsilon_{\bar{H}}-\epsilon_{H}\right)\right)$. Similarly, $P\left(L \mid \theta_{H^{+} L^{-}}\right)=$ $\frac{1}{2} P\left(L \mid \theta_{H^{+} H^{-}}\right)+\frac{1}{2} P\left(L \mid \theta_{L^{+} L^{-}}\right)=\frac{1}{2}\left(\epsilon_{\bar{H}}\right)+\frac{1}{2}\left(1-\epsilon_{H}\right)=\frac{1}{2}\left(1-\left(\epsilon_{H}-\epsilon_{\bar{H}}\right)\right)$. Therefore,

$$
P\left(H \mid L^{+} L^{-}\right)=\frac{p_{H H}\left(1-\epsilon_{\bar{H}}\right)\left(\epsilon_{\bar{H}}^{2}\right)+\frac{1}{2}\left(1-\left(\epsilon_{\bar{H}}-\epsilon_{H}\right)\right)\left(p_{H L}+p_{L H}\right)\left(1-\epsilon_{\bar{H}}\right)\left(\epsilon_{\bar{H}}\right)+p_{L L}\left(\epsilon_{H}\right)\left(1-\epsilon_{\bar{H}}\right)^{2}}{p_{H}\left(\epsilon_{\bar{H}}^{2}\right)+p_{H L}\left(\epsilon_{\bar{H}}\right)\left(1-\epsilon_{\bar{H}}\right)+p_{L H}\left(1-\epsilon_{\bar{H}}\right)\left(\epsilon_{\bar{H}}\right)+p_{L L}\left(1-\epsilon_{\bar{H}}\right)^{2}},
$$

and

$$
P\left(L \mid H^{+} H^{-}\right)=\frac{p_{H H}\left(\epsilon_{\bar{H}}\right)\left(1-\epsilon_{\bar{H}}\right)^{2}+\frac{1}{2}\left(1-\left(\epsilon_{H}-\epsilon_{\bar{H}}\right)\right)\left(p_{H L}+p_{L H}\right)+p_{L L}\left(1-\epsilon_{H}\right)\left(\epsilon_{\bar{H}}^{2}\right)}{p_{H H}\left(1-\epsilon_{\bar{H}}\right)^{2}+p_{H L}\left(\epsilon_{\bar{H}}\right)\left(1-\epsilon_{\bar{H}}\right)+p_{L H}\left(1-\epsilon_{\bar{H}}\right)\left(\epsilon_{\bar{H}}\right)+p_{L L}\left(\epsilon_{\bar{H}}^{2}\right)} .
$$


We show that $P\left(H \mid L^{+} L^{-}\right)>P\left(L \mid H^{+} H^{-}\right)$if $p_{H H}=p_{L L}$. Letting $p_{H H}=p_{L L}$, we simplify $P\left(H \mid L^{+} L^{-}\right)>P\left(L \mid H^{+} H^{-}\right)$to get:

$$
\begin{aligned}
& \left(p_{H H}{ }^{2}\right)\left[\left(1-\epsilon_{\bar{H}}\right)^{2}\left(\epsilon_{\bar{H}}\right)^{2}\left(2 \epsilon_{H}-2 \epsilon_{\bar{H}}\right)+\left(1-\epsilon_{\bar{H}}\right)\left(\epsilon_{\bar{H}}\right)\left(\epsilon_{\bar{H}}^{3}-\left(1-\epsilon_{\bar{H}}\right)^{3}\right)+\left(\epsilon_{H}\right)\left(1-\epsilon_{\bar{H}}\right)^{4}-\left(1-\epsilon_{H}\right)\left(\epsilon_{\bar{H}}^{4}\right)\right] \\
+ & \left(p_{H L}+p_{L H}\right)\left(p_{H H}\right)\left(1-\epsilon_{\bar{H}}\right)\left(\epsilon_{\bar{H}}\right)\left[\left(1-\epsilon_{\bar{H}}\right)\left(\epsilon_{\bar{H}}\right)\left(2 \epsilon_{\bar{H}}-1\right)+\left(\epsilon_{H}\right)\left(1-\epsilon_{\bar{H}}\right)^{2}-\left(1-\epsilon_{H}\right)\left(\epsilon_{\bar{H}}^{2}\right)\right] \\
+ & \left(\frac{1}{2}\right)\left(2 \epsilon_{H}-2 \epsilon_{\bar{H}}\right)\left[\left(p_{H L}+p_{L H}\right)\left(p_{H H}\right)\left(\left(1-\epsilon_{\bar{H}}\right)^{3}\left(\epsilon_{\bar{H}}\right)^{1}+\left(1-\epsilon_{\bar{H}}\right)^{1}\left(\epsilon_{\bar{H}}\right)^{3}\right)+\left(p_{H L}+p_{L H}\right)^{2}\left(1-\epsilon_{\bar{H}}\right)^{2}\left(\epsilon_{\bar{H}}\right)^{2}\right]>0,
\end{aligned}
$$

which after manipulation yields,

$$
\begin{gathered}
\left(p_{H H}^{2}\right)\left(\epsilon_{H}-\epsilon_{\bar{H}}\right)\left(2 \epsilon_{\bar{H}}^{2}-2 \epsilon_{\bar{H}}+1\right)^{2} \\
+\quad\left(p_{H L}+p_{L H}\right)\left(p_{H H}\right)\left(1-\epsilon_{\bar{H}}\right)\left(\epsilon_{\bar{H}}\right)\left(\epsilon_{H}-\epsilon_{\bar{H}}\right)\left(2 \epsilon_{\bar{H}}^{2}-2 \epsilon_{\bar{H}}+1\right) \\
+\left(\frac{1}{2}\right)\left(2 \epsilon_{H}-2 \epsilon_{\bar{H}}\right)\left[\left(p_{H L}+p_{L H}\right)\left(p_{H H}\right)\left(\left(1-\epsilon_{\bar{H}}\right)^{3}\left(\epsilon_{\bar{H}}\right)^{1}+\left(1-\epsilon_{\bar{H}}\right)^{1}\left(\epsilon_{\bar{H}}\right)^{3}\right)+\left(p_{H L}+p_{L H}\right)^{2}\left(1-\epsilon_{\bar{H}}\right)^{2}\left(\epsilon_{\bar{H}}\right)^{2}\right]>0 .
\end{gathered}
$$

which holds if $\epsilon_{H}>\epsilon_{\bar{H}}$ (since $2 \epsilon_{\bar{H}}^{2}-2 \epsilon_{\bar{H}}+1>0$ if $\epsilon_{\bar{H}}<.5$ ).

Figure EC.3C plots $P\left(H \mid L^{+} L^{-}\right)$and $P\left(L \mid H^{+} H^{-}\right)$as a function of $p_{H H}$, assuming $\epsilon_{\bar{H}}=.2$ and $\epsilon_{H}=.3$.

EC.2.3.4. Likelihood of choosing $H$ for "indeterminate" patterns Finally, we derive restrictions on the likelihood of choosing $H$ given the "indeterminate" patterns, $H^{+} L^{-}$and $L^{+} H^{-}$. Since

$$
P\left(H \mid H^{+} L^{-}\right)=\frac{p_{H H}\left(1-\epsilon_{\bar{H}}\right)^{2}\left(\epsilon_{\bar{H}}\right)+\frac{1}{2} p_{H L}\left(1-\left(\epsilon_{\bar{H}}-\epsilon_{H}\right)\right)\left(1-\epsilon_{\bar{H}}\right)^{2}+\frac{1}{2} p_{L H}\left(1-\left(\epsilon_{\bar{H}}-\epsilon_{H}\right)\right)\left(\epsilon_{\bar{H}}^{2}\right)+p_{L L}\left(\epsilon_{H}\right)\left(\epsilon_{\bar{H}}\right)(1-}{p_{H H}\left(1-\epsilon_{\bar{H}}\right)\left(\epsilon_{\bar{H}}\right)+p_{H L}\left(1-\epsilon_{\bar{H}}\right)^{2}+p_{L H}\left(\epsilon_{\bar{H}}^{2}\right)+p_{L L}\left(\epsilon_{\bar{H}}\right)\left(1-\epsilon_{\bar{H}}\right)}
$$

$P\left(H \mid H^{+} L^{-}\right)>\frac{1}{2}$ if

$p_{H H}\left(1-\epsilon_{\bar{H}}\right)\left(\epsilon_{\bar{H}}\right)\left(1-2 \epsilon_{\bar{H}}\right)+p_{H L}\left(\epsilon_{H}-\epsilon_{\bar{H}}\right)\left(1-\epsilon_{\bar{H}}\right)^{2}+p_{L H}\left(\epsilon_{H}-\epsilon_{\bar{H}}\right)\left(\epsilon_{\bar{H}}^{2}\right)+p_{L L}\left(2 \epsilon_{H}-1\right)\left(\epsilon_{\bar{H}}\right)\left(1-\epsilon_{\bar{H}}\right)>0$.

To show that $P\left(H \mid H^{+} L^{-}\right)>\frac{1}{2}$ if $p_{H H}=p_{L L}$, we substitute $p_{H H}=p_{L L}$ in the previous expression:

$$
p_{H H}\left(1-\epsilon_{\bar{H}}\right)\left(\epsilon_{\bar{H}}\right)\left(2 \epsilon_{H}-2 \epsilon_{\bar{H}}\right)+p_{H L}\left(\epsilon_{H}-\epsilon_{\bar{H}}\right)\left(1-\epsilon_{\bar{H}}\right)^{2}+p_{L H}\left(\epsilon_{H}-\epsilon_{\bar{H}}\right)\left(\epsilon_{\bar{H}}^{2}\right)>0,
$$

which holds if $\epsilon_{H}>\epsilon_{\bar{H}}$. A similar manipulation shows that $P\left(H \mid L^{+} H^{-}\right)>\frac{1}{2}$ if $p_{H H}=p_{L L}$.

Figure EC.3D plots $P\left(H \mid H^{+} L^{-}\right)$and $P\left(H \mid L^{+} H^{-}\right)$as a function of $p_{H H}$, assuming $\epsilon_{\bar{H}}=.2$ and $\epsilon_{H}=.3$.

\section{EC.3. Empirical Data and Implications of Error Models for Study 1}

In this section, we analyze the empirical data from Study 1 of $\mathrm{Wu}$ and Markle (2007) with respect to the four implications derived in the last section for each of the three error models. Most of the empirical implications from the previous section were functions of the relative frequency of types $\theta_{H^{+} H^{-}}$and $\theta_{L^{+} L^{-}}$. Of course, the frequency of types is not observable. Therefore, we present the empirical data as a function of the average of $\% H^{+}$and $\% H^{-}$, the percentage of participants choosing $H^{+}$over $L^{-}$, and $H^{-}$over $L^{-}$, respectively. To show that this analysis is equivalent in expectation, we need to show that $p_{H H}>p_{L L}$ if and only if $\frac{P\left(H^{+}\right)+P\left(H^{-}\right)}{2}>\frac{1}{2}$. Noting that $\frac{P\left(H^{+}\right)+P\left(H^{-}\right)}{2}=p_{H H}\left(1-\epsilon_{S}\right)+\frac{1}{2}\left(p_{H L}+p_{L H}\right)+p_{L L}\left(\epsilon_{S}\right)$ for all 3 error models, we substitute $p_{H L}+$ $p_{L H}=1-p_{H H}-p_{L L}$ to get $\frac{P\left(H^{+}\right)+P\left(H^{-}\right)}{2}=\frac{1}{2}+\left(p_{H H}-p_{L L}\right)\left(\frac{1}{2}-\epsilon_{S}\right)$, which exceeds $\frac{1}{2}$ if and only if $p_{H H}>p_{L L}$.

The four measures of double matching violations are shown in Table EC.3. The first three measures are repeated from $\mathrm{Wu}$ and Markle. The measures for the likelihood of choosing $H$ for indeterminate patterns is not found in the main text. 
Table EC.3 Measures of double matching violations for 34 tests of double matching (Study 1)

\begin{tabular}{|c|c|c|c|c|c|c|c|c|c|c|}
\hline \multirow[b]{2}{*}{ Test } & \multicolumn{4}{|c|}{ Choice Percentages } & \multicolumn{2}{|c|}{ Double Matching Violations } & \multicolumn{2}{|c|}{$\begin{array}{l}\text { Double Matching } \\
\text { Error Rates }\end{array}$} & \multicolumn{2}{|c|}{$\begin{array}{l}\text { Likelihood of Choosing } H \\
\text { for indeterminate patterns }\end{array}$} \\
\hline & $\% H$ & $\% H^{+}$ & $\% H^{-}$ & $\frac{\% H^{+}+\% H^{-}}{2}$ & $\% H L^{+} L^{-}$ & $\% L H^{+} H^{-}$ & $P\left(H \mid L^{+} L^{-}\right)$ & $P\left(L \mid H^{+} H^{-}\right)$ & $P\left(H \mid H^{+} L^{-}\right)$ & $P\left(H \mid L^{+} H^{-}\right)$ \\
\hline 1 & $22.2 \%$ & $9.9 \%$ & $17.3 \%$ & $13.6 \%$ & $12.3 \%$ & $1.2 \%$ & $16.4 \%$ & $50.0 \%$ & $50.0 \%$ & $33.3 \%$ \\
\hline 2 & $21.0 \%$ & $17.3 \%$ & $14.8 \%$ & $16.1 \%$ & $6.2 \%$ & $2.5 \%$ & $8.6 \%$ & $66.7 \%$ & $54.5 \%$ & $55.6 \%$ \\
\hline 3 & $28.3 \%$ & $11.9 \%$ & $20.3 \%$ & $16.1 \%$ & $11.9 \%$ & $0.0 \%$ & $16.7 \%$ & $0.0 \%$ & $40.0 \%$ & $60.0 \%$ \\
\hline 4 & $33.3 \%$ & $18.1 \%$ & $22.2 \%$ & $20.2 \%$ & $16.7 \%$ & $2.8 \%$ & $25.5 \%$ & $50.0 \%$ & $33.3 \%$ & $58.3 \%$ \\
\hline 5 & $43.1 \%$ & $20.8 \%$ & $25.0 \%$ & $22.9 \%$ & $25.0 \%$ & $6.9 \%$ & $37.5 \%$ & $55.6 \%$ & $83.3 \%$ & $44.4 \%$ \\
\hline 6 & $51.4 \%$ & $26.4 \%$ & $25.0 \%$ & $25.7 \%$ & $23.6 \%$ & $4.2 \%$ & $41.5 \%$ & $50.0 \%$ & $61.5 \%$ & $75.0 \%$ \\
\hline 7 & $51.9 \%$ & $14.8 \%$ & $37.0 \%$ & $25.9 \%$ & $28.4 \%$ & $2.5 \%$ & $50.0 \%$ & $28.6 \%$ & $20.0 \%$ & $56.5 \%$ \\
\hline 8 & $48.3 \%$ & $16.7 \%$ & $46.7 \%$ & $31.7 \%$ & $21.7 \%$ & $6.7 \%$ & $43.3 \%$ & $50.0 \%$ & $0.0 \%$ & $60.0 \%$ \\
\hline 9 & $58.3 \%$ & $16.7 \%$ & $55.0 \%$ & $35.9 \%$ & $18.3 \%$ & $3.3 \%$ & $45.8 \%$ & $28.6 \%$ & $33.3 \%$ & $69.2 \%$ \\
\hline 10 & $51.3 \%$ & $47.5 \%$ & $27.5 \%$ & $37.5 \%$ & $17.5 \%$ & $6.3 \%$ & $41.2 \%$ & $35.7 \%$ & $62.5 \%$ & $37.5 \%$ \\
\hline 12 & $59.3 \%$ & $42.0 \%$ & $35.8 \%$ & $38.9 \%$ & $16.0 \%$ & $2.5 \%$ & $43.3 \%$ & $16.7 \%$ & $77.3 \%$ & $47.1 \%$ \\
\hline 11 & $54.2 \%$ & $33.3 \%$ & $44.4 \%$ & $38.9 \%$ & $15.3 \%$ & $6.9 \%$ & $45.8 \%$ & $62.5 \%$ & $56.3 \%$ & $66.7 \%$ \\
\hline 13 & $51.7 \%$ & $38.3 \%$ & $41.7 \%$ & $40.0 \%$ & $11.7 \%$ & $5.0 \%$ & $30.4 \%$ & $27.3 \%$ & $66.7 \%$ & $57.1 \%$ \\
\hline 14 & $57.6 \%$ & $33.9 \%$ & $47.5 \%$ & $40.7 \%$ & $15.3 \%$ & $3.4 \%$ & $56.3 \%$ & $40.0 \%$ & $73.3 \%$ & $47.8 \%$ \\
\hline 15 & $51.3 \%$ & $51.3 \%$ & $32.5 \%$ & $41.9 \%$ & $10.0 \%$ & $5.0 \%$ & $29.6 \%$ & $28.6 \%$ & $59.3 \%$ & $58.3 \%$ \\
\hline 16 & $65.0 \%$ & $42.5 \%$ & $42.5 \%$ & $42.5 \%$ & $15.0 \%$ & $1.3 \%$ & $46.2 \%$ & $7.1 \%$ & $80.0 \%$ & $55.0 \%$ \\
\hline 17 & $58.8 \%$ & $47.5 \%$ & $41.3 \%$ & $44.4 \%$ & $16.3 \%$ & $8.8 \%$ & $44.8 \%$ & $35.0 \%$ & $61.1 \%$ & $76.9 \%$ \\
\hline 18 & $71.7 \%$ & $51.7 \%$ & $41.7 \%$ & $46.7 \%$ & $16.7 \%$ & $1.7 \%$ & $62.5 \%$ & $8.3 \%$ & $63.2 \%$ & $76.9 \%$ \\
\hline 19 & $57.5 \%$ & $55.0 \%$ & $43.8 \%$ & $49.4 \%$ & $11.3 \%$ & $5.0 \%$ & $42.9 \%$ & $20.0 \%$ & $58.3 \%$ & $46.7 \%$ \\
\hline 20 & $40.0 \%$ & $46.7 \%$ & $53.3 \%$ & $50.0 \%$ & $5.0 \%$ & $6.7 \%$ & $27.3 \%$ & $36.4 \%$ & $41.2 \%$ & $33.3 \%$ \\
\hline 21 & $71.3 \%$ & $58.8 \%$ & $47.5 \%$ & $53.2 \%$ & $11.3 \%$ & $1.3 \%$ & $45.0 \%$ & $4.0 \%$ & $77.3 \%$ & $53.8 \%$ \\
\hline 22 & $63.3 \%$ & $58.3 \%$ & $48.3 \%$ & $53.3 \%$ & $10.0 \%$ & $8.3 \%$ & $54.5 \%$ & $33.3 \%$ & $50.0 \%$ & $85.7 \%$ \\
\hline 23 & $70.0 \%$ & $58.8 \%$ & $50.0 \%$ & $54.4 \%$ & $17.5 \%$ & $5.0 \%$ & $63.6 \%$ & $13.8 \%$ & $55.6 \%$ & $63.6 \%$ \\
\hline 24 & $78.8 \%$ & $53.8 \%$ & $55.0 \%$ & $54.4 \%$ & $18.8 \%$ & $3.8 \%$ & $75.0 \%$ & $11.1 \%$ & $68.8 \%$ & $76.5 \%$ \\
\hline 25 & $57.5 \%$ & $63.8 \%$ & $51.3 \%$ & $57.6 \%$ & $10.0 \%$ & $11.3 \%$ & $38.1 \%$ & $27.3 \%$ & $61.1 \%$ & $37.5 \%$ \\
\hline 26 & $71.3 \%$ & $61.3 \%$ & $58.8 \%$ & $60.1 \%$ & $8.8 \%$ & $3.8 \%$ & $50.0 \%$ & $10.0 \%$ & $68.4 \%$ & $58.8 \%$ \\
\hline 27 & $72.5 \%$ & $57.5 \%$ & $63.8 \%$ & $60.7 \%$ & $7.5 \%$ & $7.5 \%$ & $37.5 \%$ & $18.2 \%$ & $69.2 \%$ & $88.9 \%$ \\
\hline 28 & $75.0 \%$ & $58.8 \%$ & $62.5 \%$ & $60.7 \%$ & $10.0 \%$ & $5.0 \%$ & $42.1 \%$ & $11.1 \%$ & $90.9 \%$ & $71.4 \%$ \\
\hline 29 & $72.5 \%$ & $60.0 \%$ & $68.8 \%$ & $64.4 \%$ & $6.3 \%$ & $6.3 \%$ & $55.6 \%$ & $15.6 \%$ & $62.5 \%$ & $69.6 \%$ \\
\hline 30 & $65.0 \%$ & $66.3 \%$ & $62.5 \%$ & $64.4 \%$ & $3.8 \%$ & $7.5 \%$ & $33.3 \%$ & $18.8 \%$ & $66.7 \%$ & $50.0 \%$ \\
\hline 31 & $80.0 \%$ & $62.5 \%$ & $68.8 \%$ & $65.7 \%$ & $8.8 \%$ & $3.8 \%$ & $53.8 \%$ & $7.9 \%$ & $75.0 \%$ & $76.5 \%$ \\
\hline 32 & $77.5 \%$ & $63.8 \%$ & $67.5 \%$ & $65.7 \%$ & $8.8 \%$ & $5.0 \%$ & $46.7 \%$ & $10.0 \%$ & $81.8 \%$ & $71.4 \%$ \\
\hline 33 & $70.0 \%$ & $73.8 \%$ & $61.3 \%$ & $67.6 \%$ & $2.5 \%$ & $10.0 \%$ & $25.0 \%$ & $22.2 \%$ & $69.6 \%$ & $76.9 \%$ \\
\hline 34 & $77.5 \%$ & $71.3 \%$ & $70.0 \%$ & $70.7 \%$ & $10.0 \%$ & $5.0 \%$ & $61.5 \%$ & $8.7 \%$ & $54.5 \%$ & $60.0 \%$ \\
\hline All & $58.7 \%$ & $45.3 \%$ & $45.9 \%$ & $45.6 \%$ & $13.0 \%$ & $4.9 \%$ & $38.2 \%$ & $19.3 \%$ & $63.4 \%$ & $61.2 \%$ \\
\hline
\end{tabular}

\section{EC.3.1. Choice percentages}

Both the null and mixed error models require that $P(H)$ as a function of $\frac{p_{H H}+p_{L L}}{2}$ be symmetric around $p_{H H}=p_{L L}$, and hence symmetric around $\frac{\% H^{+}+\% H^{-}}{2}$. Let $\% H, \% H^{+}$, and $\% H^{-}$denote the percentage of participants choosing $H$ over $L, H^{+}$over $L^{+}$and $H^{-}$over $L^{-}$, respectively. The discussion above indicates that symmetry around $p_{H H}=p_{L L}$ is identical to symmetry around $\frac{\% H^{+}+\% H^{-}}{2}=\frac{1}{2}$. Figure EC.4A plots $\% H$ against $\frac{\% H^{+}+\% H^{-}}{2}$. Figure EC.4A shows a clear asymmetry around $\frac{\% H^{+}+\% H^{-}}{2}=\frac{1}{2}$. To test for this asymmetry statistically, we regress $\% H-.5$ against $\frac{\% H^{+}+\% H^{-}}{2}-.5$, using each of the 34 double matching tests as an observation. The regression line is also shown in Figure EC.4A. The null and mixed error models predict that the intercept should be 0 , whereas the asymmetric model predicts a positive intercept. We find a positive intercept $\left(\beta_{0}=.124, t(32)=8.95\right)$, consistent with the asymmetric model.

\section{EC.3.2. Double Matching Violation Rates}

The null and mixed error models imply that $P\left(H L^{+} L^{-}\right)=P\left(L H^{+} H^{-}\right)$for $p_{H H}=p_{L L}$. In contrast, the asymmetric model implies that $P\left(H L^{+} L^{-}\right)>P\left(L H^{+} H^{-}\right)$for $p_{H H}=p_{L L}$ if $\epsilon_{H}>\epsilon_{S}=$ 
Figure EC.4 Empirical data on choice percentages, double matching violation rates, error rates, and conditional probabilities.
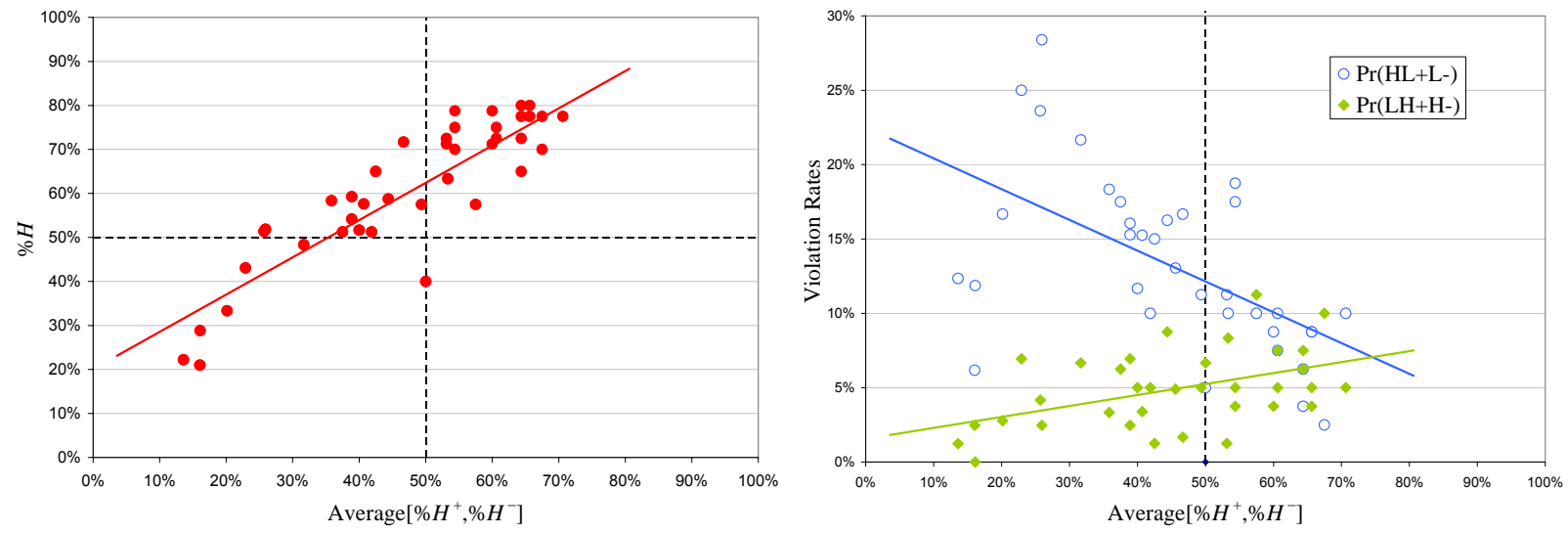

A: Choice Percentages
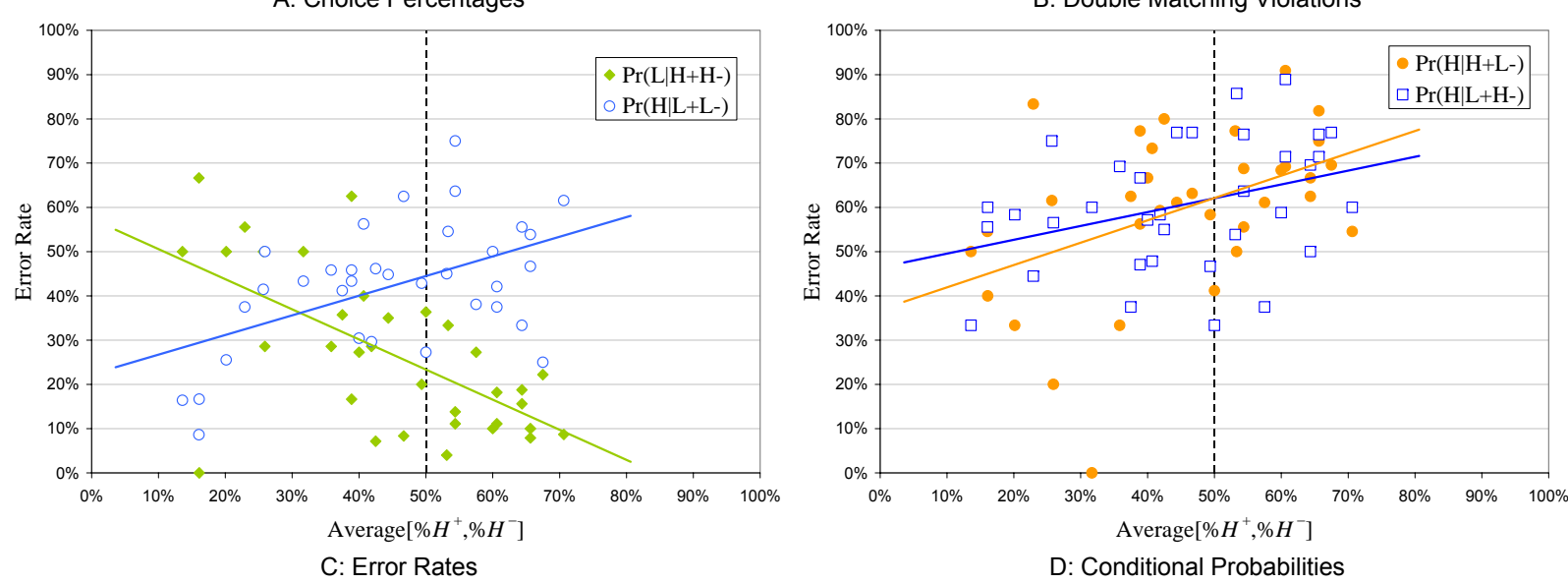

Note. Panel A illustrates choice percentages, $\% H$, as a function of the average of $\% H^{+}$and $\% H^{-}$. The empirical data show an asymmetry in that $\% H>\frac{\% H^{+}+\% H^{-}}{2}$ for $\frac{\% H^{+}+\% H^{-}}{2}=\frac{1}{2}$. Panel B depicts double matching violation rates, $\% H L^{+} L^{-}$and $\% L H^{+} H^{-}$, as a function of the average of $\% H^{+}$and $\% H^{-}$. The empirical data show an asymmetry in that $\% H L^{+} L^{-}>\% L H^{+} H^{-}$for $\frac{\% H^{+}+\% H^{-}}{2}=\frac{1}{2}$. Panel $\mathrm{C}$ shows error rates, $P\left(H \mid L^{+} L^{-}\right)$and $P\left(L \mid H^{+} H^{-}\right)$, as a function of the average of $\% H^{+}$and $\% H^{-}$. The empirical data show an asymmetry in that $P\left(H \mid L^{+} L^{-}\right)>P\left(L \mid H^{+} H^{-}\right)$ for $\frac{\% H^{+}+\% H^{-}}{2}=\frac{1}{2}$. Panel D illustrates conditional probabilities, $P\left(H \mid H^{+} L^{-}\right)$and $P\left(H \mid L^{+} H^{-}\right)$as a function of the average of $\% H^{+}$and $\% H^{-}$. The empirical data show an asymmetry in that $P\left(H \mid H^{+} L^{-}\right)>\frac{1}{2}$ and $P\left(H \mid L^{+} H^{-}\right)>\frac{1}{2}$ for $\frac{\% H^{+}+\% H^{-}}{2}=\frac{1}{2}$.

$\epsilon_{L}$. Let $\% H L^{+} L^{-}$and $\% L H^{+} H^{-}$denote the percentage of double matching violations, $H L^{+} L^{-}$ and $\mathrm{LH}^{+} \mathrm{H}^{-}$, respectively. Figure EC.4B plots the double matching violation rates as a function of $\frac{\% H^{+}+\% H^{-}}{2}$ and indicates an asymmetry in double matching violations consistent with the asymmetric model (regression lines are added to help visualize the asymmetry). We test for this asymmetry statistically by regressing the difference between the double matching violation rates, $\% H L^{+} L^{-}-\% L H^{+} H^{-}$, against $\frac{\% H^{+}+\% H^{-}}{2}-.5$. Both the null and mixed error models predict that the intercept should be 0 , whereas the asymmetric model predicts a positive intercept. The regression produces a positive intercept consistent with the asymmetric model $\left(\beta_{0}=.069, t(32)=6.78\right)$.

EC.3.3. Error rates: $P\left(H \mid L^{+} L^{-}\right)$and $P\left(L \mid H^{+} H^{-}\right)$

Both the null and mixed error models require that $P\left(H \mid L^{+} L^{-}\right)=P\left(L \mid H^{+} H^{-}\right)$if $p_{H H}=p_{L L}$, whereas the asymmetric model requires that $P\left(H \mid L^{+} L^{-}\right)>P\left(L \mid H^{+} H^{-}\right)$if $p_{H H}=p_{L L}$. Figure EC.4C plots the two error rates as a function of $\frac{\% H^{+}+\% H^{-}}{2}$. We see an asymmetry consistent with 
the asymmetric model. We regress the difference $P\left(H \mid L^{+} L^{-}\right)-P\left(L \mid H^{+} H^{-}\right)$against $\frac{\% H^{+}+\% H^{-}}{2}$. The null error model and the mixed model predict a zero intercept. In contrast, the asymmetric model predicts a positive intercept. We find a positive intercept, consistent with the asymmetric model $\left(\beta_{0}=.211, t(32)=5.78\right)$.

\section{EC.3.4. Likelihood of choosing $H$ for "indeterminate" patterns}

The null and error models again predict a symmetry around $p_{H H}=p_{L L}: P\left(H \mid H^{+} L^{-}\right)=$ $P\left(H \mid L^{+} H^{-}\right)=.5$. On the other hand, the asymmetric model predicts that $P\left(H \mid H^{+} L^{-}\right)=$ $P\left(H \mid L^{+} H^{-}\right)>.5$ for $p_{H H}=p_{L L}$. Figure EC.4D plots both error rates as a function of $\frac{\% H^{+}+\% H^{-}}{2}$. The data reveal an asymmetry consistent with the asymmetric model. A regression of $P\left(H \mid H^{+} L^{-}\right)-.5$ and $P\left(H \mid L^{+} H^{-}\right)-.5$ against $\frac{\% H^{+}+\% H^{-}}{2}-.5$ produces a positive intercept consistent with the asymmetric model $\left(P\left(H \mid H^{+} L^{-}\right): \beta_{0}=.121, t(32)=3.98 ; P\left(H \mid L^{+} H^{-}\right): \beta_{0}=.120\right.$, $t(32)=4.85)$.

\section{EC.4. Likelihood ratio test for Analysis of Study 1 Data \\ EC.4.1. Procedure}

To test the significance of each double matching violation, we employ a likelihood ratio test. We first describe the details of the procedure and then provide the results of the statistical test.

The full set of data $D$ to be fit is found in Table EC.4. Let $\mathbf{p}$ denote the vector of probabilities of the underlying types. Furthermore, let $L_{0}(D ; \mathbf{p}, \epsilon)$ be the likelihood of the data under the null model for a given set of type parameters $\mathbf{p}$ and error rate, $\epsilon=\epsilon_{S}=\epsilon_{H}=\epsilon_{L}$, with $L_{0}^{*}(D)=\max _{\mathbf{p}, \epsilon} L_{0}(D \mid \mathbf{p}, \epsilon)$ the maximum likelihood for the null model. We also perform a maximum likelihood procedure on the mixed error model and the asymmetric error model and compare whether the extra parameter used in these models improves the fit of the model using a likelihood ratio test. For example, let $L_{A}\left(D ; \mathbf{p}, \epsilon_{\bar{H}}, \epsilon_{H}\right)$ be the likelihood of the data under the asymmetric model, with $L_{A}^{*}(D)=$ $\max _{\mathbf{p}, \epsilon_{\bar{H}}, \epsilon_{H}} L_{A}\left(D \mid \mathbf{p}, \epsilon_{\bar{H}}, \epsilon_{H}\right)$ the maximum likelihood for the asymmetric model (where $0 \leq \epsilon_{\bar{H}} \leq$ $\left.\epsilon_{H} \leq \frac{1}{2}\right)$. Then the statistic $2 \ln \left[L_{A}^{*}(D) / L_{0}^{*}(D)\right]$ is distributed approximately $\chi^{2}(1)$ (e.g., Mood and Graybill, 1963). The general error model has three error rate parameters, and thus a comparison of the general model and the null model uses $\chi^{2}(2)$.

\section{EC.4.2. Results}

We fit the choice patterns for each of the 34 double matching tests using the maximum likelihood procedure outlined in the previous section. We fit the mixed model under two different restrictions, $\epsilon_{M} \geq \epsilon_{S}$ and $\epsilon_{M} \leq \epsilon_{S}$. Table EC.5 contains a comparison of all models relative to the null error model. The table also compares the general model with the asymmetric model and the mixed model.

The asymmetric model fits the choice patterns better than either the mixed model or the general model (adjusting for the extra degree of freedom), showing a significantly better fit than the null model in $71 \%$ of the tests (using the conventional $p<.05$ standard). In contrast, the mixed model provides a significantly better fit than the null model in only $41 \%$ of the tests when $\epsilon_{S} \leq \epsilon_{M}$ and in $15 \%$ of the tests when $\epsilon_{S} \geq \epsilon_{M}$. In addition, the general model improves over the asymmetric model in only 1 of the 34 tests.

It is also instructive to look at the estimated error rates. Table EC.6 lists the estimated error rates for the null, asymmetric, mixed (with $\epsilon_{S} \leq \epsilon_{M}$ ), and general models. The asymmetric model yields qualitatively consistent error rates: in 33 of the 34 tests, $\epsilon_{H}>\epsilon_{\bar{H}}$. In contrast, the error rates for the mixed error model often encounter the constraint: in 17 of the 34 tests, $\epsilon_{M}=\epsilon_{S}$. Moreover, this pattern appears systematic. For the double matching tests in which $\frac{\% H^{+}+\% H^{-}}{2}<\frac{1}{2}, 16$ of 19 produce $\epsilon_{M}>\epsilon_{S}$, whereas $\epsilon_{M}=\epsilon_{S}$ in only 1 of the 14 tests in which $\frac{\% H^{+}+\% H^{-}}{2}>\frac{1}{2}$. Finally, the 
Table EC.4 Choice percentages for the 8 possible choice patterns for 34 tests of double matching (Study 1)

\begin{tabular}{|c|c|c|c|c|c|c|c|c|c|}
\hline & & \multicolumn{8}{|c|}{ Choice Patterns } \\
\hline Test & $\frac{\% H^{+}+\% H^{-}}{2}$ & $\% H H^{+} H^{-}$ & $\% H H^{+} L^{-}$ & $\% H L^{+} H^{-}$ & $\% H L^{+} L^{-}$ & $\% L H^{+} H^{-}$ & $\% L H^{+} L^{-}$ & $\% L L^{+} H^{-}$ & $\% L L^{+} L^{-}$ \\
\hline 1 & $13.6 \%$ & $1.2 \%$ & $3.7 \%$ & $4.9 \%$ & $12.3 \%$ & $1.2 \%$ & $3.7 \%$ & $9.9 \%$ & $63.0 \%$ \\
\hline 2 & $16.0 \%$ & $1.2 \%$ & $7.4 \%$ & $6.2 \%$ & $6.2 \%$ & $2.5 \%$ & $6.2 \%$ & $4.9 \%$ & $65.4 \%$ \\
\hline 3 & $16.1 \%$ & $3.4 \%$ & $3.4 \%$ & $10.2 \%$ & $11.9 \%$ & $0.0 \%$ & $5.1 \%$ & $6.8 \%$ & $59.3 \%$ \\
\hline 4 & $20.1 \%$ & $2.8 \%$ & $4.2 \%$ & $9.7 \%$ & $16.7 \%$ & $2.8 \%$ & $8.3 \%$ & $6.9 \%$ & $48.6 \%$ \\
\hline 5 & $22.9 \%$ & $5.6 \%$ & $6.9 \%$ & $5.6 \%$ & $25.0 \%$ & $6.9 \%$ & $1.4 \%$ & $6.9 \%$ & $41.7 \%$ \\
\hline 6 & $25.7 \%$ & $4.2 \%$ & $11.1 \%$ & $12.5 \%$ & $23.6 \%$ & $4.2 \%$ & $6.9 \%$ & $4.2 \%$ & $33.3 \%$ \\
\hline 7 & $25.9 \%$ & $6.2 \%$ & $1.2 \%$ & $16.0 \%$ & $28.4 \%$ & $2.5 \%$ & $4.9 \%$ & $12.3 \%$ & $28.4 \%$ \\
\hline 8 & $31.7 \%$ & $6.7 \%$ & $0.0 \%$ & $20.0 \%$ & $21.7 \%$ & $6.7 \%$ & $3.3 \%$ & $13.3 \%$ & $28.3 \%$ \\
\hline 9 & $35.8 \%$ & $8.3 \%$ & $1.7 \%$ & $30.0 \%$ & $18.3 \%$ & $3.3 \%$ & $3.3 \%$ & $13.3 \%$ & $21.7 \%$ \\
\hline 10 & $37.5 \%$ & $11.3 \%$ & $18.8 \%$ & $3.8 \%$ & $17.5 \%$ & $6.3 \%$ & $11.3 \%$ & $6.3 \%$ & $25.0 \%$ \\
\hline 12 & $38.9 \%$ & $4.2 \%$ & $12.5 \%$ & $9.9 \%$ & $15.3 \%$ & $6.9 \%$ & $9.7 \%$ & $11.1 \%$ & $18.1 \%$ \\
\hline 11 & $38.9 \%$ & $12.3 \%$ & $21.0 \%$ & $22.2 \%$ & $16.0 \%$ & $2.5 \%$ & $6.2 \%$ & $11.1 \%$ & $21.0 \%$ \\
\hline 13 & $40.0 \%$ & $13.3 \%$ & $13.3 \%$ & $13.3 \%$ & $11.7 \%$ & $5.0 \%$ & $6.7 \%$ & $10.0 \%$ & $26.7 \%$ \\
\hline 14 & $40.7 \%$ & $5.1 \%$ & $18.6 \%$ & $18.6 \%$ & $15.3 \%$ & $3.4 \%$ & $6.8 \%$ & $20.3 \%$ & $11.9 \%$ \\
\hline 15 & $41.9 \%$ & $12.5 \%$ & $20.0 \%$ & $8.8 \%$ & $10.0 \%$ & $5.0 \%$ & $13.8 \%$ & $6.3 \%$ & $23.8 \%$ \\
\hline 16 & $42.5 \%$ & $16.3 \%$ & $20.0 \%$ & $13.8 \%$ & $15.0 \%$ & $1.3 \%$ & $5.0 \%$ & $11.3 \%$ & $17.5 \%$ \\
\hline 17 & $44.4 \%$ & $16.3 \%$ & $13.8 \%$ & $12.5 \%$ & $16.3 \%$ & $8.8 \%$ & $8.8 \%$ & $3.8 \%$ & $20.0 \%$ \\
\hline 18 & $46.7 \%$ & $18.3 \%$ & $20.0 \%$ & $16.7 \%$ & $16.7 \%$ & $1.7 \%$ & $11.7 \%$ & $5.0 \%$ & $10.0 \%$ \\
\hline 19 & $49.4 \%$ & $20.0 \%$ & $17.5 \%$ & $8.8 \%$ & $11.3 \%$ & $5.0 \%$ & $12.5 \%$ & $10.0 \%$ & $15.0 \%$ \\
\hline 20 & $50.0 \%$ & $11.7 \%$ & $11.7 \%$ & $11.7 \%$ & $5.0 \%$ & $6.7 \%$ & $16.7 \%$ & $23.3 \%$ & $13.3 \%$ \\
\hline 21 & $53.1 \%$ & $30.0 \%$ & $21.3 \%$ & $8.8 \%$ & $11.3 \%$ & $1.3 \%$ & $6.3 \%$ & $7.5 \%$ & $13.8 \%$ \\
\hline 22 & $53.3 \%$ & $16.7 \%$ & $16.7 \%$ & $20.0 \%$ & $10.0 \%$ & $8.3 \%$ & $16.7 \%$ & $3.3 \%$ & $8.3 \%$ \\
\hline 23 & $54.4 \%$ & $31.3 \%$ & $12.5 \%$ & $8.8 \%$ & $17.5 \%$ & $5.0 \%$ & $10.0 \%$ & $5.0 \%$ & $10.0 \%$ \\
\hline 24 & $54.4 \%$ & $30.0 \%$ & $13.8 \%$ & $16.3 \%$ & $18.8 \%$ & $3.8 \%$ & $6.3 \%$ & $5.0 \%$ & $6.3 \%$ \\
\hline 25 & $57.5 \%$ & $30.0 \%$ & $13.8 \%$ & $3.8 \%$ & $10.0 \%$ & $11.3 \%$ & $8.8 \%$ & $6.3 \%$ & $16.3 \%$ \\
\hline 26 & $60.0 \%$ & $33.8 \%$ & $16.3 \%$ & $12.5 \%$ & $8.8 \%$ & $3.8 \%$ & $7.5 \%$ & $8.8 \%$ & $8.8 \%$ \\
\hline 27 & $60.6 \%$ & $33.8 \%$ & $11.3 \%$ & $20.0 \%$ & $7.5 \%$ & $7.5 \%$ & $5.0 \%$ & $2.5 \%$ & $12.5 \%$ \\
\hline 28 & $60.6 \%$ & $40.0 \%$ & $12.5 \%$ & $12.5 \%$ & $10.0 \%$ & $5.0 \%$ & $1.3 \%$ & $5.0 \%$ & $13.8 \%$ \\
\hline 29 & $64.4 \%$ & $33.8 \%$ & $12.5 \%$ & $20.0 \%$ & $6.3 \%$ & $6.3 \%$ & $7.5 \%$ & $8.8 \%$ & $5.0 \%$ \\
\hline 30 & $64.4 \%$ & $32.5 \%$ & $17.5 \%$ & $11.3 \%$ & $3.8 \%$ & $7.5 \%$ & $8.8 \%$ & $11.3 \%$ & $7.5 \%$ \\
\hline 31 & $65.6 \%$ & $43.8 \%$ & $11.3 \%$ & $16.3 \%$ & $8.8 \%$ & $3.8 \%$ & $3.8 \%$ & $5.0 \%$ & $7.5 \%$ \\
\hline 32 & $65.6 \%$ & $45.0 \%$ & $11.3 \%$ & $12.5 \%$ & $8.8 \%$ & $5.0 \%$ & $2.5 \%$ & $5.0 \%$ & $10.0 \%$ \\
\hline 33 & $67.5 \%$ & $35.0 \%$ & $20.0 \%$ & $12.5 \%$ & $2.5 \%$ & $10.0 \%$ & $8.8 \%$ & $3.8 \%$ & $7.5 \%$ \\
\hline 34 & $70.6 \%$ & $52.5 \%$ & $7.5 \%$ & $7.5 \%$ & $10.0 \%$ & $5.0 \%$ & $6.3 \%$ & $5.0 \%$ & $6.3 \%$ \\
\hline
\end{tabular}

general model appears to be too flexible: in 22 of 34 tests, one of the three parameters encounters at least one of the constraints.

As a further test of whether each error model is "too flexible," we fit the choice patterns for the aggregate data (all 34 double matching tests) with a single set of error rates for each error model, while allowing the probability of individual types to differ for each test (see "All" row in Table EC.6). Thus, the null error model has 103 parameters (34 tests times 3 type parameters for each test +1 error rate parameter), whereas the mixed and asymmetric models have 104 parameters, and the general model has 105 parameters. For the aggregate data, the mixed, asymmetric, and general model all improve on the null model. Interestingly, the general model is only marginally significantly better than the asymmetric model. Error rates for this test are also found in Table EC.6. We next use the estimates from this analysis to examine the four implications discussed in the previous section. We compare the null error model, the mixed model, and the asymmetric model. Consider, for example, the implications for choice percentages, $\% H$ and $\frac{\% H^{+}+\% H^{-}}{2}$. Figure EC.5 plots the actual choice percentages, as well as a regression line to summarize the relationship (this 
Table EC.5 Error model comparisons using likelihood ratio analysis (Study 1).

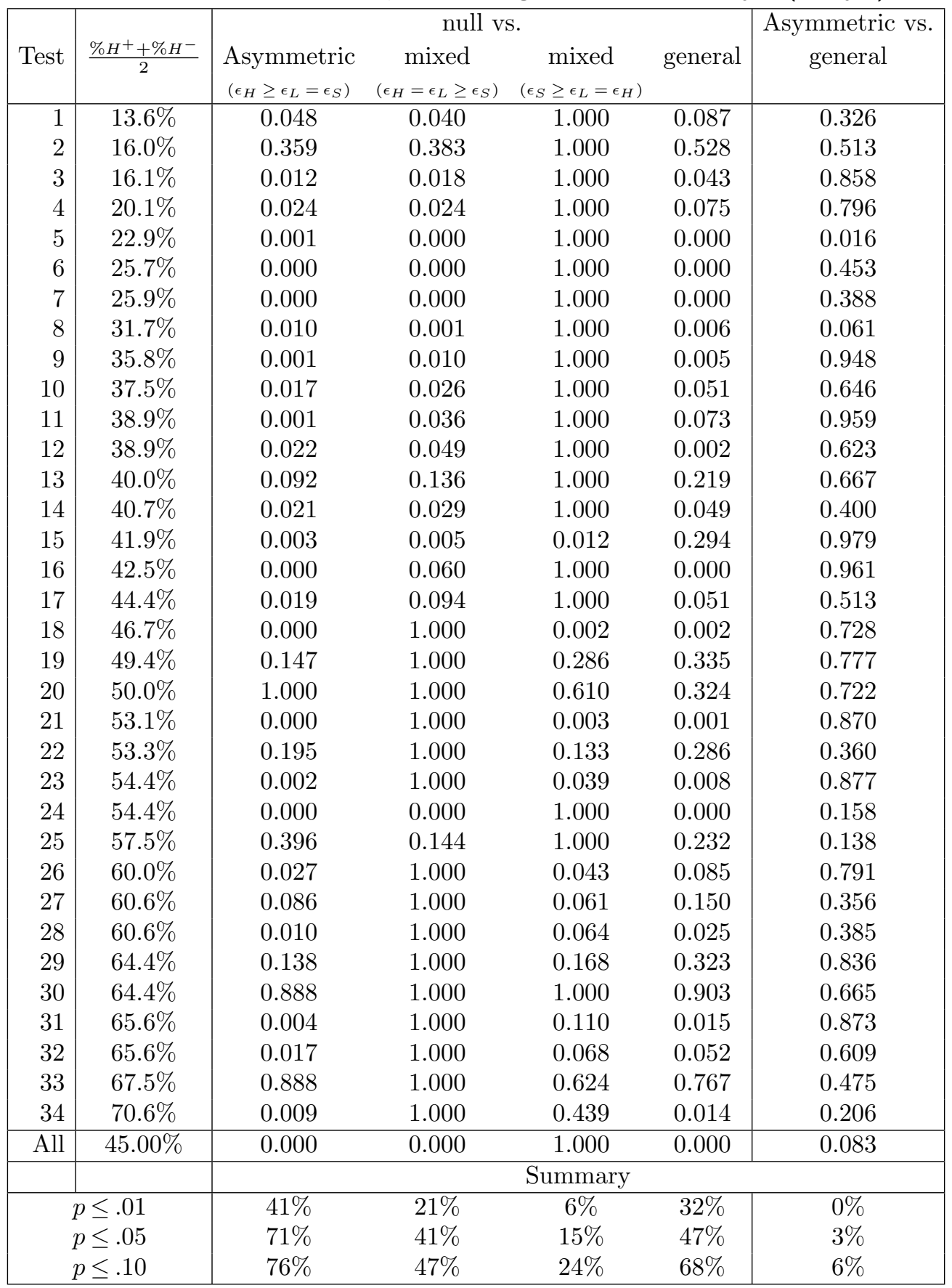

The last five columns shows $p$-values for comparisons of nested models. The summary at the bottom of the table indicates what percentage of the 34 tests are significant at various $p$ levels.

part of the figure is identical to Figure EC.4A). For each error model, we use the parameter estimates to calculate, $P(H)$ and $\frac{P\left(H^{+}\right)+P\left(H^{-}\right)}{2}$. We then regress the estimated values of $\frac{P\left(H^{+}\right)+P\left(H^{-}\right)}{2}$ on the estimated values of $P(H)$. Figure EC.5 also shows the regression lines for the null, mixed, and asymmetric models. The asymmetric model shows a close correspondence to the actual data, whereas the null error model and the mixed error model largely underpredict $\% H$. We conduct a runs test to test this underprediction formally. For each model, an underprediction is coded as 
Figure EC.5 Choice Percentages, Comparison of empirical data with error models (Study 1).

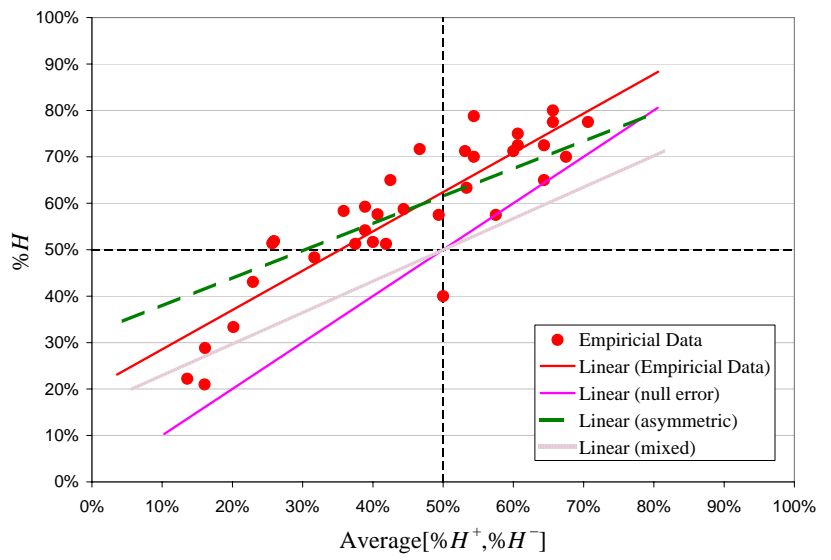

" -1 " and an overprediction is coded as " +1 ". We then tally the number of alternations (from "-1" to " +1 " or from " +1 " to " $-1 ")$. The runs test rejects the null and mixed error models, but not the asymmetric model $(p<.05)$.

We perform a similar analysis on the double matching violations. Figure EC.6 depicts the empirical data compared with estimates from the null error model, the mixed error model, and the asymmetric model. The empirical data reveal a distinct asymmetry captured only by the asymmetric model: the violation rate $\% H L^{+} L^{-}$exceeds the violation rate $\% L^{+} H^{-}$for double matching tests in which $\frac{\% H^{+}+\% H^{-}}{2}>\frac{1}{2}$. A runs test rejects the mixed model for both types of double matching violations and the null error model for $\mathrm{HL}^{+} L^{-}$violations $(p<.05)$. The asymmetric model is not rejected for either type of violation.

We next consider the error rates, $P\left(H \mid L^{+} L^{-}\right)$and $P\left(L \mid H^{+} H^{-}\right)$. Figure EC.7 depicts the empirical data relative to the estimates from the null, mixed, and asymmetric models. Again, the empirical data reveal an asymmetry: $P\left(H \mid L^{+} L^{-}\right)>P\left(L \mid H^{+} H^{-}\right)$for $\frac{\% H^{+}+\% H^{-}}{2}<\frac{1}{2}$, a pattern predicted only by the asymmetric model. A runs tests rejects the null error model for both error rates and the mixed error model for the $P\left(H \mid L^{+} L^{-}\right)$error rate $(p<.05)$. The asymmetric model is not rejected for either error rate.

Finally, we turn to the probability of choosing $H$ for the indeterminate patterns, $H^{+} L^{-}$and $L^{+} H^{-}$. Figure EC.8 contains the empirical data for these measures, as well as the estimates from the various error models. Again, the asymmetric model shows a close correspondence to the empirical data, whereas the null error model and the mixed error model largely underestimate the conditional probabilities. Once again, a runs test rejects both the null and mixed error models, but not the asymmetric model $(p<.05)$.

\section{EC.5. Robustness Analysis for Weighting Function Estimation: Study 1}

In Section 3.4 of $\mathrm{Wu}$ and Markle, we formally tested the hypothesis that violations of double matching result from a diminished sensitivity to probability differences for mixed gambles relative to gain or loss gambles by fitting a probability weighting function to our double matching test data. This section presents robustness analyses.

The estimation results support our hypothesis of greater curvature for mixed gambles. Our weighting function estimate for single-domain gambles, $\hat{\gamma}_{S}$, was .67, close to the parameter estimate of .71 from $\mathrm{Wu}$ and Gonzalez (1996). In contrast, the parameter value for mixed gambles was considerably lower, $\hat{\gamma}_{M}=.55$, a difference that was statistically significant $(t=620.9, p<.0001)$. (The best-fitting scaling parameter was found to be $\hat{\mu}=.18$.) We conducted a variety of sensitivity 
Figure EC.6 Double Matching Violations, Comparison of empirical data with error models (Study 1).

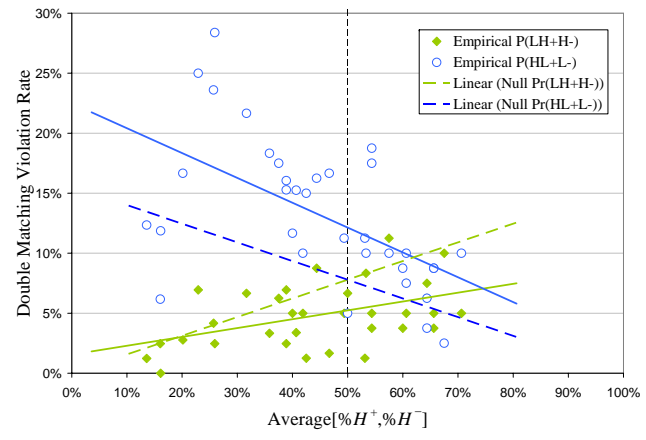

A: Double Matching Violations: Null

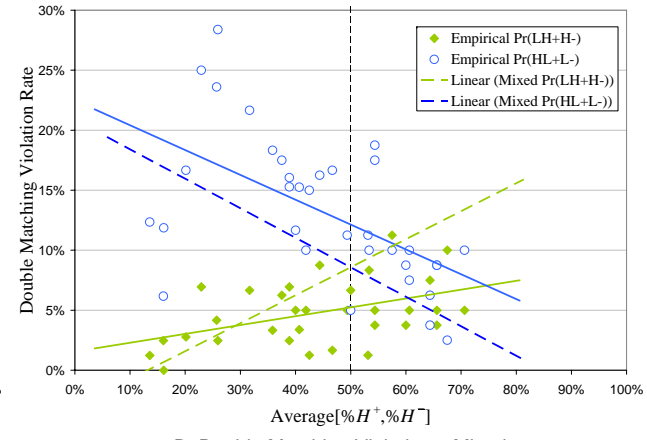

B: Double Matching Violations: Mixed

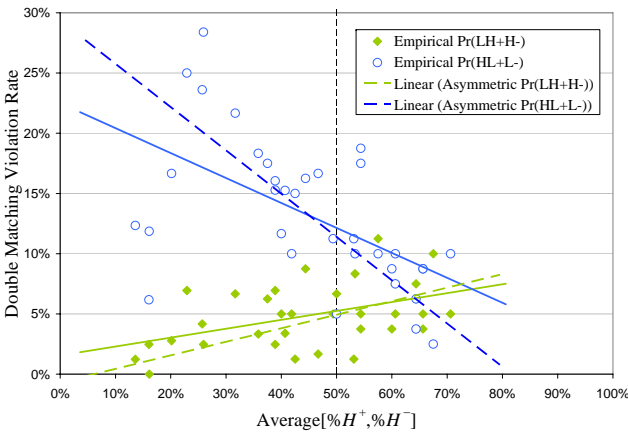

C: Double Matching Violations: Asymmetric

Note. Panel A: null error model; Panel B: mixed error model; Panel C: asymmetric error model

Figure EC.7 Error Rates, Comparison of empirical data with error models. (Study 1)
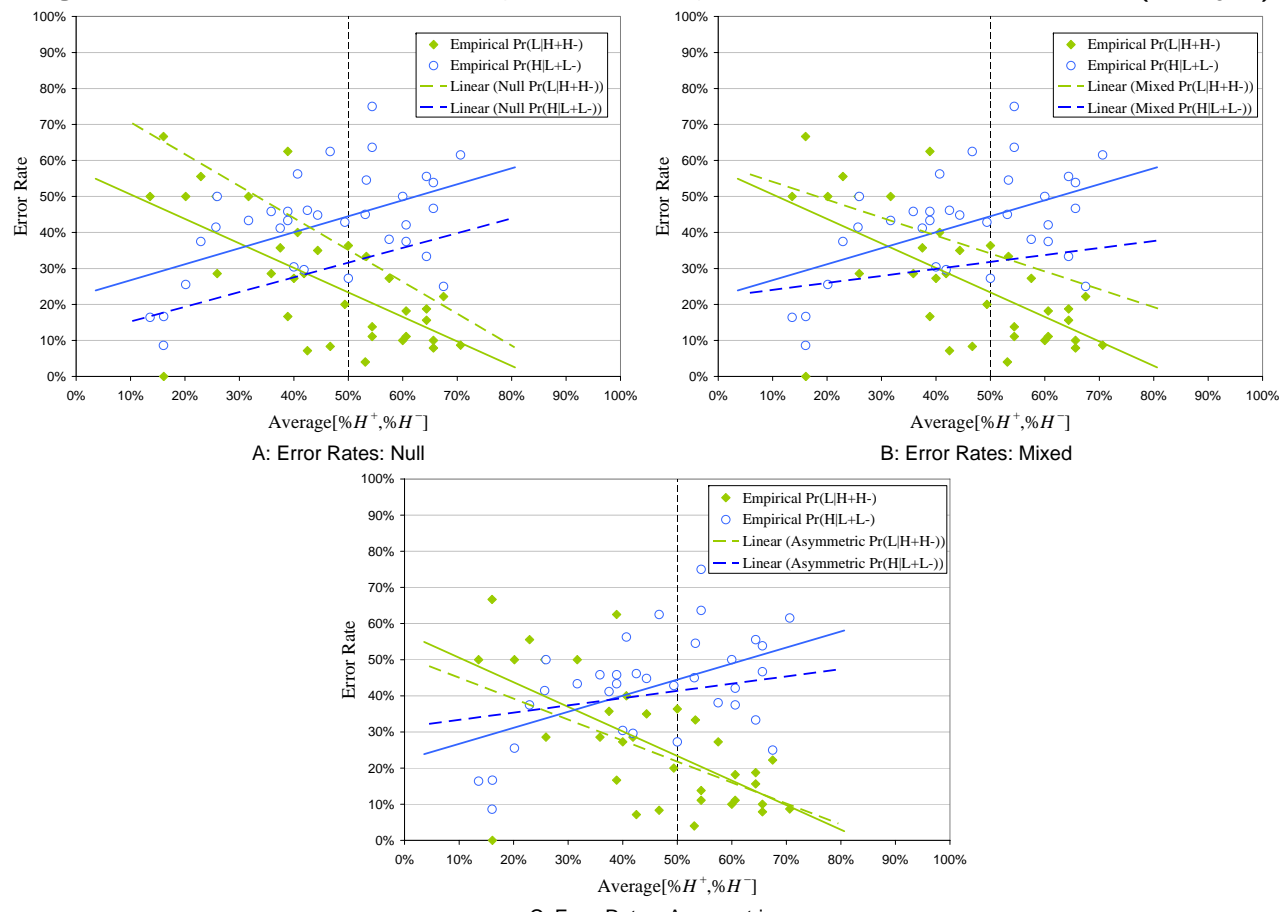

C: Error Rates: Asymmetric

Note. Error rates shown are: $P\left(H \mid L^{+} L^{-}\right)$and $P\left(L \mid H^{+} H^{-}\right)$. Panel A: null error model; Panel B: mixed error model; Panel C: asymmetric error model 
Table EC.6 Estimated error rates using likelihood ratio analysis for various error models (Study 1)

\begin{tabular}{|c|c|c|c|c|c|c|c|c|c|c|c|c|c|}
\hline & & \multicolumn{3}{|c|}{ Null } & \multicolumn{3}{|c|}{ Asymmetric } & \multicolumn{3}{|c|}{$\operatorname{Mixed}\left(\epsilon_{S} \leq \epsilon_{H}=\epsilon_{L}\right)$} & \multicolumn{3}{|c|}{ General } \\
\hline Gamble & $\frac{\% H^{+}+\% H^{-}}{2}$ & $\epsilon_{S}$ & $\epsilon_{L}$ & $\epsilon_{H}$ & $\epsilon_{S}$ & $\epsilon_{L}$ & $\epsilon_{H}$ & $\epsilon_{S}$ & $\epsilon_{L}$ & $\epsilon_{H}$ & $\epsilon_{S}$ & $\epsilon_{L}$ & $\epsilon_{H}$ \\
\hline 1 & $13.6 \%$ & 0.106 & 0.106 & 0.106 & 0.062 & 0.062 & 0.160 & 0.048 & 0.164 & 0.164 & 0.000 & 0.419 & 0.165 \\
\hline 2 & $16.0 \%$ & 0.063 & 0.063 & 0.063 & 0.048 & 0.048 & 0.088 & 0.043 & 0.088 & 0.088 & 0.000 & 0.275 & 0.095 \\
\hline 3 & $16.1 \%$ & 0.089 & 0.089 & 0.089 & 0.009 & 0.009 & 0.163 & 0.000 & 0.159 & 0.159 & 0.014 & 0.000 & 0.163 \\
\hline 4 & $20.1 \%$ & 0.173 & 0.173 & 0.173 & 0.111 & 0.111 & 0.257 & 0.075 & 0.259 & 0.259 & 0.127 & 0.000 & 0.255 \\
\hline 5 & $22.9 \%$ & 0.237 & 0.237 & 0.237 & 0.156 & 0.156 & 0.397 & 0.000 & 0.404 & 0.404 & 0.000 & 0.450 & 0.394 \\
\hline 6 & $25.7 \%$ & 0.286 & 0.286 & 0.286 & 0.129 & 0.129 & 0.437 & 0.000 & 0.426 & 0.426 & 0.000 & 0.304 & 0.448 \\
\hline 7 & $25.9 \%$ & 0.240 & 0.240 & 0.240 & 0.111 & 0.111 & 0.441 & 0.087 & 0.462 & 0.462 & 0.089 & 0.270 & 0.464 \\
\hline 8 & $31.7 \%$ & 0.215 & 0.215 & 0.215 & 0.128 & 0.128 & 0.369 & 0.050 & 0.431 & 0.431 & 0.050 & 0.404 & 0.436 \\
\hline 9 & $35.8 \%$ & 0.202 & 0.202 & 0.202 & 0.106 & 0.106 & 0.435 & 0.000 & 0.419 & 0.419 & 0.110 & 0.090 & 0.429 \\
\hline 10 & $37.5 \%$ & 0.236 & 0.236 & 0.236 & 0.177 & 0.177 & 0.381 & 0.000 & 0.396 & 0.396 & 0.156 & 0.249 & 0.400 \\
\hline 11 & $38.9 \%$ & 0.223 & 0.223 & 0.223 & 0.090 & 0.090 & 0.409 & 0.072 & 0.321 & 0.321 & 0.185 & 0.167 & 0.402 \\
\hline 12 & $38.9 \%$ & 0.239 & 0.239 & 0.239 & 0.184 & 0.184 & 0.409 & 0.055 & 0.500 & 0.500 & 0.163 & 0.000 & 0.385 \\
\hline 13 & $40.0 \%$ & 0.190 & 0.190 & 0.190 & 0.125 & 0.125 & 0.301 & 0.000 & 0.294 & 0.294 & 0.000 & 0.223 & 0.335 \\
\hline 14 & $40.7 \%$ & 0.194 & 0.194 & 0.194 & 0.140 & 0.140 & 0.417 & 0.121 & 0.500 & 0.500 & 0.122 & 0.319 & 0.500 \\
\hline 15 & $41.9 \%$ & 0.347 & 0.347 & 0.347 & 0.126 & 0.126 & 0.273 & 0.000 & 0.292 & 0.292 & 0.104 & 0.152 & 0.284 \\
\hline 16 & $42.5 \%$ & 0.200 & 0.200 & 0.200 & 0.044 & 0.044 & 0.433 & 0.000 & 0.325 & 0.325 & 0.000 & 0.076 & 0.451 \\
\hline 17 & $44.4 \%$ & 0.292 & 0.292 & 0.292 & 0.239 & 0.239 & 0.475 & 0.000 & 0.408 & 0.408 & 0.000 & 0.296 & 0.492 \\
\hline 18 & $46.7 \%$ & 0.266 & 0.266 & 0.266 & 0.067 & 0.067 & 0.500 & 0.266 & 0.266 & 0.266 & 0.132 & 0.025 & 0.500 \\
\hline 19 & $49.4 \%$ & 0.205 & 0.205 & 0.205 & 0.152 & 0.152 & 0.318 & 0.205 & 0.205 & 0.205 & 0.310 & 0.000 & 0.206 \\
\hline 20 & $50.0 \%$ & 0.137 & 0.137 & 0.137 & 0.137 & 0.137 & 0.138 & 0.138 & 0.138 & 0.138 & 0.149 & 0.206 & 0.000 \\
\hline 21 & $53.1 \%$ & 0.159 & 0.159 & 0.159 & 0.030 & 0.030 & 0.423 & 0.160 & 0.160 & 0.160 & 0.000 & 0.041 & 0.437 \\
\hline 22 & $53.3 \%$ & 0.271 & 0.271 & 0.271 & 0.227 & 0.227 & 0.450 & 0.271 & 0.271 & 0.271 & 0.384 & 0.095 & 0.000 \\
\hline 23 & $54.4 \%$ & 0.252 & 0.252 & 0.252 & 0.148 & 0.148 & 0.500 & 0.252 & 0.252 & 0.252 & 0.101 & 0.153 & 0.500 \\
\hline 24 & $54.4 \%$ & 0.500 & 0.500 & 0.500 & 0.100 & 0.100 & 0.500 & 0.322 & 0.322 & 0.322 & 0.334 & 0.066 & 0.500 \\
\hline 25 & $57.5 \%$ & 0.204 & 0.204 & 0.204 & 0.186 & 0.186 & 0.286 & 0.093 & 0.298 & 0.298 & 0.000 & 0.276 & 0.374 \\
\hline 26 & $60.0 \%$ & 0.159 & 0.159 & 0.159 & 0.083 & 0.083 & 0.391 & 0.159 & 0.159 & 0.159 & 0.000 & 0.108 & 0.452 \\
\hline 27 & $60.6 \%$ & 0.220 & 0.220 & 0.220 & 0.137 & 0.137 & 0.438 & 0.220 & 0.220 & 0.220 & 0.249 & 0.123 & 0.298 \\
\hline 28 & $60.6 \%$ & 0.180 & 0.180 & 0.180 & 0.095 & 0.095 & 0.454 & 0.180 & 0.180 & 0.180 & 0.173 & 0.083 & 0.400 \\
\hline 29 & $64.4 \%$ & 0.164 & 0.164 & 0.164 & 0.125 & 0.125 & 0.392 & 0.165 & 0.165 & 0.165 & 0.029 & 0.155 & 0.500 \\
\hline 30 & $64.4 \%$ & 0.132 & 0.132 & 0.132 & 0.129 & 0.129 & 0.152 & 0.132 & 0.133 & 0.133 & 0.000 & 0.184 & 0.348 \\
\hline 31 & $65.6 \%$ & 0.181 & 0.181 & 0.181 & 0.069 & 0.069 & 0.500 & 0.181 & 0.181 & 0.181 & 0.090 & 0.066 & 0.500 \\
\hline 32 & $65.6 \%$ & 0.175 & 0.175 & 0.175 & 0.090 & 0.090 & 0.459 & 0.175 & 0.175 & 0.175 & 0.171 & 0.088 & 0.391 \\
\hline 33 & $67.5 \%$ & 0.181 & 0.181 & 0.181 & 0.174 & 0.174 & 0.215 & 0.181 & 0.181 & 0.181 & 0.230 & 0.159 & 0.000 \\
\hline 34 & $70.6 \%$ & 0.151 & 0.151 & 0.151 & 0.079 & 0.079 & 0.441 & 0.152 & 0.152 & 0.152 & 0.000 & 0.099 & 0.494 \\
\hline All & $45.0 \%$ & 0.193 & 0.193 & 0.193 & 0.126 & 0.126 & 0.352 & 0.135 & 0.252 & 0.252 & 0.130 & 0.116 & 0.348 \\
\hline
\end{tabular}

Figure EC.8 Conditional Probabilities, Comparison of empirical data with error models (Study 1).
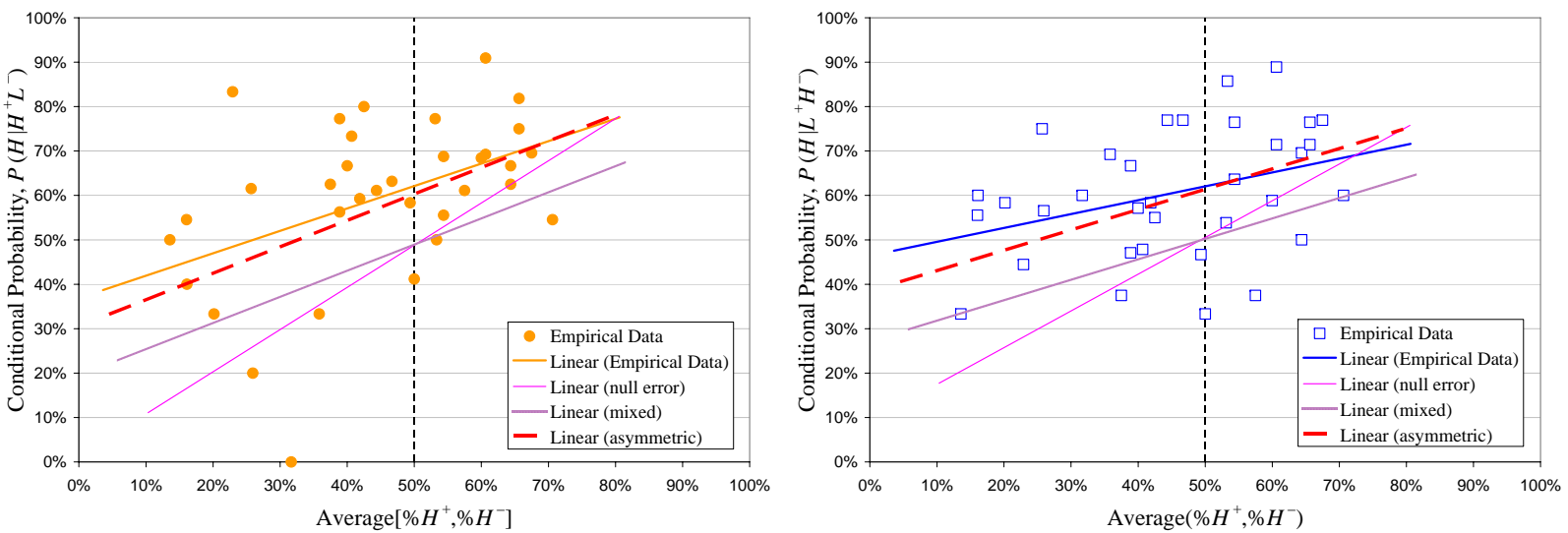

Note. Panel A depicts $P\left(H \mid H^{+} L^{-}\right)$, whereas Panel B illustrates $P\left(H \mid L^{+} H^{-}\right)$. 


Table EC.7
estimates (Tversky and Kahneman (1992) function) for
single-domain gambles and mixed gambles (Study 1).
\begin{tabular}{|c|ccccccc|c|}
\hline \multicolumn{7}{c}{ Estimates for single-domain gambles $\left(\gamma_{S}\right)$} \\
$\alpha \backslash \lambda$ & 1.00 & 1.25 & 1.50 & 1.75 & 2.00 & 2.25 & 2.50 \\
\hline 0.4 & 0.571 & 0.572 & 0.572 & 0.572 & 0.572 & 0.572 & 0.572 \\
0.5 & 0.684 & 0.681 & 0.678 & 0.676 & 0.675 & 0.673 & 0.672 \\
0.6 & 0.796 & 0.788 & 0.782 & 0.778 & 0.775 & 0.772 & 0.770 \\
0.7 & 0.910 & 0.896 & 0.886 & 0.879 & 0.874 & 0.870 & 0.867 \\
0.8 & 1.031 & 1.008 & 0.992 & 0.982 & 0.974 & 0.968 & 0.964 \\
0.9 & 1.162 & 1.126 & 1.103 & 1.087 & 1.076 & 1.068 & 1.062 \\
1.0 & 1.313 & 1.257 & 1.221 & 1.198 & 1.182 & 1.170 & 1.162 \\
\hline
\end{tabular}

\begin{tabular}{|c|ccccccc|}
\hline & \multicolumn{9}{|c|}{ Estimates for mixed gambles $\left(\gamma_{M}\right)$} \\
$\alpha \backslash \lambda$ & 1.00 & 1.25 & 1.50 & 1.75 & 2.00 & 2.25 & 2.50 \\
\hline 0.4 & 0.415 & 0.423 & 0.429 & 0.434 & 0.438 & 0.441 & 0.444 \\
0.5 & 0.530 & 0.536 & 0.541 & 0.544 & 0.547 & 0.549 & 0.550 \\
0.6 & 0.636 & 0.639 & 0.641 & 0.642 & 0.643 & 0.643 & 0.643 \\
0.7 & 0.734 & 0.734 & 0.734 & 0.733 & 0.731 & 0.730 & 0.728 \\
0.8 & 0.828 & 0.825 & 0.822 & 0.819 & 0.816 & 0.813 & 0.810 \\
0.9 & 0.917 & 0.912 & 0.908 & 0.903 & 0.899 & 0.895 & 0.891 \\
1.0 & 1.003 & 0.997 & 0.991 & 0.985 & 0.980 & 0.976 & 0.971 \\
\hline
\end{tabular}

Table shows estimates for combinations of $\alpha$ and $\lambda$. In all cases, differences between $\gamma_{S}$ and $\gamma_{M}$ are significant $(t \mathrm{~s}>100)$.

analyses to test whether this finding was robust. We found that $\hat{\gamma}_{M}$ was significantly lower than $\hat{\gamma}_{S}$ for all combinations of values of $\alpha$ from .3 to 1.0 and $\lambda$ from 1 to 2.5 (see Table EC.5).

To further investigate the robustness of our analysis, we replaced the Tversky and Kahneman (1992) function with the weighting function proposed by Prelec (1998), $\pi(p)=\exp \left(-(-\ln p)^{\beta}\right)$. The Prelec weighting function is the identity function when $\beta=1$, approaches a step function as $\beta \rightarrow 0$, and has a fixed point at $1 / e \approx .368$ (i.e., $\pi(1 / e)=1 / e$ ). We found qualitatively identical results $\left(\hat{\beta}_{S}=.61, \hat{\beta}_{M}=.47, t=589.6, p<.0001\right)$, with the Prelec function performing slightly worse in terms of log likelihood.

We also estimated a separate set of models in which we allowed $\gamma$ in the Tversky and Kahneman weighting function to differ for gain gambles, loss gambles, and mixed gambles. Specifically, we let $\gamma_{G S}, \gamma_{L S}, \gamma_{G M}=\gamma_{G S}-\delta$, and $\gamma_{L M}=\gamma_{L S}-\delta$, respectively, be the parameters for gain gambles, loss gambles, the gain portions of mixed gambles, and the loss portions of mixed gambles. Thus, $\delta$ captures the difference in curvature between mixed gambles and single-domain gambles. This specification yielded similar results to the analyses presented earlier. Contrary to most previous studies, the parameter for losses, $\hat{\gamma}_{L S}=.66$, was substantially lower than for gains, $\hat{\gamma}_{G S}=.76$ (however, see Baltussen et al., 2006), but most critically, the parameter capturing the difference between single-domain gambles and mixed gambles, $\hat{\delta}=.14$ was significantly positive $(t=587.9$, $p<.0001$ ), indicating a more curved weighting function for mixed gambles than for single-domain gambles.

\section{EC.6. Stochastic Choice Analysis of Study 2}

To test whether gain-loss separability is violated in Study 2, we develop two stochastic choice models. One model constrains preferences to follow gain-loss separability and one does not. We then test whether the unconstrained model provides a signficantly better fit to the choice data. We begin by making simplifying but standard assumption that the probability that prospect $S$ is chosen over prospect $T$ is captured by the logit model, $P(S \succ T)=\frac{1}{1+\exp (-\mu(U(S)-U(T)))}$. 
We fit the choice data using maximum likelihood. Thus, the likelihood function is:

$$
\begin{aligned}
& \prod P\left(H^{+} \succ A^{+}\right)^{(n)\left(\%\left(H^{+} \succ A^{+}\right)\right)} P\left(A^{+} \succ H^{+}\right)^{(n)\left(\%\left(A^{+} \succ H^{+}\right)\right)} \times \\
& \prod P\left(L^{+} \succ A^{+}\right)^{(n)\left(\%\left(L^{+} \succ A^{+}\right)\right)} P\left(A^{+} \succ L^{+}\right)^{(n)\left(\%\left(A^{+} \succ L^{+}\right)\right)} \times \\
& \prod P\left(\left[H^{+}, C^{-}\right] \succ B\right)^{(n)\left(\%\left(\left[H^{+}, C^{-}\right] \succ B\right)\right)} P\left(B \succ\left[H^{+}, C^{-}\right]\right)^{(n)\left(\%\left(B \succ\left[H^{+}, C^{-}\right]\right)\right)} \times \\
& \prod P\left(\left[L^{+}, C^{-}\right] \succ B\right)^{(n)\left(\%\left(\left[L^{+}, C^{-}\right] \succ B\right)\right)} P\left(B \succ\left[L^{+}, C^{-}\right]\right)^{(n)\left(\%\left(B \succ\left[L^{+}, C^{-}\right]\right)\right)},
\end{aligned}
$$

where $n=102$ and $\%\left(H^{+} \succ A^{+}\right)$is the percentage of participants who preferred $H^{+}$to $A^{+}$, etc.

Unlike the analysis in Section EC.4, where we fit the choice data using prospect theory, we use a nonparametric procedure in which the utilities of the prospects are free parameters. Under this assumption, fitting the choice percentages in Table 2 of $\mathrm{Wu}$ and Markle (2007) involves estimating 6 free parameters for each of the 8 tests: $U\left(H^{+}\right), U\left(L^{+}\right), U\left(\left[H^{+}, C^{-}\right]\right), U\left(\left[L^{+}, C^{-}\right]\right), U\left(A^{+}\right)$, and $U(B)$ (since $U(\cdot)$ can be rescaled multiplicatively, we are free to set $\mu=1$ ). However, gain-loss separability imposes the restriction that $U\left(\left[H^{+}, C^{-}\right]\right)=U\left(H^{+}\right)+U\left(C^{-}\right)$and $U\left(\left[L^{+}, C^{-}\right]\right)=U\left(L^{+}\right)+U\left(C^{-}\right)$, leaving 5 free parameters: $U\left(H^{+}\right), U\left(L^{+}\right), U\left(C^{-}\right), U\left(A^{+}\right)$, and $U(B)$.

Thus, under both models,

$$
P\left(H^{+} \succ A^{+}\right)=\frac{1}{1+\exp \left(-\left[U\left(H^{+}\right)-U\left(A^{+}\right)\right]\right)}
$$

and

$$
P\left(L^{+} \succ A^{+}\right)=\frac{1}{1+\exp \left(-\left[U\left(L^{+}\right)-U\left(A^{+}\right)\right]\right)}
$$

However,

$$
P\left(\left[H^{+}, C^{-}\right] \succ B\right)=\frac{1}{1+\exp \left(-\left[U\left(H^{+}\right)+U\left(C^{-}\right)-U(B)\right]\right)}
$$

and

$$
P\left(\left[L^{+}, C^{-}\right] \succ B\right)=\frac{1}{1+\exp \left(-\left[U\left(L^{+}\right)+U\left(C^{-}\right)-U(B)\right]\right)},
$$

if we assume gain-loss separability and

$$
P\left(\left[H^{+}, C^{-}\right] \succ B\right)=\frac{1}{1+\exp \left(-\left[U\left(\left[H^{+}, C^{-}\right]\right)-U(B)\right]\right)}
$$

and

$$
P\left(\left[L^{+}, C^{-}\right] \succ B\right)=\frac{1}{1+\exp \left(-\left[U\left(\left[L^{+}, C^{-}\right]\right)-U(B)\right]\right)},
$$

if gain-loss separability is not assumed.

To test whether gain-loss separability is violated, we examine whether the unconstrained 6 parameter model in which gain-loss separability does not necessarily hold provides a significantly better fit to the data than the 5 parameter model that imposes gain-loss separability. Let $L_{0}\left(D ; U\left(H^{+}\right), U\left(L^{+}\right), U\left(C^{-}\right), U\left(A^{+}\right), U(B)\right)$ be the likelihood of the data assuming gain-loss separability, with $L_{0}^{*}(D)$ being the maximum likelihood for this model. Similarly, let $\left.L_{U}\left(D ; U\left(H^{+}\right), U\left(L^{+}\right), U\left(\left[H^{+}, C^{-}\right]\right), U\left(L^{+}, C^{-}\right]\right), U\left(A^{+}\right), U(B)\right)$ be the likelihood of the data if gainloss separability is relaxed, with $L_{U}^{*}(D)$ being the maximum likelihood for this model. Recall that 


Table EC.8
estimates (Tversky and Kahneman (1992) function),
single-domain gambles and mixed gambles (Study 2 )
\begin{tabular}{|c|ccccccc|}
\hline \multicolumn{8}{c|}{ Estimates for single-domain gambles $\left(\gamma_{S}\right)$} \\
$\alpha \backslash \lambda$ & 1.00 & 1.25 & 1.50 & 1.75 & 2.00 & 2.25 & 2.50 \\
\hline 0.4 & 0.876 & 0.812 & 0.745 & 0.736 & 0.695 & 0.586 & 0.428 \\
0.5 & 0.894 & 0.830 & 0.767 & 0.754 & 0.677 & 0.415 & 0.415 \\
0.6 & 0.912 & 0.812 & 0.803 & 0.740 & 0.654 & 0.437 & 0.437 \\
0.7 & 0.925 & 0.812 & 0.767 & 0.722 & 0.469 & 0.476 & 0.383 \\
0.8 & 0.383 & 0.383 & 0.383 & 0.383 & 0.383 & 0.383 & 0.383 \\
0.9 & 0.383 & 0.383 & 0.383 & 0.383 & 0.383 & 0.383 & 0.281 \\
1.0 & 0.383 & 0.383 & 0.383 & 0.281 & 0.281 & 0.281 & 0.270 \\
\hline
\end{tabular}

\begin{tabular}{|r|ccccccc|}
\hline & \multicolumn{7}{|c|}{ Estimates for mixed gambles $\left(\gamma_{M}\right)$} \\
$\alpha \backslash \lambda$ & 1.00 & 1.25 & 1.50 & 1.75 & 2.00 & 2.25 & 2.50 \\
\hline 0.4 & 0.491 & 0.507 & 0.514 & 0.517 & 0.499 & 0.433 & 0.270 \\
0.5 & 0.480 & 0.501 & 0.504 & 0.525 & 0.520 & 0.270 & 0.270 \\
0.6 & 0.471 & 0.494 & 0.512 & 0.521 & 0.507 & 0.270 & 0.270 \\
0.7 & 0.477 & 0.495 & 0.504 & 0.520 & 0.270 & 0.270 & 0.270 \\
0.8 & 0.390 & 0.390 & 0.370 & 0.270 & 0.270 & 0.270 & 0.270 \\
0.9 & 0.420 & 0.420 & 0.395 & 0.270 & 0.270 & 0.270 & 1.281 \\
1.0 & 0.470 & 0.495 & 0.520 & 1.281 & 1.281 & 1.281 & 1.270 \\
\hline
\end{tabular}

Table shows estimates for combinations of $\alpha$ and $\lambda$. For $\alpha \leq .7, \gamma_{S}$ is significantly larger than $\gamma_{M}$.

the statistic $2 \ln \left[L_{U}^{*}(D) / L_{0}^{*}(D)\right]$ is distributed approximately $\chi^{2}(1)$. The $p$-values for this test statistic for each of the 8 tests is shown in Table 2 of $\mathrm{Wu}$ and Markle (2007).

We also perform a test where we fit all 24 choices for the 8 tests. This test involves 20 free parameters for the model in which gain-loss separability is assumed (4 each for $U\left(H^{+}\right), U\left(L^{+}\right)$, and $U\left(C^{-}\right)$, and 8 for $U\left(A^{+}\right)$). The unconstrained model has 24 free parameters (4 each for $U\left(H^{+}\right)$, $U\left(L^{+}\right), U\left(\left[H^{+}, C^{-}\right]\right)$, and $U\left(\left[L^{+}, C^{-}\right]\right)$, and 8 for $\left.U\left(A^{+}\right)\right)$. Thus, the appropriate test statistic, $2 \ln \left[L_{U}^{*}(D) / L_{0}^{*}(D)\right]$ is distributed approximately $\chi^{2}(4)$. The results of this test are shown in the last row of Table 2 of $\mathrm{Wu}$ and Markle (2007).

\section{EC.7. Weighting Function Estimation for Study 2}

We repeated the same analysis from Section EC.5 for the data from Study 2. Each of the 102 participants in Study 2 made 24 choices, thus the likelihood is maximized over the 2448 choices in Table 2 of $\mathrm{Wu}$ and Markle (2007). All other aspects of the procedure were identical.

As with Study 1, our base analysis assumed that $\alpha=0.5$ and $\lambda=2$. The resulting parameter estimates were very close to the Study 1 estimates: $\hat{\gamma}_{S}=.68$ and $\hat{\gamma}_{M}=.52$, a difference that was statistically significant $(t=8.28, p<.0001)$. The best-fitting scaling parameter was $\hat{\mu}=.28$, somewhat higher than the estimate of Study 1. The estimates for the Prelec weighting function were also remarkably close to those found for the Study 1 data: $\hat{\beta}_{S}=.64$ and $\hat{\beta}_{M}=.44(p<.0001)$.

We also conducted sensitivity analyses on $\alpha$ and $\lambda$ to test whether these differences were robust. Table EC. 8 shows that $\hat{\gamma}_{S}>\hat{\gamma}_{M}$ for most values of $\alpha$ and $\lambda$. However, unlike Study $1, \hat{\gamma}_{S}<\hat{\gamma}_{M}$ when $\alpha \geq .9$. It is important to note, however, that the fits for $\alpha \geq .9$ were notably worse than the fits when $\alpha$ was low. In particular, $\hat{\alpha}=.54$ and $\hat{\lambda}=1.68$ when we allowed $\alpha$ and $\lambda$ to be free parameters.

\section{EC.8. Summary}

We have presented a number of error models, including models in which error is random and unsystematic and models in which error is systematic. We derived implications of these error models 
on choice percentages, violations of double matching, conditional probabilities, and error rates. The double matching data of Study 1 of Wu and Markle (2007) are better explained by the asymmetric error model than either the null or mixed error models.

We also details of a procedure in which we estimated the probability weighting function for single-domain gambles and mixed gambles. We found that the probability weighting function for mixed gambles was considerably more curved than the weighting function single-domain gambles for the data for both Studies 1 and 2 .

\section{References}

See references list in the main paper. 